Universidade de Brasília

Instituto de Ciências Exatas

Departamento de Matemática

\title{
Transformações de Bäcklund no Espaço-Tempo de Minkowski 3-dimensional
}

\author{
por
}

Filipe Kelmer Alves

Orientadora: Prof ${ }^{\mathrm{a}}$. Dr ${ }^{\mathrm{a}}$. Luciana Maria Dias de Ávila Rodrigues

Brasília

2016 


\section{Transformações de Bäcklund no Espaço-Tempo de Minkowski 3-dimensional por}

\section{Filipe Kelmer Alves *}

Dissertação apresentada ao Departamento de Matemática da Universidade de Brasília, como parte dos requisitos para obtenção do grau de

\section{MESTRE EM MATEMÁTICA}

Brasília, 16 de fevereiro de 2017.

Comissão Examinadora:

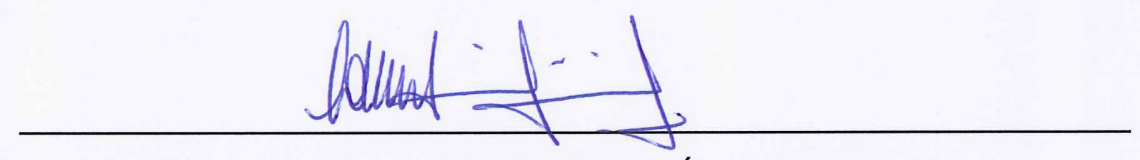

Profa. Dra. Luciana Maria Dias de Ávila Rodrigues

MAT/UnB (Orientadora)
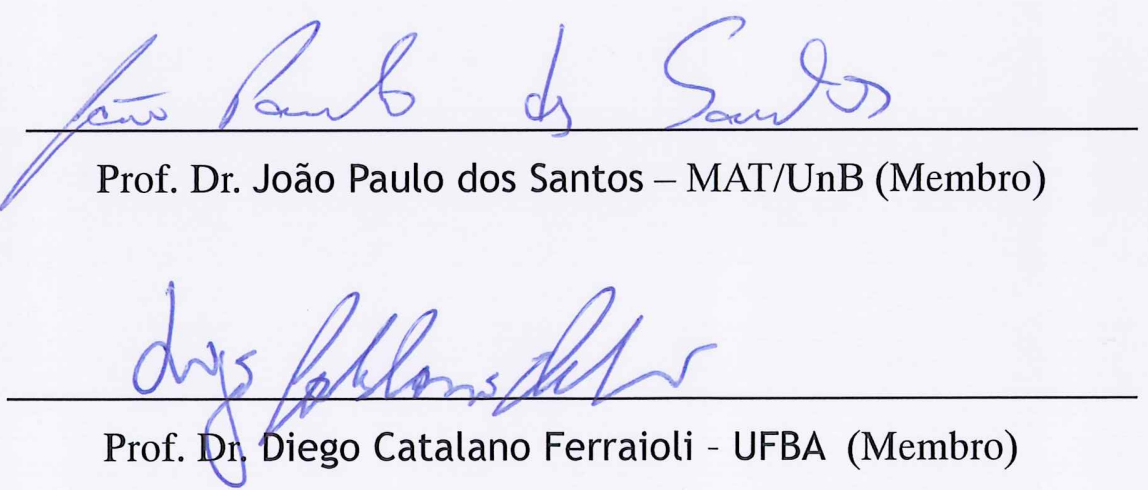

* O autor foi bolsista do CNPq durante a elaboração desta dissertação. 
Ficha catalográfica elaborada automaticamente, com os dados fornecidos pelo(a) autor(a)

Transformações de Bäcklund no Espaço-Tempo de Minkowski 3-Dimensional / Filipe Kelmer Alves; orientador Luciana Maria Dias de Ávila Rodrigues. -Brasilia, 2017. $128 \mathrm{p}$.

Dissertação (Mestrado - Mestrado em Matemática) -Universidade de Brasília, 2017.

1. Espaço-Tempo de Minkowski. 2. Transformação de Bäcklund. 3. Teorema de Bäcklund. 4. Equação de sine Gordon. 5. Congruência Pseudo-Esférica. I. Maria Dias de Ávila Rodrigues, Luciana, orient. II. Título. 
Aos meus Pais Geraldo e Simone,

à minha irmã Carol e a minha adorável Maria Eugênia. 


\section{Agradecimentos}

Agradeço principalmente ao meu pai Geraldo e minha mãe Simone. Todas minhas conquistas acadêmicas devem ser creditadas a eles.

À toda minha família, Carol, Maria Eugênia, avôs, padrinhos, tios e primos.

Aos meus amigos.

À professora Luciana.

A todos do programa de Pós-Graduação e ao CNPq. 
"For those about to rock, we salute you" $\mathrm{AC} / \mathrm{DC}$ 


\section{Resumo}

Nesta dissertação discutiremos sobre as Transformações de Bäcklund entre superfícies no espaço-tempo de Minkowski. Apresentaremos a versão clássica da Transformação de Bäcklund no Espaço Euclidiano e vamos generalizá-la para o Espaço de Minkowski e analisar suas propriedades. Mostraremos que existem análogos, no Espaço de Minkowski do Teorema de Bäcklund, Teorema de Integrabilidade, relações entre superfícies e soluções de equações diferenciais parciais e existência de família das soluções, que foram obtidas por [15], 4], 10], [11, [19] e [12].

Palavras-chave: Espaço-Tempo de Minkowski, Transformação de Bäcklund, Teorema de Bäcklund, Equação de sine-Gordon, Congruência Pseudo-Esférica. 


\section{Abstract}

In this work we shall discuss about Bäcklund's Transformation in Minkowski SpaceTime. Our main goal is to establish, in Minkowski Space, the Euclidian classical results such as Bäcklund's Theorem, Integrability Conditions and their relation between existence of surfaces related to Partial Differential Equations solutions, each one can be found in following works [15], [4], [10], [11], [19] and [12].

Keywords: Minkowski Space-Time, Bäcklund's Transformation, Pseudo-Spherical Congruence, Bäcklund Transformation, Sine-Gordon equation. 


\section{Sumário}

$\begin{array}{ll}\text { Introdução } & 1\end{array}$

$\begin{array}{lll}1 & \text { Geometria diferencial no espaço de Minkowski } & 5\end{array}$

1.1 O Espaço de Minkowski . . . . . . . . . . . . . . . . . . . . 5

1.1 .1 Definições básicas $\ldots \ldots \ldots \ldots \ldots \ldots$

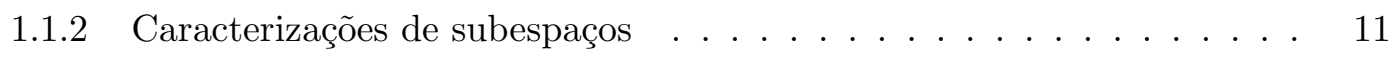

$1.1 .3 \quad$ Propriedades geométricas dos vetores em $\mathbb{L}^{3} \ldots \ldots$. . . . . . . . 15

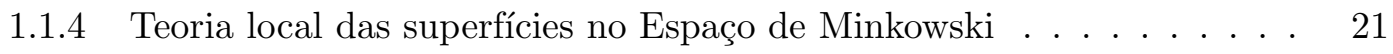

2 Formas Diferenciais e o Método do Referencial Móvel 35

2.1 Formas Diferenciais . . . . . . . . . . . . . . . . . . . . . . 35

2.2 Método do Referencial Móvel em $\mathbb{L}^{3} \ldots \ldots \ldots \ldots$. . . . . . . . . . . 45

$2.2 .1 \quad$ Referenciais Móveis em $\mathbb{E}^{3} \ldots \ldots \ldots$. . . . . . . . . 46

2.2 .2 Referenciais Móveis em $\mathbb{L}^{3} \ldots \ldots \ldots \ldots$. . . . . . . . . 54

$2.2 .3 \quad$ Referenciais Nulos em $\mathbb{L}^{3} \ldots \ldots \ldots \ldots$. . . . . . . . . . 62

3 Superfícies não-degeneradas de curvatura constante não nula em $\mathbb{L}^{3}$ e suas

$\begin{array}{lc}\text { EDP's. } & 68\end{array}$

$3.1 \quad$ Superfícies de curvatura Gaussiana constante não nula em $\mathbb{E}^{3}$. . . . . . . . . 70

3.1.1 Curvatura constante negativa em $\mathbb{E}^{3} \ldots \ldots \ldots$. . . . . . . . 70

$3.1 .2 \quad$ Curvatura constante positiva em $\mathbb{E}^{3} \ldots \ldots \ldots \ldots$

$3.2 \quad$ Superfícies de curvatura constante não nula em $\mathbb{L}^{3} \ldots \ldots \ldots$. . . . . . . . 77

3.2.1 $\quad$ Superfícies Weingarten-diagonalizáveis . . . . . . . . . . . . . . . 77

3.2.2 Superfícies não Weingarten-diagonalizáveis . . . . . . . . . . . . . 84

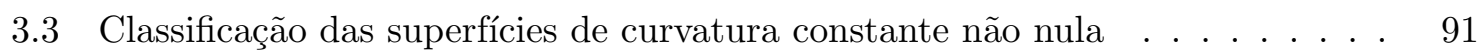


$\begin{array}{|ll|}4 & \text { Transformações de Bäcklund em } \mathbb{L}^{3}\end{array}$

4.1 Congruência Pseudo-Esférica no espaço Euclidiano . . . . . . . . . . . . . . 95

$4.2 \quad$ Congruência Pseudo-Esférica Generalizada $\ldots \ldots \ldots \ldots$. . . . . . . . . . . . . 99

4.3 Condições de Integrabilidade $\ldots \ldots \ldots$. . . . . . . . . . . . . . . 107

4.3 .1 Caso Clássico . . . . . . . . . . . . . . . . . . . . . . . . 110

4.3 .2 Caso Generalizado . . . . . . . . . . . . . . . . . . 115

$\begin{array}{lll}5 \text { Conclusões } & 120\end{array}$ 


\section{Introdução}

Nesta dissertação vamos discutir sobre a adaptação do Teorema de Bäcklund para EspaçoTempo de Minkowski e algumas de suas consequências.

Sabe-se, desde o século XIX, que a cada solução da Equação de sine-Gordon

$$
\alpha_{x x}-\alpha_{y y}=\sin \alpha
$$

faz-se corresponder a uma superfície em $\mathbb{R}^{3}$ com curvatura Gaussiana constante negativa. Em 1875 [1] o Físico-Matemático Albert Victor Bäcklund mostrou a existência de uma transformação entre superfícies com curvatura Gaussiana negativa.

Usando tal transformação, Bäcklund mostrou como obter uma hierarquia de soluções da equação de sine-Gordon a partir de uma solução inicial. Esta técnica ganhou destaque no século passado devido a importância que a Equação de sine-Gordon tem em vários ramos da ciência, como em Física-Matemática, Sistemas Dinâmicos Integráveis, Teoria dos Sólitons e etc. De modo que esta transformação, conhecida como Transformação de Bäcklund, se tornou um tema clássico.

A partir de 1905, com o surgimento da Teoria da Relatividade Restrita, o Espaço-Tempo de Minkowski ficou em evidência. O que motivou a investigação da Geometria Diferencial neste espaço, bem como o estudo de Superfícies no Espaço-Tempo de Minkowski.

Neste contexto, surge a idéia de investigar se existe um análogo a Transformação de Bäcklund para superfícies no Espaço-Tempo de Minkowski e ainda se tal transformação permite uma relação entre soluções de alguma equação diferencial.

O Espaço-Tempo de Minkowski 3-dimensional é o conjunto $\mathbb{R}^{3}$ munido da Métrica de Lorentz

$$
\left\langle\left(x_{1}, x_{2}, x_{3}\right),\left(y_{1}, y_{2}, y_{3}\right)\right\rangle=x_{1} y_{1}+x_{2} y_{2}-x_{3} y_{3} .
$$

Esta métrica não é necessariamente positiva definida e difere da Métrica Euclidiana, que é positiva definida, por um sinal no último termo. As superfícies no Espaço-Tempo de Min- 
kowski são ditas Tipo-Tempo, Tipo-Espaço ou Tipo-Luz dependendo da métrica induzida na superfície ser indefinida e não-degenerada, positiva definida ou degenerada respectivamente.

Em 1980 Louise V. McNertney em sua Tese de Doutorado [15] "One-parameter families of surfaces with constant curvature in Lorentz 3-space", mostrou que existia um análogo da Transformação de Bäcklund para Superfícies Tipo-Tempo com curvatura constante negativa ou positiva. Mostrou ainda que tais superfícies correspondiam as equações de sinh-Gordon $\left(\alpha_{x x}-\alpha_{y y}=\sinh \alpha\right)$ e Liouville $\left(\alpha_{u v}=-\frac{1}{2} e^{-\alpha}\right)$. Consequentemente McNertney mostrou como construir uma hierarquia de soluções destas duas equações usando a Transformação de Bäcklund.

Em 1981 S. S. Chern no Artigo [4] "Geometrical Interpretation of the Sinh-Gordon Equation" mostrou uma técnica para corresponder Superfícies Tipo-Espaço ou Tipo-Tempo com curvatura Gaussiana constante com as equações de Sin-Gordon e Sinh-Gordon. Do mesmo modo, em 1985, H. Hesheng [10] no Artigo "The Constrution of Hyperbolic Surfaces in 3 Dimensional Minkowski Space and Sinh-Laplace Equation"aplicou a técnica de Chern e obteve superfícies no Espaço-Tempo de Minkowski que correspondiam com soluções da equação de sine-Laplace $\left(\alpha_{x x}+\alpha_{y y}=\sin \alpha\right)$ e Sinh-Laplace $\left(\alpha_{x x}+\alpha_{y y}=\sinh \alpha\right)$.

Em 1990 Bennett Palmer [19] no Artigo "Bäcklund transformations for surfaces in Minkowski space" mostrou como obter uma Transformação de Bäcklund que transforma uma superfície Tipo-Espaço com curvatura Gaussiana negativa em outra superfície Tipo-Tempo com curvatura Gaussiana negativa. Consequentemente mostrou como corresponder soluções da equação de Sin-Laplace com soluções da Sinh-Laplace.

Em 1997 C. Tian [21], no Artigo "Bäcklund transformation on surfaces with $K=-1$ in $\mathbb{R}^{2,1}$ ", descreveu a Transformação de Bäcklund entre superfícies Tipo-Espaço com curvatura constante negativa.

Por último, em 2002 C. H. Gu, H. S. Hu e J. Inoguchi [12] no Artigo "On time-like surfaces of positive constant gaussian curvature and imaginary principals curvatures" mostraram como obter a Transformação de Bäcklund para superfícies Tipo-Tempo cujas curvaturas principais são imaginárias. E ainda mostraram que, com tal Transformação, é possível obter uma hierarquia de soluções da equação de cosh-Gordon $\left(\alpha_{x x}-\alpha_{y y}=\cosh \alpha\right)$.

Nesta dissertação, baseada nas seis últimas referências citadas acima, vamos mostrar como as superfícies não-degeneradas com curvatura Gaussiana constante não nulas estão relacionadas com soluções de certas Equações diferenciais parciais, ditas EDP's Naturais. Veremos que estas superfícies podem ser classificadas por, ao todo, seis EDP's Naturais. 
Mostraremos como a Transformação de Bäcklund pode ser generalizada para superfícies Não Degeneradas no Espaço-Tempo. E também, que estas Transformações ocorrem somente entre superfícies não-degeneradas com curvatura constante não nula quais correspondem exatamente com as superfícies classificadas via EDP's Naturais.

Veremos que existem ao todo oito tipos de Transformações de Bäcklund e mostraremos como obter cada uma delas.

Neste texto temos também como objetivo, comparar os resultados obtidos entre o Espaço Euclidiano e o Espaço-Tempo de Minkowski, de modo que todas as definições e resultados serão dados paralelamente para os dois casos, de modo que fique evidente as diferenças e semelhanças em ambos os contextos.

Esperamos que este texto seja auto-contido, no sentido que as demonstrações feitas dependam somente de resultados básicos ou de resultados que tenhamos mostrado anteriormente. Para isso incluímos os dois primeiros Capítulos nos quais introduziremos a Geometria Diferencial no Espaço-Tempo de Minkowski e o conceito de Referencial Móvel. Deste modo os pré-requisitos à leitura deste texto são conceitos de Álgebra Linear e alguma familiaridade com o conceito de Superfícies em Geometria Diferencial.

No Capítulo 1 introduzimos a Geometria Diferencial no Espaço-Tempo de Minkowski. Começamos descrevendo como se comporta a Métrica de Lorentz e suas principais propriedades. Em seguida trataremos de vetores e suas propriedades geométricas. Por último traremos o conceito de Superfícies.

A principal ferramenta utilizada nos Artigos citados acima é o Método do Referencial Móvel, que foi desenvolvido no século passado pelo matemático francês Élie Joseph Cartan. Introduziremos este método no Capítulo 2 no qual traremos o conceito de Formas Diferenciais e a noção de Referencial Móvel no Espaço Euclidiano. Em sequência adaptamos o conceito de Referencial Móvel para Superfícies no Espaço-Tempo de Minkowski.

A primeira aplicação do Método do Referencial Móvel aparece no Capítulo3. Começaremos mostrando como pode ser obtida a famosa relação entre as Superfícies de curvatura Gaussina constante negativa no Espaço Euclidiano com soluções da sua EDP natural: a Equação de sine-Gordon. Em seguida mostraremos como obter a EDP natural de cada superfície nãodegenerada de curvatura Gaussiana constante no Espaço-Tempo de Minkowski. Mais precisamente, mostraremos que cada superfície não-degenerada com curvatura constante não nula pode ser correspondida a uma solução de uma certa EDP, e reciprocamente a cada solução desta EDP existirá uma superfície não-degenerada de curvatura Gaussiana constante. 
Por último, no Capítulo 4, mostraremos como a Transformação de Bäcklund pode ser obtida através do conceito de Congruência Pseudo-Esférica, tanto para os casos Euclidianos quanto para os casos no Espaço de Minkowski. Em seguida demonstraremos o famoso Teorema de Bäcklund no espaço Euclidiano e sua generalização para o Espaço-Tempo de Minkowski que garante que a Transformação de Bäcklund pode ocorrer somente entre as superfícies classificadas no Capítulo 3. Por último demonstraremos os Teoremas de Integrabilidade, isto é resultados que nos garantem a existência de Congruências Pseudo-Esféricas e consequentemente de Transformações de Bäcklund. 


\section{Capítulo 1}

\section{Geometria diferencial no espaço de}

\section{Minkowski}

\section{$1.1 \quad$ O Espaço de Minkowski}

\subsubsection{Definições básicas}

Nesta seção começaremos com algumas definições e resultados de Álgebra Linear a respeito das formas bilineares. Em seguida introduziremos os conceitos básicos relacionados ao Espaço de Minkowski, bem como sua definição e suas propriedades básicas.

Seja $V$ um espaço vetorial sobre o corpo dos números reais com dimensão finita $n$. Comecemos com o conceito de forma bilinear sobre um espaço vetorial;

Definição 1.1. Dizemos que a aplicação $b: V \times V \rightarrow \mathbb{R}$ é uma forma bilinear sobre $V$ desde que, a cada $v \in V$ verifica-se que

$$
\begin{aligned}
& b(v, \bullet): V \rightarrow \mathbb{R} ; w \in V \mapsto b(v, w), \\
& b(\bullet, v): V \rightarrow \mathbb{R} ; \quad w \in V \mapsto b(w, v),
\end{aligned}
$$

são aplicações lineares em $V$. Além disso, dizemos que b é uma forma bilinear simétrica desde que para cada $v, w \in V$ verifica-se que $b(v, w)=b(w, v)$.

Dada uma matriz $C=\left(c_{i j}\right), n \times n$, com coeficientes no corpo $\mathbb{R}$ podemos fazê-la corresponder a uma forma bilinear sobre $\mathbb{R}^{n}$ da seguinte maneira: se $x=\left(x_{1}, \ldots, x_{n}\right)$ e $y=\left(y_{1}, \ldots, y_{n}\right)$ 
são vetores em $\mathbb{R}^{n}$ denotados por matrizes colunas $n \times 1$, então

$$
(x, y) \mapsto x^{t} C y=\left(x_{1}, \ldots, x_{n}\right)\left(\begin{array}{ccc}
c_{11} & \ldots & c_{1 n} \\
& \ldots & \\
c_{n 1} & \ldots & c_{n n}
\end{array}\right)\left(\begin{array}{c}
y_{1} \\
\ldots \\
y_{n}
\end{array}\right) \in \mathbb{R}
$$

em que o subscrito $t$ denota a transposta da matriz. A bilinearidade de tal forma decorrerá da linearidade da multiplicação de matrizes.

Proposição 1.1. Uma matriz $C=\left(c_{i j}\right)$ em $\mathbb{R}$ representa uma forma bilinear simétrica sobre $\mathbb{R}^{n}$ se, e somente se, ela for uma matriz simétrica.

Demonstração: Suponhamos que $C$ seja uma matriz simétrica, isto é, $C^{t}=C$. Da Equação 1.1., $x^{t} C y$ é um número real assim, $\left.\left(x^{t} C y\right)^{t}=\left(x^{t} C y\right)\right)$. Por outro lado, como $C$ é simétrica

$$
\left(x^{t} C y\right)^{t}=y^{t} C^{t} x=y^{t} C x
$$

donde $x^{t} C y=y^{t} C x$ e a forma bilinear induzida por $C$ é simétrica.

Reciprocamente, suponhamos que $C$ representa uma forma bilinear simétrica em $\mathbb{R}^{n}$, isto é, para todo $x, y \in \mathbb{R}^{n}$ verifica-se $x^{t} C y=y^{t} C x$. Como

$$
y^{t} C x=\left(y^{t} C x\right)^{t}=x^{t} C^{t} y=y^{t} C^{t} x,
$$

temos $y^{t}\left(C-C^{t}\right) x=0$ para todo $x, y$. Donde $C=C^{t}$ e segue que $C$ é uma matriz simétrica.

Vimos que matrizes $n \times n$ com coeficientes no corpo $\mathbb{R}$ correspondem a uma forma bilinear em $\mathbb{R}^{n}$. Vamos mostrar agora que cada forma forma bilinear em um espaço vetorial $V$ corresponde a uma forma bilinear em $\mathbb{R}^{n}$. Seja $\left\{\alpha_{1}, \ldots \alpha_{n}\right\}$ uma base qualquer de $V$ e sejam $v, w$ elementos de $V$ que podem ser expressos em termos desta base por

$$
\begin{array}{r}
v=v_{1} \alpha_{1}+\ldots+v_{n} \alpha_{n} \\
w=w_{1} \alpha_{1}+\ldots+w_{n} \alpha_{n} .
\end{array}
$$

Pela bilinearidade de $b$,

$$
b(v, w)=\sum_{i, j=1}^{n} x_{i} y_{j} b\left(\alpha_{i}, \alpha_{j}\right) .
$$

Denotando a matriz $\left(b_{i j}\right)=\left(b\left(\alpha_{i}, \alpha_{j}\right)\right)$ temos que $\left(b_{i j}\right)$ representa uma forma bilinear sobre $\mathbb{R}^{n}$. Deste modo fixado uma base para o espaço vetorial $V$ temos uma única representação 
matricial para as formas bilineares. Além disso, pela Proposição (1.1) a forma bilinear será simétrica se, e somente se, sua matriz representante for uma matriz simétrica.

Seja $b$ uma forma bilinear em $V$ e $B$ sua matriz representativa em uma dada base de $V$. Suponhamos que mudamos a base do espaço vetorial $V$ para uma nova base. Denote por $N$ a matriz não singular de passagem (ou matriz de mudança de bases) da base nova para base antiga, de modo que $v=N v^{\prime}$ e $w=N w^{\prime}$ em que $v^{\prime}$ e $w^{\prime}$ são as coordenadas de $v$ e $w$ nesta nova base. Nestas condições, obtemos que

$$
v^{t} B w=\left(N v^{\prime}\right)^{t} B\left(N w^{\prime}\right)=\left(v^{\prime}\right)^{t}\left(N^{t} B N\right) w^{\prime} .
$$

Assim a matriz representativa de forma bilinear $b$ nesta nova base é dada por;

$$
N^{t} B N \text {. }
$$

Observe que as matrizes representantes das transformações lineares se alteram em novas bases em termos da inversa da matriz de passagem, ao passo que as matrizes representantes de formas bilineares se alteram em termos da transposta.

Definição 1.2. Seja b uma forma bilinear simétrica sobre $V$ espaço vetorial real de dimensão finita. Chamamos de forma quadrática determinada por $b$ a função

$$
f: V \rightarrow \mathbb{R} ; \quad v \mapsto f(v)=b(v, v)
$$

Em termos da matriz representante de $b$, uma forma quadrática $f$ é expressa por $f(v)=$ $\sum_{i=1}^{n} x_{i}^{2} b_{i j}$. Além disso, conhecendo a forma quadrática $f$, a identidade

$$
b(v, w)=\frac{1}{2}(b(v+w, v+w)-b(v, v)-b(w, w))=\frac{1}{2}(f(v+w)-f(v)-f(w)),
$$

nos permite construir a forma bilinear simétrica $b$.

Definição 1.3. Seja $V$ um espaço vetorial real de dimensão finita e b uma forma bilinear simétrica em $V$. Dizemos que:

1. b é positiva definida (respectivamente negativa definida) desde que, dado $v \neq 0 \mathrm{em}$ $V$ tivermos $b(v, v)>0$ (respectivamente $<0)$;

2. b é não-degenerada, desde que, para cada $v \in V$ tal que $b(v, w)=0$ para todo $w \in V$ tivermos $v=0$.

Similarmente, dizemos que b é indefinida caso não seja positiva definida nem negativa definida e também será degenerada caso não se verifique o segundo item. 
Dado qualquer subespaço vetorial $W$ de $V$ fica definida uma aplicação $\left.b\right|_{W}$ em $W \times W$ dada pela restrição de $b$ a $W \times W$. Além disso, $\left.b\right|_{W}$ será uma forma bilinear em $W$ que será também simétrica caso $b$ o seja. Além disso se $b$ é positiva ou negativa definida também verificaremos que $\left.b\right|_{W}$ o é. Porém note que se $b$ é degenerada nada podemos concluir sobre $\left.b\right|_{W}$.

Proposição 1.2. Seja b uma forma bilinear simétrica em $V$ espaço vetorial real de dimensão finita $n$. Então a forma b é não-degenerada se, e somente se, sua matriz representante em alguma (logo em qualquer) base é uma matriz inversível.

Demonstração: Seja $B=\left(b_{i j}\right)$ a matriz representante da forma $b$ em uma base $\left\{\alpha_{1}, \ldots, \alpha_{n}\right\}$ de $V$ e denote por $b^{i}=\left(b_{1 i}, b_{2 i}, \ldots, b_{n i}\right)$, com $i \in\{1,2, \ldots, n\}$, a $i$-ésima coluna de $B$. Lembramos que a matriz $B$ é inversível se, e somente se, suas colunas são linearmente independentes. Por bilinearidade $b$ verificamos que $b(v, w)=0$ para todo $w \in V$ se, e somente se, $b\left(v, \alpha_{i}\right)=0$ para cada $\alpha_{i}$. Deste modo $b$ é degenerada se, e somente se, existe $v=v_{1} \alpha_{1}, \ldots, v_{n} \alpha_{n} \neq 0$ (isto é, pelo menos um dos $v_{i}$ é não nulo) tal que, para cada $i$,

$$
0=b\left(v, \alpha_{i}\right)=\sum_{j=1}^{n} v_{j} b\left(\alpha_{j}, \alpha_{i}\right)=\sum_{j=1}^{n} v_{j} b_{j i}
$$

ou ainda

$$
0=v_{1} b^{1}+v_{2} b^{2}+\ldots+v_{n} b^{n}
$$

o que equivale a dizer que as colunas de $B$ são lineramente dependentes. Deste modo garantimos que $b$ é não-degenerada se, e somente se, sua matriz representativa em alguma base é invertível.

Definição 1.4. Seja $b$ uma forma bilinear simétrica em $V$. Dizemos que o número inteiro $\nu$ é o indice da forma $b \mathrm{em} V$, desde que seja o maior número inteiro que coincide com a dimensão de algum sub espaço vetorial $W$ de $V$ tal que $\left.b\right|_{W}$ é uma forma negativa definida em $W$.

Consequentemente, dado uma forma bilinear simétrica em $V$, seu índice $\nu$ em $V$ pertence ao conjunto $\{0,1, \ldots, \operatorname{dim}(V)\}$. Além disso, $b$ é positiva definida em $V$ se, e somente se, seu índice $\nu$ em $V$ é igual a 0 , e também será negativa definida em $V$ se, e somente se, seu índice em $V$ é igual a $\operatorname{dim}(V)$. 
Vimos que uma forma bilinear $b$ em um espaço vetorial $V$, se suposta simétrica, dá origem a uma forma quadrática $f$ em $V$. Supondo adicionalmente que $b$ seja não-degenerada temos a;

Definição 1.5. Seja $V$ um espaço vetorial real de dimensão finita. Então, dizemos que

$$
g: V \times V \rightarrow \mathbb{R} ; \quad(v, w) \mapsto g(v, w) \in \mathbb{R},
$$

é um produto interno em $V$ desde que, se uma forma bilinear simétrica não-degenerada em $V$. Denotamos também $g(v, w)$ por $\langle v, w\rangle$.

Se um espaço vetorial $V$ admite um produto interno $g$ podemos dar aos seus vetores uma noção de ortogonalidade. Dados dois vetores $v, w \in V$ dizemos que são ortogonais, e escrevemos que $v \perp w$, desde que, $g(v, w)=0$. Deste modo por bilinearidade de $g, 0 \perp v$ para qualquer $v \in V$. Além disso, temos também a noção de subconjuntos ortogonais, dizemos que $A, B \subset V$ são ortogonais, e escrevemos $A \perp B$, desde que, $a \perp b$ para cada $a \in A$ e $b \in B$. Dado um subconjunto $A$ de $V$ denotamos por $A^{\perp}$ o conjunto dos elementos ortogonais a $A$. Note que em geral, se um produto interno não é positivo definido podem existir vetores não nulos em $v \in V$ tais que $g(v, v)=0$ e isto nos diz que dado um subespaço vetorial $W$ de $V$ nem sempre se verifica que $V$ é dado pela soma direta de $W$ com $W^{\perp}$.

Além disso em espaços vetoriais com produto interno podemos falar de bases ortogonais;

Definição 1.6. Seja $V$ um espaço vetorial real de dimensão $n$ com um produto interno $g$. Dizemos que uma base $\left\{\alpha_{1}, \ldots, \alpha_{n}\right\}$ é uma base ortogonal desde que $g\left(\alpha_{i}, \alpha_{j}\right)=0$ para todo $i \neq j$.

Além disso é possível mostrar que qualquer espaço vetorial real de dimensão finita com um produto interno admite uma base ortogonal pelo processo de Grand-Shimidt.

Dada uma base ortogonal $\left\{\alpha_{1}, \ldots, \alpha_{n}\right\}$ de $V$ podemos supô-la ser ortonormal isto é, além de ortogonal se verifica para cada $i$ que $g\left(\alpha_{i}, \alpha_{i}\right) \in\{-1,0,1\}$, bastando trocar se necessário, $\alpha_{i}$ por

$$
\begin{array}{ll}
\frac{\alpha_{i}}{\sqrt{g\left(\alpha_{i}, \alpha_{i}\right)}}, & \text { se } g\left(\alpha_{i}, \alpha_{i}\right)>0 \\
\frac{\alpha_{i}}{\sqrt{-g\left(\alpha_{i}, \alpha_{i}\right)}}, & \text { se } g\left(\alpha_{i}, \alpha_{i}\right)<0
\end{array}
$$

A partir de agora nos restringiremos ao caso de interesse deste texto; o espaço vetorial real $\mathbb{R}^{3}$. 
Dado $\mathbb{R}^{3}$, considere sua estrutura usual de espaço vetorial sobre o corpo de escalares reais $\mathbb{R}$, de modo que, com a base canônica $B=\left\{e_{1}, e_{2}, e_{3}\right\}$, sendo $e_{1}=(1,0,0), e_{2}=(0,1,0)$ e $e_{3}=(0,0,1)$, um vetor $u \in \mathbb{R}^{3}$ pode ter suas coordenadas denotadas por $\left(u_{1}, u_{2}, u_{3}\right)$ em relação a base $B$. Neste contexto definimos o espaço de Lorentz-Minkowski;

Definição 1.7. Definimos o Espaço de Minkowisk como sendo um par $\mathbb{L}^{3}=\left(\mathbb{R}^{3},\langle\rangle,\right)$, como sendo o espaço vetorial $\mathbb{R}^{3}$ munido do produto interno $\langle\rangle:, \mathbb{R}^{3} \times \mathbb{R}^{3} \rightarrow \mathbb{R}$ definido pela regra que associa $u=\left(u_{1}, u_{2}, u_{3}\right)$ e $v=\left(v_{1}, v_{2}, v_{3}\right)$ pertencentes a $\mathbb{R}^{3}$ ao número real

$$
\langle u, v\rangle=u_{1} v_{1}+u_{2} v_{2}-u_{3} v_{3} \in \mathbb{R} .
$$

A forma $g=\langle$,$\rangle é uma métrica, chamada de métrica de Lorentz.$

Temos que a métrica $g$ como dada acima é, de fato, uma forma bilinear, simétrica, nãodegenerada com índice $\nu=1 \mathrm{em} \mathbb{R}^{3}$.

Sempre podemos considerar métrica euclidiana de $\mathbb{R}^{3}$, qual denotaremos por $\langle,\rangle_{\mathbb{E}^{3}}$, dada pela regra $\langle u, v\rangle_{\mathbb{E}^{3}}=u_{1} v_{1}+u_{2} v_{2}+u_{3} v_{3}$. De modo que os espaços métricos $\mathbb{E}^{3}=\left(\mathbb{R}^{3},\langle,\rangle_{\mathbb{E}^{3}}\right)$ $\mathrm{e} \mathbb{L}^{3}=\left(\mathbb{R}^{3},\langle\rangle,\right)$ são espaços métricos distintos, além do que, a métrica de Lorentz apresenta várias diferenças com a métrica cartesiana. Uma das principais diferenças é que a métrica de Lorentz não é positiva definida, isto é, não satisfaz a propriedade: $\forall u \in \mathbb{R}^{3}$, tem-se $\langle u, u\rangle \geq 0$, com ainda, a igualdade ocorrendo se, e somente se, $u=0$. De fato, basta considerar $u=e_{3}$, pois, $\left\langle e_{3}, e_{3}\right\rangle=-1<0$.

Deste modo temos a seguinte classificação para os vetores $u \in \mathbb{L}^{3}$;

Definição 1.8. Dado $u \in \mathbb{L}^{3}$ dizemos que u é um vetor

1. tipo-espaço, desde que, $\langle u, u\rangle>0$ ou $u=0$;

2. tipo-tempo, desde que, $\langle u, u\rangle<0$;

3. tipo-luz, desde que, $\langle u, u\rangle=0$ e $u \neq 0$.

Nos referiremos a esta classificação como caráter causal do vetor $u \in \mathbb{L}^{3}$.

Com esta nomenclatura temos que, por exemplo, $e_{1}$ é um vetor tipo-espaço, $e_{3}$ é um vetor tipo-tempo, $e_{2}+e_{3}$ é um vetor tipo-luz. E também, com esta definição caracterizamos todos os vetores do espaço de Minkowski pela união disjunta, $\mathbb{L}^{3}=\mathcal{E} \cup \mathcal{T} \cup \mathcal{L}$, onde $\mathcal{E}=\left\{u \in \mathbb{L}^{3} \mid u\right.$ é vetor tipo-espaço $\}, \mathcal{T}=\left\{u \in \mathbb{L}^{3} \mid u\right.$ é vetor tipo-tempo $\}$ e $\mathcal{L}=\{u \in$ $\mathbb{L}^{3} \mid u$ é vetor tipo-luz\}. 


\subsubsection{Caracterizações de subespaços}

Dado um subespaço vetorial $U$ do espaço vetorial $\mathbb{L}^{3}$, podemos sempre introduzir a métrica de Lorentz em $U$ simplesmente tomando a restrição de $\langle$,$\rangle aos vetores de U$. Assim considerando $U \subset \mathbb{L}^{3}$ como espaço métrico, com a métrica induzida, temos a seguinte classificação causal para todos os possíveis subespaços de $\mathbb{L}^{3}$ :

Definição 1.9. Dado $U \subset \mathbb{L}^{3}$ subespaço vetorial, dizemos que $U$ é:

1. tipo-espaço, desde que sua métrica induzida seja positiva definida ou se $U=0$;

2. tipo-tempo, desde que sua métrica induzida seja não-degenerada de índice 1;

3. tipo-luz, desde que sua métrica induzida seja degenerada e $U \neq\{0\}$.

Dado um subconjunto finito $A=\left\{u_{1}, \ldots, u_{n}\right\} \subset \mathbb{R}^{3}$, denotamos por ger $\left\{u_{1}, \ldots, u_{n}\right\}=$ $\left\langle\left\{u_{1}, \ldots, u_{n}\right\}\right\rangle$ o subespaço vetorial gerado por todas as combinações lineares dos elementos de $A$ por escalares reais, isto é, $\left\langle\left\{u_{1}, \ldots, u_{n}\right\}\right\rangle=\left\{\sum_{i=1}^{n} a_{i} u_{i} \mid a_{i} \in \mathbb{R}\right.$ e $\left.u_{i} \in A, \forall i \in\{1, \ldots, n\}\right\}$.

Seja $V$ um espaço vetorial de dimensão finita munido de $b$ uma forma bilinear nãodegenerada. Dizemos que dois vetores $u, v \in V$ são ortogonais em relação a $b$ desde que, $b(u, v)=0$ e denotamos $u \perp v$. Além disso, dado $A \subset V$ fica definida o conjunto $A^{\perp}=\{v \in V \mid b(v, a)=0 \quad \forall a \in A\}$ dito conjunto ortogonal a $A$. Verifica-se que se $W$ é um subespaço de $V$ então $W^{\perp}$ também é subespaço de $V$.

Dito isto, vemos algumas caracterizações sobre o caráter causal de um subespaço em relação ao seu ortogonal.

Teorema 1.1. Seja $(V, b)$ um espaço métrico tal que a métrica b é não-degenerada e $U \subset V$ é um subespaço vetorial de $V$.

1. $\operatorname{Então}, \operatorname{dim}\left(U^{\perp}\right)=\operatorname{dim}(V)-\operatorname{dim}(U)$;

2. Então, $\left(U^{\perp}\right)^{\perp}=U$;

3. Se U é não-degenerada. Então, $U^{\perp}$ é, também, não-degenerado.

\section{Demonstração:}

1. Seja $m$ a dimensão de $U$ e considere $\left\{\alpha_{1}, \ldots, \alpha_{m}\right\}$ uma base para $U$. Tome uma extensão $\left\{\alpha_{1}, \ldots, \alpha_{m}, \alpha_{m+1}, \ldots, \alpha_{n}\right\}$ como base de $V$. Por linearidade de $b$, temos que um vetor 
$x \in V$ pertence a $U^{\perp}$ se, e somente se, $b\left(e_{i}, x\right)=0$ para cada $i=1, \ldots, m$. Denotando $b_{i j}=b\left(\alpha_{i}, \alpha_{j}\right)$ temos que $x=\sum_{j=1}^{n} x_{j} \alpha_{j} \in V$ pertence a $U^{\perp}$ se, e somente se, é solução do seguinte sistema,

$$
\begin{aligned}
& b_{11} x_{1}+b_{12} x_{2}+\ldots+b_{1 m} x_{m}=0 \\
& b_{21} x_{1}+b_{22} x_{2}+\ldots+b_{2 m} x_{m}=0 \\
& \ldots \\
& b_{n 1} x_{1}+b_{n 2} x_{2}+\ldots+b_{n m} x_{m}=0 .
\end{aligned}
$$

Este é um sistema linear homogêneo de $n$ equações nas $m$ varáveis $x_{j}$. De modo que a dimensão de $U^{\perp}$ coincide com a dimensão do espaço de soluções do sistema acima. Matricialmente este sistema é escrito como

$$
[0]_{n \times 1}=[B]_{n \times m}\left[x_{j}\right]_{m \times 1}
$$

$\operatorname{com} B=\left(b_{i j}\right)$. A dimensão do espaço das soluções do sistema coincide com a dimensão do núcleo da transformação $B: \mathbb{R}^{n} \rightarrow \mathbb{R}^{m}$. Agora, pelo teorema do núcleo e da imagem temos

$$
\operatorname{dim} \mathrm{N}(B)=\operatorname{dim}\left(\mathbb{R}^{n}\right)-\operatorname{dim} \operatorname{Im}(B)=n-m .
$$

Assim, $\operatorname{dim} U^{\perp}=n-m=\operatorname{dim} V-\operatorname{dim} U$. E o resultado está demonstrado.

2. Primeiramente se $x \in U$, então $b(x, v)=0$ para todo $v \in U^{\perp}$, o que ocorre se, e somente se, $x \in\left(U^{\perp}\right)^{\perp}$. Deste modo $U \subset\left(U^{\perp}\right)^{\perp}$. Conforme o item anterior temos

$$
\begin{aligned}
\operatorname{dim}\left(U^{\perp}\right)^{\perp} & =\operatorname{dim} V-\operatorname{dim} U^{\perp} \\
& =\operatorname{dim} V-(\operatorname{dim} V-\operatorname{dim} U) \\
& =\operatorname{dim} U .
\end{aligned}
$$

E assim garantimos que $\left(U^{\perp}\right)^{\perp}=U$.

3. Seja $U$ um subespaço não-degenerado de $V$. Suponhamos por contradição que $U^{\perp}$ é degenerado e tome $\bar{u} \in U^{\perp}$ tal que $\bar{u} \neq 0$ e $b(\bar{u}, v)=0$ para todo $v \in U^{\perp}$, isto é, $\bar{u} \in\left(U^{\perp}\right)^{\perp}=U$ de modo que $U$ é degenerada, um absurdo.

Proposição 1.3. Seja $v \in \mathbb{L}^{3}$. Então, $v$ é tipo-tempo se, e somente se, $\langle\{v\}\rangle^{\perp}$ for tipoespaço. Analogamente $v$ é tipo-espaço se, e somente se, $\langle v\rangle^{\perp}$ for tipo-tempo. Além disso, nos dois casos $\mathbb{L}^{3}=\langle\{v\}\rangle \oplus\langle\{v\}\rangle^{\perp}$. 
Demonstração: Seja $v \in \mathbb{L}^{3}$ um vetor tipo-tempo. Tome $e_{3}=\frac{v}{-\langle v, v\rangle}$ e seja $\left\{e_{1}, e_{2}, e_{3}\right\}$ uma base ortogonal de $\mathbb{L}^{3}$ completada a partir do vetor $e_{3}$, e note que $e_{1}, e_{2}$ deverão ser tipoespaço. Assim, $\langle\{v\}\rangle^{\perp}=\left\langle\left\{e_{1}, e_{2}\right\}\right\rangle$ será tipo-espaço. Reciprocamente seja $\left\{e_{1}, e_{2}\right\}$ vetores ortononais tipo-tempo que geram $\langle\{v\}\rangle^{\perp}$. Estendemos $\left\{e_{1}, e_{2}, e_{3}\right\}$ para uma base ortonormal de $\mathbb{L}^{3}$ de modo que a matriz $g_{i j}$ de $\langle$,$\rangle nesta base terá g_{11}, g_{22}>0$ e $g_{33}<0$ Assim $g(v, v)<0$ donde $v$ é tipo-tempo.

Analogamente verificamos o caso de $u$ tipo-espaço. Além disso, decorre da escolha da base que

$$
\mathbb{L}^{3}=\langle\{v\}\rangle \oplus\langle\{v\}\rangle^{\perp}
$$

Decorre da Proposição 1.3 os dois seguintes corolários;

Corolário 1.1. Seja $U \subset V$. Então, $U$ é tipo-espaço se, e somente se, $U^{\perp}$ é tipo-tempo. Analogamente $U$ é tipo-tempo se, e somente se, $U^{\perp}$ é tipo-espaço.

Corolário 1.2. Seja $U \subset V$. Então, $U$ é tipo-luz se, e somente se, $U^{\perp}$ é tipo-luz.

Proposição 1.4. Sejam $u, v \in \mathcal{L}$ dois vetores tipo-luz. Então, $u$ e $v$ são linearmente dependentes se, e somente se, $\langle u, v\rangle=0$.

Demonstração: Primeiramente se $u=\lambda v$ então, $u \perp v$. Reciprocamente, suponhamos que $u \perp v$. Gostaríamos de mostrar que $u$ é igual a $v$ a menos de um múltiplo escalar.

Considere $\mathbb{L}^{3}=\left\langle\left\{E_{3}\right\}\right\rangle^{\perp} \oplus\left\langle\left\{E_{3}\right\}\right\rangle$ uma decomposição com $E_{3}$ vetor tipo-tempo da base canônica. A menos de um múltiplo escalar podemos escrever $u=x+w$ e $v=y+w$ em que a decomposição $w$ na componente $E_{3}$ é a mesma. Vamos mostrar que $x=y$ e concluir a demonstração. Por um lado como $u$ e $v$ são tipo-luz temos

$$
\begin{aligned}
0 & =\langle u, u\rangle+\langle v, v\rangle \\
& =\langle x, x\rangle+\langle y, y\rangle+2\langle w, w\rangle+2\langle x, w\rangle+2\langle y, w\rangle .
\end{aligned}
$$

Por outro lado, como $0=\langle u, v\rangle$ segue que

$$
\langle w, w\rangle+\langle x, w\rangle+\langle y, w\rangle=-\langle x, y\rangle
$$

Substituindo em 1.4, temos;

$$
0=\langle x, x\rangle+\langle y, y\rangle-2\langle x, y\rangle=\langle x-y, x-y\rangle
$$


Como $x-y \in\left\langle E_{3}\right\rangle^{\perp}$, temos que $x-y$ é tipo-espaço que pela equação devemos ter $x-y=0$. Logo $x=y$ e assim $u$ é igual a $v$ a menos de um múltiplo escalar e o resultado segue.

Proposição 1.5. Seja $U \cap \mathbb{L}^{3}$ um subespaço vetorial de dimensão 2. Então as afirmações abaixo são equivalentes;

1. U contém um vetor tipo-tempo.

2. U é tipo-tempo.

3. U contém dois vetores tipo-luz linearmente independentes.

Demonstração: $(\mathbf{1} \Rightarrow \mathbf{2})$ Seja $u \in U$ um vetor tipo-tempo. Considere $\langle\{u\}\rangle^{\perp}$ que pela Proposição 1.3 é um subespaço tipo-espaço. Como $U^{\perp} \subset\langle\{u\}\rangle^{\perp}$ decorre que $U^{\perp}$ é tipoespaço de modo que o Corolário 1.1 nos garante que $U$ é tipo-tempo.

$(\mathbf{2} \Rightarrow \mathbf{3})$ Suponha que $U$ seja tipo-tempo de onde a métrica $\langle$,$\rangle induzida em U$ é não-degenerada de índice 1. Como $U$ tem dimensão 2 podemos tomar uma base $\left\{e_{1}, e_{2}\right\}$ ortonormal de $U$ tal que $e_{1}$ é tipo-espaço e $e_{2}$ é tipo-tempo. Deste modo os vetores $e_{1}+e_{2}$ e $e_{1}-e_{2}$ serão vetores tipo-luz em $U$, de fato

$$
\left\langle e_{1} \pm e_{2}, e_{1} \pm e_{2}\right\rangle=\left\langle e_{1}, e_{1}\right\rangle \pm 2\left\langle e_{1}, e_{2}\right\rangle+\left\langle e_{2}, e_{2}\right\rangle=1-1=0
$$

Além disso,

$$
\left\{e_{1}+e_{2}, e_{1}-e_{2}\right\}=\left\{e_{1}, e_{1}\right\}-\left\{e_{2}, e_{2}\right\}=1+1=2 \neq 0 .
$$

Logo, decorre da Proposição 1.4 que estes vetores tipo-luz são linearmente independentes.

$(\mathbf{3} \Rightarrow \mathbf{1})$ Sejam $u, v$ dois vetores linearmente independentes tipo-luz em $U$. Nestas condições exatamente um dos vetores $u+v$ ou $u-v$ será um vetor tipo-tempo. De fato,

$$
\langle u \pm v, u \pm v\rangle=\langle u, u\rangle \pm 2\langle u, v\rangle+\langle v, v\rangle= \pm 2\langle u, v\rangle .
$$

Por outro lado, decorre da Proposição 1.4 que $\langle u, v\rangle \neq 0$. Assim, se $\langle u, v\rangle>0$ teremos que $u-v$ é tipo-tempo e se $\langle u, v\rangle<0$, temos que $u+v$ será tipo-tempo.

Proposição 1.6. Seja $U \subset \mathbb{L}^{3}$ um subespaço vetorial. Então, $U$ é tipo-luz se, e somente se, U contém um vetor tipo-luz e não contêm vetores tipo-tempo. 
Demonstração: Seja $U$ um subespaço vetorial de $\mathbb{L}^{3}$. Se $\operatorname{dim} U=3$ então $U=\mathbb{L}^{3}$ e consequentemente não pode ser tipo-luz. Se $\operatorname{dim} U=1$ então a afirmação vale notando que $U$ será gerado por um vetor tipo-luz. Por último suponhamos que $\operatorname{dim} U=2$. Assim, se $U$ é tipo-luz, temos que a métrica $\langle$,$\rangle restrita a U$ é degenerada e existe um vetor $v \neq 0$ tal que $\langle v, u\rangle=0$ para todo $u \in U$. Em particular $\langle v, v\rangle=0$ e segue que $v \in U$ é tipo-luz. Além disso decorre da Proposição 1.5 que $U$ não contêm vetores tipo-tempo. Reciprocamente, se $U$ não contêm vetores tipo-tempo a Proposição 1.5 nos garante que $U$ não é tipo-tempo. Supondo que $U$ contém um vetor tipo-luz temos que $U$ não pode ser tipo-espaço. Assim temos que $U$ deve ser tipo-luz e o resultado segue.

\subsubsection{Propriedades geométricas dos vetores em $\mathbb{L}^{3}$}

Nesta seção trataremos da "Geometria Analítica" no espaço de Minkowski, isto é, traremos as definições e propriedades básicas dos vetores neste espaço, como por exemplo os conceitos de norma de vetores, ângulo entre vetores e produto vetorial.

Na subsção anterior fornecemos caracterizações de subespaços vetoriais em $\mathbb{L}^{3}$ quanto ao seus caracteres causais. Com isto fica definido o caráter causal de Retas $(R)$ e de planos $(P)$ no espaço de Minkowski, conforme segue.

Definição 1.10. Sejam $R \subset \mathbb{R}^{3}$ uma reta e $P \subset \mathbb{R}^{3}$ um plano no espaço de Minkowski. Então dizemos que;

1. $R \subset \mathbb{R}^{3}$ é uma reta tipo-espaço, tipo-tempo ou tipo-luz desde que, o subespaço vetorial unidimensional, é respectivamente tipo-espaço, tipo-tempo ou tipo-luz.

2. $P \subset \mathbb{R}^{3}$ é um plano tipo-espaço (ou Riemanniano), tipo-tempo (ou Lorentziano) ou tipo-luz (ou Degenerado) desde que, o subespaço vetorial bidimensional, é respectivamente tipo-espaço, tipo-tempo ou tipo-luz.

Com esta definição, podemos usar as caracterizações de subespaços vetoriais uni-dimensionais e bi-dimensionais para caracterizar retas e planos.

Proposição 1.7. Sejam $P \subset \mathbb{R}^{3}$ um plano e $\vec{n}_{\mathbb{E}^{3}} \in \mathbb{R}^{3}$ um vetor normal, em relação a métrica euclidiana, ao plano P. Então, P é tipo-espaço, tipo-tempo ou tipo-luz se, e somente se, $\vec{n}_{\mathbb{E}^{3}}$ é tipo-espaço, tipo-tempo ou tipo-luz respectivamente. 
Demonstração: De fato, se $P \subset \mathbb{R}^{3}$ é um plano com $\vec{n}_{\mathbb{E}^{3}}=(a, b, c)$ um vetor normal a este plano em relação a métrica euclidiana, podemos escrever $\bar{P}=\left\{(x, y, z) \in \mathbb{R}^{3} \mid a x+b y+c z=0\right\}$ em que $\bar{P}$ é a translação de $P$ a origem de $\mathbb{R}^{3}$. Como

$$
\bar{P}=\left\{(x, y, z) \in \mathbb{R}^{3} \mid a x+b y-(-c) z=0\right\}=\langle\{(a, b,-c)\}\rangle^{\perp},
$$

segue da Proposição (1.3) que o caráter causal de $P$ coincide com o caráter causal de $(a, b,-c)$ que por sua vez é possui o mesmo caráter de $\vec{n}_{\mathbb{E}^{3}}$, de fato note que

$$
\langle(a, b,-c),(a, b,-c)\rangle=a^{2}+b^{2}-(-c)^{2}=a^{2}+b^{2}-c^{2}=\left\langle\vec{n}_{\mathbb{E}^{3}}, \vec{n}_{\mathbb{E}^{3}}\right\rangle .
$$

E assim a Proposição está demonstrada.

Convém darmos a seguinte definição.

Definição 1.11. Seja $u \in \mathbb{L}^{3}$ um vetor tipo-tempo no espaço de Minkowski. Definimos o cone tipo-tempo do vetor $u$, pelo conjunto $\mathcal{C}(u)=\left\{v \in \mathbb{L}^{3} \mid v \in \mathcal{T},\langle u, v\rangle \leqslant 0\right\}$.

Assim, note que dado um vetor $u \in \mathcal{T}$ tipo-tempo em $\mathbb{L}^{3}$, todos os vetores tipo-tempo ficam distribuídos em duas classes (de equivalência) $\mathcal{C}(u)$ e $\mathcal{C}(-u)$. Ainda costuma-se dizer que os vetores tipo-tempo que pertencem ao cone tipo-tempo do vetor $(0,0,1)$ apontam para o futuro enquanto os vetores tipo tempo pertencentes a $\mathcal{C}(-(0,0,1))$ apontam para o passado.

Definimos também o módulo de um vetor de $\mathbb{L}^{3}$.

Definição 1.12. Seja $u \in \mathbb{L}^{3}$ um vetor no espaço de Minkowski, definimos a norma de $u$ pelo número real, não negativo, dado por; $|u|=\sqrt{|\langle u, u\rangle|}$. Além disso dizemos que $u \in \mathbb{L}^{3}$ é um vetor unitário, desde que sua norma seja igual a 1.

Notação 1.1. Seja $u \in \mathbb{R}^{3}$ um vetor, denotaremos $|u|_{\mathbb{E}^{3}}=\sqrt{\langle u, u\rangle_{\mathbb{E}^{3}}}$ pela norma de $u$ em relação a métrica euclidiana.

Exemplo 1.1. Se $u=(x, y, z) \in \mathbb{L}^{3}$ é um vetor tipo-espaço, então $x^{2}+y^{2}-z^{2} \geq 0$, de modo que $|u|=\sqrt{\left|x^{2}+y^{2}-z^{2}\right|}=\sqrt{x^{2}+y^{2}-z^{2}}$. Assim $u$ é um vetor tipo-espaço unitário desde que $1=x^{2}+y^{2}-z^{2}$.

Denotaremos por o conjunto de todos os vetores unitários tipo-espaço por $\mathbf{S}^{2}$;

$$
\mathbf{S}^{2}=\left\{(x, y, z) \in \mathbb{L}^{3} \quad \mid \quad x^{2}+y^{2}-z^{2}=1\right\}
$$


Geometricamente este conjunto de pontos forma uma superfície chamada espaço de De Sitter.

Exemplo 1.2. Se $u=(x, y, z) \in \mathbb{L}^{3}$ é um vetor tipo-tempo, então $x^{2}+y^{2}-z^{2}<0$, de modo que $|u|=\sqrt{\left|x^{2}+y^{2}-z^{2}\right|}=\sqrt{-x^{2}-y^{2}+z^{2}}$. Assim $u$ é um vetor tipo-tempo unitário desde que $x^{2}+y^{2}-z^{2}=-1$.

Denotaremos por o conjunto de todos os vetores unitários tipo-tempo por $\mathbf{H}^{2}$;

$$
\mathbf{H}^{2}=\left\{(x, y, z) \in \mathbb{L}^{3} \quad \mid \quad x^{2}+y^{2}-z^{2}=-1\right\}
$$

Geometricamente este conjunto de pontos formam duas superfícies chamadas de plano hiperbólico.

Lembremos que para dois vetores $u, v \in \mathbb{E}^{3}$, no espaço Euclidiano, vale a desigualdade de Cauchy-Schwarz, que nos assegura que;

$$
\left|\langle u, v\rangle_{\mathbb{E}^{3}}\right| \leqslant|u|_{\mathbb{E}^{3}}|v|_{\mathbb{E}^{3}}
$$

Em que a igualdade ocorre se, e somente se, os vetores $u$ e $v$ são proporcionais. Acontece que para norma no espaço de Minkowski esta desigualdade não se verifica. Na verdade temos uma desigualdade semelhante para vetores do tipo-tempo, conforme o Teorema a seguir.

Teorema 1.2. Sejam $u, v \in \mathbb{L}^{3}$ dois vetores tipo-tempo no espaço de Minkowski, então;

$$
|\langle u, v\rangle| \geqslant|u||v|,
$$

e a igualdade se verifica se, e somente se, u e v são proporcionais. Chamamos esta relação de desigualdade reversa de Cauchy-Schwarz.

Demonstração: De fato, sejam $u, v$ vetores em $\mathbb{L}^{3}$. Suponhamos primeiramente que estes vetores sejam linearmente independentes. Consideremos então $P=\langle\{u, v\}\rangle$ o plano tipotempo gerado por $u$ e $v$. Assim a Proposição 1.5 nos assegura a existência de pelo menos dois vetores tipo-luz no plano $P$ de modo que para pelo menos um par de coeficientes, digamos $a$ e $b$, deve-se verificar que

$$
\langle a u+b v, a u+b v\rangle=a^{2}\langle u, u\rangle+2 a b\langle u, v\rangle+b^{2}\langle v, v\rangle=0 .
$$

Como $a \neq 0$ (caso contrário isto implicaria que $v$ é tipo-luz) esta última equação é equivalente a

$$
\langle u, u\rangle+2 \lambda\langle u, v\rangle+\lambda^{2}\langle v, v\rangle=0
$$


com $\lambda=b / a$. Como esta equação, na variável $\lambda$, admite pelo menos duas raízes reais e ao mesmo tempo é um polinômio de segundo grau na variável $\lambda$, devemos ter que seu discriminante é estritamente positivo, isto é,

$$
\triangle=(2\langle u, v\rangle)^{2}-4\langle v, v\rangle\langle u, u\rangle>0
$$

Assim,

$$
|\langle u, v\rangle|>\langle u, u\rangle\langle v, v\rangle
$$

e verificamos a desigualdade.

Por último suponhamos que os vetores $u$ e $v$ são linearmente dependentes, assim existe um número real $\xi \in \mathbb{R}$ tal que $v=\xi u$ e então

$$
|\langle u, v\rangle|=|\xi||\langle u, u\rangle|=|\xi||u|^{2}=|u||v|
$$

e assim verificamos a igualdade. Reciprocamente se a igualdade se verifica temos pelo caso anterior que $u$ e $v$ não podem ser linearmente independentes. E assim o teorema está demonstrado.

Definimos também o produto vetorial em $\mathbb{L}^{3}$ da mesma maneira que é definido no espaço Euclidiano $\mathbb{E}^{3}$.

Definição 1.13. Dados dois vetores $u, v \in \mathbb{L}^{3}$ dizemos que o produto vetorial (Lorentiziano) entre $u$ e $v$, denotado por $u \times v$, é o único vetor em $\mathbb{L}^{3}$ satisfazendo

$$
\langle u \times v, w\rangle=\operatorname{det}(u, v, w), \quad \forall w \in \mathbb{L}^{3} .
$$

Em que,

$$
\operatorname{det}(u, v, w)=\left|\begin{array}{ccc}
u_{1} & u_{2} & u_{3} \\
v_{1} & v_{2} & v_{3} \\
w_{1} & w_{2} & w_{3}
\end{array}\right|,
$$

e $u_{i}, v_{i}$ e $w_{i}$ são as coordenadas dos vetores $u, v$ e $w$ em relação a base canônica.

Observação 1.1. Como definido o produto, $u \times v$, entre dois vetores $u$ e $v$ é único. De fato, suponha que exista $\bar{u}$ tal que $\langle\bar{u}, w\rangle=\operatorname{det}(u, v, w)$ para todo $w$. Isto significa que para cada vetor $w$ deverá se verificar que $\langle\bar{u}, w\rangle-\langle u \times v, w\rangle=0$, de modo que $\langle\bar{u}-u \times v, w\rangle=0$, o que implica em $\bar{u}=u \times v$. 
Tomando $w$ na definição acima como elementos da base canônica $\left\{e_{1}, e_{2}, e_{3}\right\}$ o produto lorentziano assume a seguinte forma:

$$
u \times v=\left|\begin{array}{rrr}
e_{1} & e_{2} & -e_{3} \\
u_{1} & u_{2} & u_{3} \\
v_{1} & v_{2} & v_{3}
\end{array}\right|=\left|\begin{array}{cc}
u_{2} & u_{3} \\
v_{2} & v_{3}
\end{array}\right| e_{1}-\left|\begin{array}{cc}
u_{1} & u_{3} \\
v_{1} & v_{3}
\end{array}\right| e_{2}-\left|\begin{array}{cc}
u_{1} & u_{2} \\
v_{1} & v_{2}
\end{array}\right| e_{3} .
$$

E segue da definição e das propriedades do determinante as seguintes propriedades para o produto vetorial.

Propriedades 1.1. Sejam $u, v, w \in \mathbb{L}^{3}$ e $\lambda, \mu \in \mathbb{R}$, o produto vetorial satisfaz:

1. Bilinearidade: $u \times(\lambda v+\mu w)=\lambda(u \times v)+\mu(u \times w) e$ $(\lambda u+\mu v) \times w=\lambda(u \times w)+\mu(v \times w)$.

2. Anti-comutatividade; $u \times v=-v \times u$.

3. Produto misto: $\langle u \times v, w\rangle=\langle u, v \times w\rangle$.

4. Ortogonalidade: $\langle u \times v, u\rangle=\langle u \times v, v\rangle=0$.

5. $u \times v=0$ se, e somente se, $u$ e $v$ são proporcionais.

6. se $u, v, x, y \in \mathbb{L}^{3}$ então,

$$
\langle u \times v, x \times y\rangle=\operatorname{det}\left(\begin{array}{cc}
\langle u, x\rangle & \langle v, x\rangle \\
\langle u, y\rangle & \langle v, y\rangle
\end{array}\right)
$$

7. Regra de Leibniz: se u e $v$ são funções vetoriais de uma variável real, então, $(u \times v)^{\prime}=$ $u^{\prime} \times v+u \times v^{\prime}$.

Por último enceraremos esta seção tratando de ângulos entre vetores no espaço de Minkowski. É um fato notável, que ao contrário do espaço Euclidiano, nem sempre é possível definir um ângulo entre vetores que mantenha propriedades geométricas de interesse.

Por exemplo se $u$ e $v$ dois vetores em $\mathbb{E}^{3}$ sabemos que o ângulo, digamos $\phi_{\mathbb{E}^{3}}$ é caracterizado pela relação

$$
\cos \left(\phi_{\mathbb{E}^{3}}\right)=\frac{\langle u, v\rangle_{\mathbb{E}^{3}}}{|u|_{\mathbb{E}^{3}|v|_{\mathbb{E}^{3}}}}
$$

Tal relação implica propriedades entres vetores, como por exemplo o sinal do produto $\langle u, v\rangle_{\mathbb{E}^{3}}$ está relacionado como ângulo $\phi_{\mathbb{E}^{3}}$ ser agudo ou obtuso.

Em alguns casos fica bem definido um ângulo entre vetores que preserva grande parte das propriedades geométricas de interesse. Como por exemplo, se dois vetores tipo-tempo estão 
no mesmo cone tipo-tempo, a desigualdade reversa de Cauchy-Schwarz nos permite definir um tipo de ângulo;

Proposição 1.8. Sejam $u, v \in \mathbb{L}^{3}$ dois vetores tipo-tempo no espaço de Minkowki tais que $v \in \mathcal{C}(u)$. Então existe um único número real não negativo $\phi$, tal que

$$
\langle u, v\rangle=-|u||v| \cosh \phi
$$

Demonstração: De fato, pela desigualdade reversa de Cauchy-Schwarz, temos que $|\langle u, v\rangle| \geqslant$ $|u||v|$. Supondo que $u$ e $v$ estão na mesmo cone tipo-tempo temos que $\langle u, v\rangle<0$ e assim temos a seguinte expressão;

$$
\frac{-\langle u, v\rangle}{|u||v|} \geqslant 1
$$

de modo que, sendo a função co-seno hiperbólico biunívoca, existe um único número real $\phi \in[0+\infty)$ tal que, $\langle u, v\rangle=-|u||v| \cosh \phi$.

Definição 1.14. Sejam dois vetores $u, v \in \mathbb{L}^{3}$ tipo-tempo tais que $v \in \mathcal{C}(u)$. Então, o número $\phi \in[0+\infty)$ tal que satisfaz a Equação (1.7) é dito Ângulo hiperbólico entre u e $v$.

Podemos, ainda, definir uma aplicação ângulo para outros casos da seguinte maneira, dados dois vetores $u$ e $v$ linearmente independentes no espaço de Minkowski, então fica definido um plano $P=\langle\{u, v\}\rangle$ em $\mathbb{L}^{3}$ que pode ser tipo-espaço, tipo-tempo ou tipo-luz.

\section{Caso 1: $P$ é Rimemmaniano.}

Isto é, $P$ é um plano tipo-espaço. Deste modo garantimos que a métrica em $P$ é positivadefinida de modo que o plano $P$ com a métrica induzida é uma "cópia" de $\mathbb{R}^{2}$ munido do produto interno Euclidiano $\mathbb{E}^{2}=\left(\mathbb{R}^{2},\langle,\rangle_{\mathbb{R}^{2}}\right)$. Assim definimos o ângulo entre $u$ e $v$ pelo ângulo Euclidiano entre $u$ e $v$, isto é $\varangle(u, v)$ é o único número $0 \leq \theta \leq \pi$ tal que;

$$
\cos \theta=\frac{\langle u, v\rangle}{|u||v|}
$$

Observação 1.2. O leitor já familiarizado com o conceito de Superfícies Abstratas notará que esta "cópia" é na verdade uma aplicação isométrica entre $\left(P,\left.\langle\rangle\right|_{P},\right)$ e $\mathbb{E}^{2}$. Além disso irá notar que, como uma isometria preserva propriedades geométricas, este ângulo estará bem definido. 


\section{Caso 2: $P$ é Lorentziano.}

Isto é, $P$ é um plano tipo-tempo. Deste modo métrica induzida em $P$ é não-degenerada de índice 1, e assim $P$ será uma " cópia" do espaço bidimensional de Minkowski $\mathbb{L}^{2}=\left(\mathbb{R}^{2},\langle,\rangle_{\mathbb{L}^{2}}\right)$, em que a métrica é;

$$
\left(x_{1}, x_{2}\right),\left(y_{1}, y_{2}\right) \in \mathbb{R}^{2} \mapsto\left\langle\left(x_{1}, x_{2}\right),\left(y_{1}, y_{2}\right)\right\rangle_{\mathbb{L}^{2}}=x_{1} y_{1}-x_{2} y_{2} .
$$

Denote por $\mathbb{U}^{2}=\left\{\left.v \in \mathbb{L}^{2}|| v\right|_{\mathbb{L}^{2}}=1\right\}$ o conjunto dos vetores unitários de $\mathbb{L}^{2}$. É possível verificar que este conjunto se decompõe em quatro componentes conexas:

$$
\begin{gathered}
\mathbb{H}_{+}^{1}=\left\{(x, y) \in \mathbb{L}^{2} \mid x^{2}-y^{2}=-1, y>0\right\}, \quad \mathbb{H}_{-}^{1}=\left\{(x, y) \in \mathbb{L}^{2} \mid x^{2}-y^{2}=-1, y<0\right\}, \\
\mathbb{S}_{+}^{1}=\left\{(x, y) \in \mathbb{L}^{2} \mid x^{2}-y^{2}=+1, y>0\right\} \quad \text { e } \quad \mathbb{S}_{-}^{1}=\left\{(x, y) \in \mathbb{L}^{2} \mid x^{2}-y^{2}=+1, y<0\right\} .
\end{gathered}
$$

Deste modo se $u$ e $v$ por esta estiverem na mesma componente conexa fica definindo um ângulo hiperbólico entre eles de modo análogo ao que definimos na Definição (1.14).

Observação 1.3. O sentido de "cópia" aqui é o mesmo que na Observação 1.2 . Sendo que neste caso a aplicação de isometria será entre $\left(P,\left.\langle\rangle\right|_{P},\right)$ e $\mathbb{L}^{2}$. Além disso o leitor poderá verificar que a isometria $(x, y) \mapsto(y, x)$ em $\mathbb{L}^{2}$ mapeia $\mathbb{H}^{1}$ em $\mathbb{S}^{1}$, isto é transforma vetores tipo-espaço (respectivamente tipo-tempo) em vetores tipo-tempo (respectivamente tipo-espaço) de modo que fica bem definido o ângulo hiperbólico entre vetores tipo-espaço em $\mathbb{S}^{1}$.

\section{Caso 3: $P$ é Degenerado.}

Isto é, $P$ é um plano tipo-luz. Neste caso não definimos nenhuma aplicação tipo ângulo entre os vetores $u$ e $v$.

\subsubsection{Teoria local das superfícies no Espaço de Minkowski}

Nesta seção trataremos das superfícies parametrizadas no espaço de Minkowski. Escolhemos fazer uma abordagem local via o conceito de Superfícies Parametrizadas, de modo que estaremos interessados em definir tais objetos e estabelecer algumas de suas propriedades geométricas como a curvatura Gaussiana e curvatura média. 
Definição 1.15. Seja $\mathbb{X}: U \subset \mathbb{R}^{2} \rightarrow \mathbb{L}^{3}$ uma aplicação definida em um subconjunto $U$ aberto e conexo de $\mathbb{R}^{2}$. Dizemos que $\mathbb{X}$ é uma Superfície parametrizada em $\mathbb{L}^{3}$ desde que, $\mathbb{X}$ seja diferenciável em $U$.

Definição 1.16. Seja $\mathbb{X}: U \subset \mathbb{R}^{2} \rightarrow \mathbb{R}^{3}$ é uma superfície parametrizada. Dizemos que $\mathbb{X} e ́$

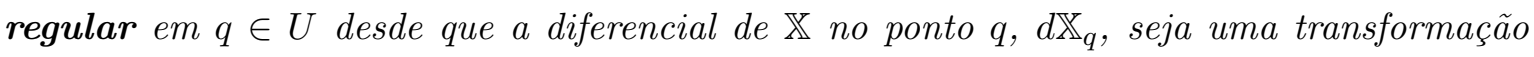
linear injetiva, e dizemos que $\mathbb{X}$ é regular desde que, seja regular em cada ponto de $U$. Dizemos que $\mathbb{X}$ é simples desde que, o traço da aplicação $\mathbb{X}(U) \subset \mathbb{R}^{3}$ não admita autointerseção.

Dada uma superfície parametrizada regular $\mathbb{X}: U \subset \mathbb{R}^{2} \rightarrow \mathbb{L}^{3}$ no espaço de Minkowski a condição de regularidade nos garante que em cada ponto $q_{0} \in U$ é possível associar um espaço vetorial de dimensão 2, chamado plano tangent£ $\rrbracket^{1}$.

Definição 1.17. Seja $\mathbb{X}: U \subset \mathbb{R}^{2} \rightarrow \mathbb{L}^{3}$ uma superfície parametrizada regular. Dado $q_{0}=\left(u_{0}, v_{0}\right) \in U$ dizemos que $w \in \mathbb{L}^{3}$ é um vetor tangente a superfície $\mathbb{X}$ em $q_{0}$, desde que $w$ seja dado por $w=\alpha^{\prime}\left(t_{0}\right)$, em que $\alpha(t)=\mathbb{X}(u(t), v(t))$ é a imagem por $\mathbb{X}$ de alguma curva $(u(t), v(t))$ em $U$ com $\left(u\left(t_{0}\right), v\left(t_{0}\right)\right)=q_{0}$. Denotaremos por $T_{q} \mathbb{X}$ o conjunto de todos vetores tangentes a superfície $\mathbb{X}$ em $q$ e diremos que $T_{q} \mathbb{X}$ é o plano tangente a $\mathbb{X}$ em $q \in U$.

Deste modo temos que $\mathbb{X}_{u}\left(q_{0}\right)$ e $\mathbb{X}_{v}\left(q_{0}\right)$ serão vetores tangentes as curvas coordenadas $\mathbb{X}\left(u, v_{0}\right)$ e $\mathbb{X}\left(u, v_{0}\right)$ respectivamente. Analogamente as superfícies no espaço Euclidiano, por injetividade da diferencial $d \mathbb{X}_{q}$ os vetores $\mathbb{X}_{u}\left(q_{0}\right)$ e $\mathbb{X}_{v}\left(q_{0}\right)$, ditos vetores tangentes coordenados, formam uma base para o plano tangente a superfície em $q_{0}$. Diremos que $\left\{\mathbb{X}_{u}(q), \mathbb{X}_{v}(q)\right\}$ é a base coordenada do plano tangente a superfície em $q \in U$.

Como o plano tangente é um subespaço vetorial de $\mathbb{L}^{3}$ podemos introduzir um produto interno neste espaço, e isto de certa forma permitirá introduzir conceitos métricos na superfície. Dada uma superfície parametrizada regular no espaço de Minkowski, muniremos cada plano tangente com a métrica de Lorentz $g$ induzida de $\mathbb{L}^{3}$ da seguinte forma;

$$
g: T_{q} \mathbb{X} \times T_{q} \mathbb{X} \rightarrow \mathbb{R} ; \quad(v, w) \mapsto g(v, w)=\langle v, w\rangle
$$

De modo que cada plano tangente será também um espaço vetorial de dimensão dois munido da métrica $g$. Esta métrica, como induzida $\langle$,$\rangle , é uma forma bilinear simétrica, e a$ ela podemos corresponder a um forma quadrática conforme a

\footnotetext{
${ }^{1}$ Observemos que tais noções de superfícies, vetores tangentes e planos tangentes não são conceitos métricos, de modo que repetiremos as mesmas definições dada nos livros de Geometria Diferencial no espaço Euclidiano, como por exemplo, na seção 3 do capítulo 3 de 20 .
} 
Definição 1.18. Seja $\mathbb{X}: U \subset \mathbb{R}^{2} \rightarrow \mathbb{L}^{3}$ uma superfície parametrizada regular e $T_{q} \mathbb{X}$ o plano tangente a $\mathbb{X}$ em $q$. A forma quadrática

$$
I_{q}: T_{q} \mathbb{X} \rightarrow \mathbb{R} ; \quad w \in T_{q} \mathbb{X} \mapsto I_{q}(w)=g(w, w)=\langle w, w\rangle \in \mathbb{R}
$$

é dita primeira forma fundamental de $\mathbb{X}$ em $q \in U$.

E assim poderemos definir caráter causal para uma superfície parametrizada regular de acordo com o caráter causal do plano tangente.

Definição 1.19. Seja $\mathbb{X}: U \in \mathbb{R}^{e} \rightarrow \mathbb{L}^{3}$ uma superfície parametrizada regular no espaço de Minkowski, e $q \in U$. Dizemos que:

1. A superfície $\mathbb{X}$ é tipo-espaço (respectivamente tipo-tempo) em q desde que, o plano tangente $T_{q} \mathbb{X}$ a $\mathbb{X}$ em q seja um plano tipo-espaço (respectivamente tipo-tempo).

2. A superfície $\mathbb{X}$ é tipo-luz ou degenerada em q desde que, o plano tangente $T_{q} \mathbb{X}$ a $\mathbb{X}$ em q seja um plano tipo-luz.

Dizemos também que $\mathbb{X}$ é superfície tipo-espaço (respectivamente tipo-tempo, tipo-luz ou degenerada) (resp. tipo-tempo, tipo-luz) desde que seja tipo-espaço (respec. tipo-tempo, tipo-luz) em cada $q \in U$.

Dada $\mathbb{X}$ uma superfície parametrizada regular no espaço de Minkowski, podemos expressar a primeira forma quadrática em termos da base coordenada $\left\{\mathbb{X}_{u}, \mathbb{X}_{v}\right\}$ da seguinte forma: se $w \in T_{q} \mathbb{X}$ então $w=a \mathbb{X}_{u}+b \mathbb{X}_{v}$ para algum par $a$ e $b$ de números reais, de modo que

$$
\begin{aligned}
I_{q}(w) & =\left\langle a \mathbb{X}_{u}+b \mathbb{X}_{v}, a \mathbb{X}_{u}+b \mathbb{X}_{v}\right\rangle=a^{2}\left\langle\mathbb{X}_{u}, \mathbb{X}_{u}\right\rangle+2 a b\left\langle\mathbb{X}_{u}, \mathbb{X}_{v}\right\rangle+b^{2}\left\langle\mathbb{X}_{v}, \mathbb{X}_{v}\right\rangle \\
& :=a^{2} E+2 a b F^{2}+b^{2} G,
\end{aligned}
$$

em que, as funções $E, F, G: U \subset \mathbb{R}^{2} \rightarrow \mathbb{L}^{3}$ são dadas respectivamente por:

$$
E(q)=\left\langle\mathbb{X}_{u}(q), \mathbb{X}_{u}(q)\right\rangle, F(q)=\left\langle\mathbb{X}_{u}(q), \mathbb{X}_{v}(q)\right\rangle, G(q)=\left\langle\mathbb{X}_{v}(q), \mathbb{X}_{v}(q)\right\rangle
$$

E são ditas coeficientes da primeira forma fundamental.

A matriz de $I$ expressa na base coordenada é dada por:

$$
I_{q} \doteq\left(\begin{array}{cc}
E(q) & F(q) \\
F(q) & G(q)
\end{array}\right) .
$$

Assim, temos uma caracterização do caráter causal de uma superfície não-degenerada em termos dos coeficientes da primeira forma fundamental. 
Proposição 1.9. Seja $\mathbb{X}: U \subset \mathbb{R}^{2} \rightarrow \mathbb{L}^{3}$ uma superfície parametrizada regular não-degenerada no espaço de Minkowski com E,F e G coeficientes da primeira forma fundamental. Então $\mathbb{X}$ é tipo-espaço (respectivamente tipo-tempo) se, e somente se, $E G-F^{2}>0$ (respectivamente $<0)$.

Demonstração: Suponhamos que $\mathbb{X}$ seja não-degenerada. Tome uma base $\left\{\alpha_{1}, \alpha_{2}\right\}$ ortonormal de $T_{q} \mathbb{X}$ e considere $\left(\alpha_{i j}\right)$ a matriz representativa da forma $\left.g\right|_{T_{q} \mathbb{X}}$. Podemos supor que

$$
\begin{aligned}
& \left(g_{i j}\right)=\left(\begin{array}{cc}
1 & 0 \\
0 & 1
\end{array}\right), \quad \text { se a superfície é tipo-espaço; } \\
& \left(g_{i j}\right)=\left(\begin{array}{cc}
1 & 0 \\
0 & -1
\end{array}\right), \quad \text { se a superfície é tipo-tempo. }
\end{aligned}
$$

Pela Proposição 1.2 garantimos que a matriz representativa de $\left.g\right|_{T_{q} \mathbb{X}}$, na base coordenada, dada pela Equação (1.9), também é inversível, donde o determinante desta matriz é $\operatorname{det}\left(g_{i j}\right)=$ $E G-F^{2}$ é diferente de zero. Seja $M$ a matriz de passagem das base coordenada para a base $\left\{\alpha_{1}, \alpha_{2}\right\}$, e segue da Equação 1.2 que

$$
\left(g_{i j}\right)=M^{t}\left(\alpha_{i j}\right) M
$$

Tomando o determinante desta expressão, temos $\operatorname{det}\left(g_{i j}\right)=(\operatorname{det} M)^{2} \operatorname{det}\left(\alpha_{i j}\right)$ e consequentemente;

$$
E G-F^{2}=(\operatorname{det} M)^{2} \operatorname{det}\left(\alpha_{i j}\right),
$$

como $(\operatorname{det} M)^{2}>0$ e também $\operatorname{det}\left(\alpha_{i j}\right)=1>0$ se $\mathbb{X}$ é tipo-espaço ou $\operatorname{det}\left(\alpha_{i j}\right)=-1<0$ se $\mathbb{X}$ é tipo-tempo, temos que o resultado segue.

Lembramos que grande parte dos aspectos geométricos das superfícies no espaço Euclidiano, provém da aplicação normal de gauss, que mapeia cada cada ponto da superfície na esfera euclidiana de raio unitário dada pelo vetor normal àquele ponto. Adaptando-se o conceito de vetor unitário para o espaço de Minkowski, podemos também, definir o conceito de aplicação normal de gauss em $\mathbb{L}^{3}$.

Podemos sempre definir localmente a aplicação normal de gauss $N$ para superfícies nãodegeneradas no espaço de Minkowski, por:

$$
N: U \subset \mathbb{R}^{2} \rightarrow \mathbb{L}^{3} ; \quad N(u, v)=\frac{\mathbb{X}_{u} \times \mathbb{X}_{v}}{\left|\mathbb{X}_{u} \times \mathbb{X}_{v}\right|}(u, v)
$$


Se $\mathbb{X}$ é uma superfície não-degenerada o vetor normal, $N(q)$ à superfície em $q \in U$, será tipo-espaço ou tipo-tempo dependendo se a superfície é respectivamente tipo-tempo ou tipoespaço em $q \in U$. Como o caráter causal de uma superfície é uma propriedade local, isto é, dado $q \in U$ tipo-espaço (ou tipo-tempo) existirá uma vizinhança de $q$ tal que todos os pontos desta vizinhança será tipo-espaço (ou tipo-tempo), podemos definir, pelo menos localmente, a aplicação normal de gauss, notando que o Plano Hiperbólico $\mathbb{H}^{2}(1.6)$ e Espaço de De Sitter $\mathbb{S}^{2}$ (1.5), formam o análogo, no espaço de Minkowski, à esfera unitária no espaço Euclidiano;

Definição 1.20. Seja $\mathbb{X}: U \in \mathbb{R}^{2} \rightarrow \mathbb{L}^{3}$ uma superfície não-degenerada. Definimos localmente a Aplicação Normal de Gauss por:

1. $N: U \subset \mathbb{R}^{2} \rightarrow \mathbb{H}^{2} ; q \mapsto N(q) \in \mathbb{H}^{2}$ na vizinhança de pontos em que a superfície é tipo-espaço.

2. $N: U \subset \mathbb{R}^{2} \rightarrow \mathbb{S}^{2} ; q \mapsto N(q) \in \mathbb{S}^{2}$ na vizinhança de pontos em que a superfície é tipo-tempo.

Observação 1.4. Notamos que se $\mathbb{X}$ é uma superfície não-degenerada podemos reduzir, se necessário, o aberto $U$ de modo a supor que $\mathbb{X}$ é tipo-espaço ou tipo-tempo, e deste modo definir globalmente a aplicação normal de gauss.

Conforme a Propriedade 6 de (1.1) do produto vetorial, podemos mostrar que

$$
\left\langle\mathbb{X}_{u} \times \mathbb{X}_{v}, \mathbb{X}_{u} \times \mathbb{X}_{v}\right\rangle=\left\langle\mathbb{X}_{u} \times \mathbb{X}_{u}\right\rangle\left\langle\mathbb{X}_{v}, \mathbb{X}_{v}\right\rangle-\left\langle\mathbb{X}_{u}, \mathbb{X}_{v}\right\rangle^{2}
$$

Logo, $\left|\mathbb{X}_{u} \times \mathbb{X}_{v}\right|=\sqrt{-\epsilon\left(E G-F^{2}\right)}$ e assim podemos expressar

$$
N=\frac{\mathbb{X}_{u} \times \mathbb{X}_{v}}{\sqrt{-\epsilon\left(E G-F^{2}\right)}}
$$

A aplicação normal de gauss é diferenciável e sua diferencial em um ponto $q \in U$ é uma aplicação linear que corresponde a cada vetor $V \in \mathbb{R}^{2}$ a derivada direcional na direção de $V$, isto é,

$$
d N_{q}(V)=\lim _{t \rightarrow 0} \frac{N(q+t V)-N(q)}{t}=(N \circ \alpha)^{\prime}(0),
$$

com $\alpha(t)=q+t V \in U$. E sendo $N \circ \alpha(t)$ um vetor normal em $\mathbb{L}^{3}$ perpendicular ao plano tangente, garantimos que $(N \circ \alpha)^{\prime}$ pertence ao plano tangente, isto é, $d N_{q}(V) \in T_{q} \mathbb{X}$ para todo $V \in \mathbb{R}^{2}$. 
Definição 1.21. Seja $\mathbb{X}: U \subset \mathbb{R}^{2} \rightarrow \mathbb{L}^{3}$ uma superfície não-degenerada no espaço de Minkowski. Dado $q \in U$ definimos o operador;

$$
S_{q}: T_{q} \mathbb{X} \rightarrow T_{q} \mathbb{X} ; \quad w \in T_{q} \mathbb{X} \mapsto S_{q}(w)=-d N_{q}\left(d \mathbb{X}_{q}^{-1} w\right) \in T_{q} \mathbb{X}
$$

como sendo o Operador de Weingarten ou Operador Forma da superfície $\mathbb{X}$ em $q$.

Proposição 1.10. Seja $\mathbb{X}: U \subset \mathbb{R}^{2} \rightarrow \mathbb{L}^{3}$ uma superfície paramentrizada regular no espaço de Minkowski. Então em cada $q \in U$ o operador de Weingarten $S_{q}$ de $\mathbb{X}$ é um operador auto-adjunto em relação ao produto interno de Lorentz.

Demonstração: Recordamos que $S_{q}$ será um operador auto-adjunto em $T_{q} \mathbb{X}$ desde que, seja uma aplicação linear neste espaço satisfazendo

$$
\left\langle S_{q}(v), w\right\rangle=\left\langle v, S_{q}(w)\right\rangle, \quad \forall v, w \in T_{q} \mathbb{X}
$$

Como $d N_{q}$ é uma aplicação linear, o operador de Weingarten também o é. Assim nos resta mostrar que $s_{q}$ satisfaz a propriedade acima.

Expressamos, na base coordenada, os vetores tangentes $x=x_{1} \mathbb{X}_{u}+x_{2} \mathbb{X}_{v}$ e $y=y_{1} \mathbb{X}_{u}+y_{2} \mathbb{X}_{v}$, de modo que por linearidade;

$$
d N_{q}\left(d \mathbb{X}_{q}^{-1} x\right)=d N_{q}\left(x_{1}(1,0)+x_{2}(0,1)\right)=x_{1} N_{u}+x_{2} N_{v}
$$

Logo,

$$
\left\langle S_{q}(x), y\right\rangle=-x_{1} y_{1}\left\langle N_{u}, \mathbb{X}_{u}\right\rangle-x_{2} y_{2}\left\langle N_{v}, \mathbb{X}_{v}\right\rangle-x_{1} y_{2}\left\langle N_{u}, \mathbb{X}_{v}\right\rangle-y_{2} x_{1}\left\langle N_{v}, \mathbb{X}_{u}\right\rangle
$$

Por outro lado, como $\left\langle N, \mathbb{X}_{u}\right\rangle=0$ segue por diferenciação que $\left\langle N_{v}, \mathbb{X}_{u}\right\rangle=-\left\langle N, \mathbb{X}_{u v}\right\rangle$. Analogamente $\left\langle N_{u}, \mathbb{X}_{v}\right\rangle=-\left\langle N, \mathbb{X}_{v u}\right\rangle$. Como $\mathbb{X}$ diferenciável, vale que $\mathbb{X}_{u v}=\mathbb{X}_{v u}$, de modo que expandindo $\left\langle S_{q}(y), x\right\rangle$ e usando as identidades obtidas podemos concluir que $S_{q}$ é um operador auto-adjunto em relação ao produto interno $\langle$,$\rangle .$

Dada a forma auto-adjunta $S_{q}$ podemos associar uma forma uma forma bilinear simétrica em $T_{q} \mathbb{X}$, digamos $b$, pela regra;

$$
b: T_{q} \mathbb{X} \times T_{q} \mathbb{X} \rightarrow \mathbb{R} ; \quad(v, w) \mapsto b(v, w)=\left\langle S_{q}(v), w\right\rangle
$$

Do mesmo modo que a forma bilinear simétrica $g$ deu origem a forma quadrática $I, b$ também dará origem a uma forma quadrática. 
Definição 1.22. Seja $\mathbb{X}$ uma superfície parametrizada regular em $\mathbb{L}^{3}$ com $S_{q}$ o operador Weingarten. Então, a forma quadrática, induzida pelo operador de Weingarten;

$$
I I_{q}: T_{q} \mathbb{X} \rightarrow \mathbb{R} ; \quad w \mapsto I I_{q}(w)=\left\langle S_{q}(w), w\right\rangle
$$

é dita segunda forma fundamental de $\mathbb{X}$ em $q \in U$.

Definimos a curvatura Gaussiana e a curva média da superfície.

Definição 1.23. Sejam $\mathbb{X}: U \subset \mathbb{R}^{2} \rightarrow \mathbb{L}^{3}$ uma superfície parametrizada regular no espaço de Minkowski e $S_{q}$ o operador de Weingarten da superfície no ponto q. Definimos a Curvatura Gaussiana, $K$, e a Curvatura Média, $H$, da superfície $\mathbb{X}$ no ponto $q$ respectivamente por;

$$
\begin{array}{r}
K(q)=\epsilon \operatorname{det}\left(S_{q}\right) ; \\
H(q)=\epsilon \frac{1}{2} \operatorname{traço}\left(S_{q}\right)
\end{array}
$$

em que $\epsilon=-1$ se a superfície é tipo-espaço em $q$ e $\epsilon=1$ se a superfície é tipo-tempo.

Assim como fizemos para primeira forma fundamental, podemos expressar a segunda forma fundamental em termos da base coordenada. Os coeficientes são ditos coeficientes da segunda formal fundamental e denotados por;

$$
\begin{aligned}
e & =\left\langle S_{q}\left(\mathbb{X}_{u}\right), \mathbb{X}_{u}\right\rangle=\left\langle-N_{u}, \mathbb{X}_{u}\right\rangle=\left\langle N, \mathbb{X}_{u u}\right\rangle \\
f & =\left\langle S_{q}\left(\mathbb{X}_{u}\right), \mathbb{X}_{v}\right\rangle=\left\langle-N_{u}, \mathbb{X}_{v}\right\rangle=\left\langle-N_{v}, \mathbb{X}_{u}\right\rangle=\left\langle N, \mathbb{X}_{u v}\right\rangle \\
g & =\left\langle S_{q}\left(\mathbb{X}_{v}\right), \mathbb{X}_{v}\right\rangle=\left\langle-N_{v}, \mathbb{X}_{v}\right\rangle=\left\langle N, \mathbb{X}_{v v}\right\rangle
\end{aligned}
$$

Seja $B: T_{q} \mathbb{X} \times T_{q} \mathbb{X} \rightarrow \mathbb{R}$ a forma bilinear associada ao operador de Weingarten isto é, $B(u, v)=\left\langle S_{q}(u), v\right\rangle$. Com a expressão dos coeficientes da segunda forma fundamental podemos expressar a matriz representativa de $B$ na base coordenada,

$$
B \doteq\left(\begin{array}{cc}
e & f \\
f & g
\end{array}\right)
$$

Assim,

$$
B(v, w)=v^{t} B w=v^{t}\left(\begin{array}{ll}
e & f \\
f & g
\end{array}\right) w
$$


Por outro lado, se $A \doteq S_{q}$ denota a matriz representativa da transformação linear $S_{q}$ em relação a base coordenada, temos que,

$$
\left\langle v, S_{q}(w)\right\rangle=v^{t}\left(g_{i j}\right) A w=v^{t}\left(\begin{array}{cc}
E & F \\
F & G
\end{array}\right) A w .
$$

Consequentemente se $\mathbb{X}$ é não-degenerada decorre das Equações 1.11 e 1.12 que

$$
S_{q} \doteq A=\left(\begin{array}{cc}
E & F \\
F & G
\end{array}\right)^{-1}\left(\begin{array}{ll}
e & f \\
f & g
\end{array}\right) .
$$

De modo que podemos expressar as curvaturas Gaussiana e média de superfícies no espaço de Minkowski em termos dos coeficientes da primeira e segunda formas fundamentais. Seguindo a Definição 1.23 temos

$$
\begin{aligned}
K & =\epsilon \frac{e g-f^{2}}{E G-F^{2}} \\
H & =\epsilon \frac{1}{2} \frac{e G-2 f F+g E}{E G-F^{2}}
\end{aligned}
$$

Estas duas últimas equações nos dão uma forma prática para calcular as curvaturas Gaussiana e média de uma superfície conhecendo-se os coeficientes da primeira e segunda formas fundamentais.

A seguir discutiremos o conceito de curvaturas principais.

Lembramos que no espaço Euclidiano $\mathbb{E}^{3}$ existe o seguinte resultado, dado pelo Teorema da página 258 de [5], que nos permite definir os conceitos de curvaturas principais em superfícies no espaço Euclidiano;

Resultado 1.1. Sejam $V$ um espaço vetorial real de dimensão $2, A: V \rightarrow V$ uma aplicação linear auto-adujunta em relação ao produto interno Euclidiano $\langle,\rangle_{\mathbb{E}^{3}}$ (isto é $\langle A v, u\rangle_{\mathbb{E}^{3}}=$ $\langle v, A u\rangle_{\mathbb{E}^{3}}$ para todo $\left.u, v \in V\right)$ e $Q$ a forma quadrática positiva definida induzida por $A$ (isto $\left.e ́, Q(v)=\langle A v, v\rangle_{\mathbb{E}^{3}}\right)$. Então, existem uma base, de $V$, ortonormal $\left\{e_{1}, e_{2}\right\}$ de auto-vetores de A, tais que, a matriz de A expressa nesta é a matriz diagonal dada pelos auto-valores $\lambda_{1}$ e $\lambda_{2}$ de $e_{1}$ e $e_{2}$ respectivamente. Além disso, $\lambda_{1}$ e $\lambda_{2}$ são respectivamente o máximo e o mínimo da forma quadrática $Q$ restrita ao círculo unitário de $V$.

E sua demonstração utiliza essencialmente as hipóteses de $Q$ ser ser positiva definida, e que o círculo unitário em $V$, dados pelos vetores $u \in V$ tais que $\langle u, u\rangle_{\mathbb{E}^{3}}$ é um conjunto compacto. De modo que este teorema não se aplica para os espaços tangentes de superfícies 
no espaço de Minkowski, pois se $V$ for um subespaço vetorial de dimensão 2 tipo-tempo a métrica neste espaço tem índice 1 e o conjunto dos vetores unitários não é compacto.

Entretanto, nos casos em que o Operador de Weingarten é diagonalizável, podemos retomar com parte dos conceitos de curvaturas principais. Comecemos com a seguinte definiçã ${ }^{2}$, Definição 1.24. Seja $\mathbb{X}: U \subset \mathbb{R}^{2} \rightarrow \mathbb{E}^{3}$ uma superfície parametrizada regular não-degenerada no espaço de Minkowski. Diremos que $\mathbb{X}$ é Weingarten-diagonalizável em $q \in U$ desde que o operador de Weingarten $S_{q}: T_{q} \mathbb{X} \rightarrow T_{q} \mathbb{X}$ seja diagonalizável. Diremos que $X$ é uma superfície Weingarten-diagonalizável desde que seja Weingarten-diagonalizável em cada $q \in U$.

Com isso, vemos que em geral para superfícies no espaço de Minkowski nem sempre existirá curvaturas principais (que em superfícies no espaço Euclidiano são dadas pelos autovalores do operador de Weingarten). Entretanto nos casos em que a superfície é Weingartendiagonalizável temos a seguinte definição.

Definição 1.25. Seja $\mathbb{X}: U \subset \mathbb{R}^{2} \rightarrow \mathbb{L}^{3}$ uma superfície parametrizada regular não-degenerada em $\mathbb{L}^{3}$. Suponha que em $q \in U, \mathbb{X}$ seja Weingarten-diagonalizável. Então dizemos que os auto-valores de $S_{q}, k_{1}(q)$ e $k_{2}(q)$, são as curvaturas principais da superfície $\mathbb{X}$ em $q \in U$.

A Proposição a seguir, mostra que nos pontos onde a superfície é Weingarten-diagonalizável podemos escrever as curvaturas média e Gaussiana em termos das curvaturas principais.

Proposição 1.11. Sejam $\mathbb{X}: U \subset \mathbb{R}^{2} \rightarrow \mathbb{L}^{3}$ uma superfície parametrizada regular nãodegenerada no espaço de Minkowski e $q \in U$ tal que $S_{q}$ é diagonalizável. Então a curvatura média $H$ e a curvatura Gaussiana $K$ de $\mathbb{X}$ no ponto $q \in U$ são expressas por;

$$
\begin{array}{r}
K(q)=\epsilon k_{1}(q) k_{2}(q), \\
H(q)=\epsilon \frac{k_{1}(q)+k_{2}(q)}{2},
\end{array}
$$

em que, $k_{1}(q), k_{2}(q)$ são as curvaturas principais de $\mathbb{X}$ em $q \in U$ e $\epsilon=\langle N(q), N(q)\rangle$.

Demonstração: A demonstração segue diretamente das definições de curvatura média e de curvatura Gaussiana. De fato, supondo que $S_{q}$ é diagonálizel, temos que na base dos auto-vetores, a expressão matricial de $S_{q}$ será;

$$
S_{q} \doteq\left(\begin{array}{rr}
k_{1} & 0 \\
0 & k_{2}(q)
\end{array}\right) .
$$

\footnotetext{
${ }^{2}$ Formalmente esta seria uma convenção ou mesmo uma notação, visto que esta nomenclatura não é usual na literatura.
} 
E pela Definição 1.23 ,

$$
H(q)=\epsilon \frac{1}{2} \operatorname{traço}\left(\begin{array}{rr}
k_{1}(q) & 0 \\
0 & k_{2}(q)
\end{array}\right)=\epsilon \frac{k_{1}(q)+k_{2}(q)}{2} .
$$

Agora, pela Definição (1.23),

$$
K(q)=\epsilon \operatorname{det}\left(\begin{array}{rr}
k_{1}(q) & 0 \\
0 & k_{2}(q)
\end{array}\right)=\epsilon k_{1}(q) k_{2}(q) .
$$

E assim temos as expressões desejadas.

Vamos trazer, agora, noção de pontos umbílicos para as superfícies no espaço de Minkowski.

Lembremos que, em superfícies no espaço Euclidiano, pontos umbílicos são aqueles em que as curvaturas principais coincidem. Como em geral não definimos curvatura principais, não podemos falar da noção de pontos umbílicos via curvaturas principais. Deste modo, temos uma noção um pouco mais geral de pontos umbílicos, para superfícies não-degeneradas.

Definição 1.26. Sejam $\mathbb{X}: U \subset \mathbb{R}^{2} \rightarrow \mathbb{L}^{3}$ uma superfície parametrizada regular nãodegenerada no espaço de Minkowski e $q \in U$. Então $q$ é dito um Ponto umbílico de $\mathbb{X}$ desde que, existe um número real $\lambda(q)$ tal que

$$
\left\langle S_{q}(v), w\right\rangle=\lambda(q)\langle u, v\rangle ; \quad \forall v, w \in T_{q} \mathbb{X}
$$

isto é, as formas bilineares simétricas b e g são proporcionais no ponto q. Além disso, dizemos que a superfície $\mathbb{X}$ é uma Superfície umbílica (ou totalmente umbílica) desde que seja umbílica em cada $q \in U$.

Notamos que se $\mathbb{X}$ é superfície não-degenerada e umbílica a associação $q \mapsto \lambda(q)$ depende diferencialmente de $q$, isto é, a função $\lambda$ é diferenciável em $U$.

Teorema 1.3. Seja $\mathbb{X}: U \subset \mathbb{R}^{2} \rightarrow \mathbb{L}^{3}$ uma superfície parametrizada regular não-degenerada. Suponha que a superfície é umbílica no ponto $q \in U$, então a superfície é Weingartendiagonalizável em $q$.

Demonstração: Como $\mathbb{X}$ é uma superfície não-degenerada existe uma base ortogonal $\left\{\alpha_{1}, \alpha_{2}\right\}$ de $T_{q} \mathbb{X}$. Supondo agora que $\mathbb{X}$ é umbílica em $q$, assim existe um número real $\lambda$ tal que

$$
\left\langle S_{q}(v), w\right\rangle=\lambda\langle v, w\rangle, \quad \forall v, w \in T_{q} \mathbb{X} .
$$


Em particular $\left\langle S_{q}\left(\alpha_{1}\right), \alpha_{2}\right\rangle=\lambda\left\langle\alpha_{1}, \alpha_{2}\right\rangle=0$. De modo que temos duas opções: $S_{q}\left(\alpha_{1}\right)$ é nulo ou $S_{q}\left(\alpha_{1}\right)$ é paralelo a $\alpha_{1}$.

Caso 1 Suponha que $S_{q}\left(\alpha_{1}\right)=0$. Pela relação 1.18 temos que

$$
0=\left\langle S_{q}\left(\alpha_{1}\right), \alpha_{1}\right\rangle=\lambda\left\langle\alpha_{1}, \alpha_{1}\right\rangle,
$$

o que acarreta em $\lambda=0$. Logo $S_{q}\left(\alpha_{1}\right)=0=\lambda \alpha_{1}$ e $\lambda$ é então um auto-valor do operador de Weingarten.

Caso 2 Suponha que $S_{q}\left(\alpha_{1}\right)=r \alpha_{1}$, para algum $r \neq 0$ número real. Decorre da Equação (1.18 que

$$
\left\langle r \alpha_{1}, \alpha_{1}\right\rangle\left\langle S_{q}\left(\alpha_{1}\right), \alpha_{1}\right\rangle=\lambda\left\langle\alpha_{1}, \alpha_{1}\right\rangle
$$

Donde concluímos que $r=\lambda$ de modo que $\lambda$ é um auto-valor do operador Weingarten.

Nos dois casos concluímos que o número $\lambda$, dado na hipótese de $q$ ser ponto umbílico, é um auto-valor do operador de Weingarten com auto-vetor $\alpha_{1}$. Simetricamente, repetindo este argumento para $S_{q}\left(\alpha_{2}\right)$ concluímos que $S_{q}$ admite uma base de auto-vetores na qual a diagonal é dada pelo valor $\lambda$. Consequentemente o operador de Weingarten é diagonalizável e por definição isto implica que $\mathbb{X}$ é Weingarten-diagonalizável em $q$.

Como uma consequência deste Teorema temos que a Definição $(1.1 .4)$ de ponto umbílico é de fato mais geral que a definição via curvaturas principais.

Corolário 1.3. Seja $\mathbb{X}$ uma superfície parametrizada regular não-degenerada no espaço de Minkowski. Suponha que $\mathbb{X}$ é Weingarten-diagonalizável em $q$, então $\mathbb{X}$ é umbílica q se, e somente se, $k_{1}(q)=k_{2}(q)$.

Demonstração: De fato, supondo $\mathbb{X}$ Weingarten-diagonalizável em $q$ temos que existem as curvaturas principais $k_{1}$ e $k_{2}$ no ponto $q$ e pelo Teorema (1.3) garantimos que estas curvaturas coincidem com o valor comum $\lambda$. A recíproca é dada verificando que a Equação (1.17) é verdadeira para os vetores da base ortonogal de auto-vetores e então, por bilinearidade, será verdadeira para qualquer par de vetores no plano tangente.

Com as noções de curvaturas principais e pontos umbílicos para superfícies Weingartendiagonalizáveis, podemos demonstrar o seguinte resultado;

Teorema 1.4. Seja $\mathbb{X}$ uma superfície parametrizada regular não-degenerada no espaço de Minkowski. Suponha que $\mathbb{X}$ é Wengarten-diagonalizável em $q \in U$, então;

$$
H^{2}(q)-\epsilon K(q) \geq 0
$$


Além disso, a igualdade ocorre se, e somente se, q é um ponto umbílico de $\mathbb{X}$.

Demonstração: De fato, se $k_{1}$ e $k_{2}$ são as curvaturas principais de $\mathbb{X}$ no ponto $q$;

$$
0 \leqslant\left(\frac{k_{1}-k_{2}}{2}\right)^{2}=\left(\frac{k_{1}+k_{2}}{2}\right)^{2}-k_{1} k_{2}=H^{2}-\epsilon K,
$$

em que $H$ e $K$ são dadas pelas Equações 1.15 e 1.16 respectivamente. Agora a igualdade ocorre se, e somente se, $k_{1}=k_{2}$ e sendo por hipótese $\mathbb{X}$ Weingarten-diagonalizável, decorre do Corolário 1.3 que isto equivale a $\mathbb{X}$ ser úmblica no ponto $q$.

A diagonalização do operador de Weingarten depende da existência de raízes do polinômio característico. Denotando por $A=\left(a_{i j}\right)$ a matriz representativa de $S_{q}$ em alguma base o polinômio característico $P(\lambda)$ é dado por;

$$
P(\lambda)=\operatorname{det}(A-\lambda I)=\lambda^{2}-\lambda\left(a_{11}+a_{22}\right)+a_{11} a_{22}-a_{12} a_{21} .
$$

Daí segue que a equação do polinômio característico é expressa por

$$
P(\lambda)=\lambda^{2}-2 \epsilon H \lambda+\epsilon K
$$

cujo discriminante é;

$$
\triangle=4\left(H^{2}-\epsilon K\right)
$$

Deste modo, podemos caracterizar as superfícies Weingarten-diagonalizáveis em termos do sinal do discriminante.

$\triangle>0$ Neste caso a superfície é Weingarten-diagonalizável, seja ela tipo-espaço ou tipo-tempo.

$\triangle<0$ Neste caso a superfície não é Weingarten-dagonalizável. Recorde que pelo Resultado (1.1) a superfície deverá ser tipo-tempo, e juntamente com o Teorema (1.4 podemos garantir que a superfície não tem pontos umbílicos.

$\triangle=0$ Inconclusivo. Entretanto se a superfície é tipo-espaço pelo Resultado 1.1 garantimos que é Weingarten-diagonalizável e pelo Teorema (1.4) garantimos que é umbílica com a curvatura principal dada por $k=-H$.

O seguinte resultado nos dá uma espécie de classificação de todas as superfícies umbílicas no espaço de Minkowski. 
Teorema 1.5. Seja $\mathbb{X}$ uma superfície parametrizada regular não-degenerada no espaço de Minkowski. Suponha que $\mathbb{X}$ é uma superfície umbílica, então a superfície está contida em um plano, plano hiperbólico ou em uma pseudo-esfera, sendo que os dois últimos casos ocorrem somente se a superfície é respectivamente tipo-espaço ou tipo-tempo.

Demonstração: Suponha que $\mathbb{X}$ é totalmente umbílica. Pelo Teorema (1.3) garantimos que em cada $q \in U, \mathbb{X}$ é Weingaten-diagonalizável e pelo seu Corolário (1.3), para cada $w \in T_{q} \mathbb{X}$ verificamos que existem uma função diferenciável $\lambda(q)$ tal que

$$
d N_{q}(w)=\lambda(q) w
$$

Na base coordenada, com $w=a \mathbb{X}_{u}+b \mathbb{X}_{v}$, temos

$$
a N_{u}(q)+b N_{v}(q)=\lambda(q) a \mathbb{X}_{u}+\lambda b \mathbb{X}_{v}
$$

Como esta relação vale para qualquer $w$ no plano tangente, concluímos que

$$
\begin{aligned}
N_{u}(q)=\lambda(q) \mathbb{X}_{u}(q), & N_{u v}=\lambda_{v} \mathbb{X}_{u}+\lambda \mathbb{X}_{u v} \\
N_{v}(q)=\lambda(q) \mathbb{X}_{v}(q), & N_{v u}=\lambda_{u} \mathbb{X}_{v}+\lambda \mathbb{X}_{v u}
\end{aligned}
$$

Em que diferenciamos em relação a $v$ e a $u$ respectivamente. Subtraindo estas relações e usando que $\mathbb{X}_{u v}=\mathbb{X}_{v u}$ e $N_{u v}=N_{v u}$, temos

$$
\lambda_{v} \mathbb{X}_{u}-\lambda_{u} \mathbb{X}_{v}=0
$$

Por independência linear dos vetores coordenados, devemos ter que $\lambda_{u}(q)=\lambda_{v}(q)=0$, e sendo $U$ conexo isto implica que $\lambda(q)$ é uma função constante. Assim temos dois possíveis casos;

Se $\lambda=0$ : temos pelas relações 1.23 e 1.24 que $N_{u}=N_{v}=0$ e novamente por conexidade de $U$ o vetor $N$ é constante e concluímos que $\mathbb{X}$ está contida em um plano (note que $\mathbb{X}$, neste caso pode ser tanto tipo-espaço quanto tipo-tempo).

Se $\lambda \neq 0$ : definimos uma função $p$;

$$
p: U \subset \mathbb{R}^{2} \rightarrow \mathbb{L}^{3} ; \quad(u, v) \mapsto p(u, v)=\mathbb{X}(u, v)-\frac{1}{\lambda} N(u, v) \in \mathbb{L}^{3},
$$

que varia diferenciavelmente em $U$. Diferenciando $p$ em relação a $u$ e $v$ e usando as expressões (1.23) e 1.24), garantimos que $p_{u}=p_{v}=0$. Por conexidade de $U$ temos que $p(u, v)=p_{0}$ é 
constante. Deste modo, $\mathbb{X}-p_{0}=\frac{1}{\lambda} N$ em todos os pontos de $U$, e assim

$$
\left\langle\mathbb{X}-p_{0}, \mathbb{X}-p_{0}\right\rangle=\frac{1}{\lambda^{2}}\langle N, N\rangle=\frac{\epsilon}{\lambda^{2}},
$$

em que $\epsilon=-1$ se superfície é tipo-espaço e $\epsilon=+1$ se a superfície é tipo-tempo. No primeiro caso a superfície estará contida do plano hiperbólico $\mathbb{H}^{2}\left(p_{0} ; \lambda\right)$ que passa por $p_{0}$ e tem raio $\lambda$ e no segundo caso a superfície está contida na pseudo-esfera $\mathbb{S}^{2}\left(p_{0} ; \lambda\right)$ que tem origem em $p_{0}$ e raio $\lambda$. 


\section{Capítulo 2}

\section{Formas Diferenciais e o Método do Referencial Móvel}

Neste capítulo introduziremos o Método do referencial móvel. Este é um método desenvolvido no século passado, principalmente, pelo matemático francês Élie Joseph Cartan (1869-1951), e atualmente compõe uma das abordagens clássicas da Geometria Diferencial. Esta abordagem depende essencialmente do conceito de Formas Diferenciais, a qual dedicaremos uma introdução na próxima. Em seguida introduziremos o conceito de Método do Referencial Móvel e aplicaremos em duas situações; em superfícies no espaço Euclidiano e superfícies no espaço de Minkowski.

Este capítulo é essencial para a leitura do restante do texto, pois, conforme veremos as Transformações de Bäcklund e suas aplicações serão dadas de acordo com o Método do Referencial Móvel.

\subsection{Formas Diferenciais}

Nesta seção, baseada no livro texto [20], introduziremos conceitos de formas diferenciais, produto tensorial, produto externo e derivada exterior de formas diferenciais e suas principais propriedades. Todos estes conceitos não são métricos, de modo que não há necessidade de adaptar nenhuma definição para o contexto do espaço de Minkowski.

Seja $\mathbb{R}^{n}$ espaço vetorial sobre o corpo dos reais. Definimos o espaço dual de $\mathbb{R}^{n}$ pelo espaço das transformações lineares de $\mathbb{R}^{n}$ em $\mathbb{R}$, o denotaremos por $\left(\mathbb{R}^{n}\right)^{*}=\left\{L: \mathbb{R}^{n} \rightarrow \mathbb{R} \mid L\right.$ é linear $\}$. Podemos verificar que o espaço dual $\left(\mathbb{R}^{n}\right)^{*}$ é um espaço vetorial real com as operações; $(T+L)(a)=T(a)+L(a)$ e $(\mu T)(a)=\mu T(a)$ para $L, T \in\left(\mathbb{R}^{n}\right)^{*}$ e $\mu \in \mathbb{R}$. 
Lema 2.1. Seja $B=\left\{x_{1}, \ldots, x_{n}\right\}$ uma base para $\mathbb{R}^{n}$. Então o conjunto das aplicações

$$
d x_{j}: \mathbb{R}^{n} \rightarrow \mathbb{R} \quad ; \quad v=\sum_{i=1}^{n} v_{i} x_{i} \mapsto d x_{j}(v)=v_{j} \in \mathbb{R},
$$

formam uma base de $\left(\mathbb{R}^{n}\right)^{*}$, dita base dual associada a base $B$.

Demonstração: De fato, primeiramente, para cada $j \in\{1, \ldots, n\} d x_{j} \in\left(\mathbb{R}^{n}\right)^{*} ;$ sejam $u, v \in$ $\mathbb{R}^{n}$ e $\lambda \in \mathbb{R}$. Assim

$$
\begin{aligned}
d x_{j}(u+\lambda v) & =d x_{j}\left(\sum_{i=1}^{n}\left(u_{i}+\lambda v_{i}\right) x_{i}\right)=u_{j}+\lambda v_{j}= \\
& =d x_{j}\left(\sum_{i=1}^{n}\left(u_{i}\right)\right)+\lambda d x_{j}\left(\sum_{i=1}^{n}\left(v_{i}\right)\right)=d x_{j}(u)+\lambda d x_{j}(v)
\end{aligned}
$$

Observe que as transformações lineares $d x_{j}$ geram $\left(\mathbb{R}^{n}\right)^{*}$ quer dizer que para toda $T \in\left(\mathbb{R}^{n}\right)^{*}$ devem existir $\mu_{1}, \ldots \mu_{n}$ escalares em $\mathbb{R}$ tais que $T=\sum_{j=1}^{n} \mu_{j} d x_{j}$. O que sempre podemos garantir, pois dado $T$ tome $\mu_{i}=T\left(x_{i}\right)$. Assim teremos

$$
T(v)=T\left(\sum_{i=1}^{n} v_{i} x_{i}\right)=\sum_{i=1}^{n} v_{i} T\left(x_{i}\right)=\sum_{i=1}^{n} v_{i} \mu_{i}=\sum_{i=1}^{n} \mu_{i} d x_{i}(v),
$$

que vale para todo $v$. Logo $T=\sum_{i=1}^{n} \mu_{i} d x_{i}$.

Por último devemos mostrar que $\left\{d x_{1}, \ldots, d x_{n}\right\} \in\left(\mathbb{R}^{n}\right)^{*}$ é um conjunto linearmente independente. Suponhamos que $\sum_{i=1}^{n} \mu_{i} d x_{i}(v)=0$ para todo $v \in \mathbb{R}^{n}$. Assim tomemos $v=1 x_{j}$ para cada $j$ de modo que

$$
0=\sum_{i=1}^{n} \mu_{i} d x_{i}\left(1 x_{j}\right)=\mu_{1} 0+\ldots+\mu_{j} 1+\ldots+\mu_{n} 0=\mu_{j} .
$$

Assim concluímos que este conjunto é de fato linearmente independente. E demonstramos este Lema.

Deste modo se $f: U \subset \mathbb{R}^{n} \rightarrow \mathbb{R}$ é uma função diferenciável em $a \in U$ sua diferencial em $a$ é uma transformação linear $d f_{a}: \mathbb{R}^{n} \rightarrow \mathbb{R}$ dada pela derivada direcional em $a$, isto é,

$$
d f_{a}(v)=D_{v} f(a)=\lim _{t \rightarrow 0} \frac{f(a+t v)-f(a)}{t} .
$$

Conforme a notação do Lema (2.1), com $B=\left\{e_{1}, \ldots, e_{n}\right\}$ base canônica de $\mathbb{R}^{n}$, temos que a transformação linear $d f_{a}$ expressa na base dual, é dada por $d f_{a}=\sum_{i=1}^{n} \mu_{i} d x_{i}$ em que

$$
\begin{aligned}
\mu_{j} & =d f_{a}\left(e_{j}\right)=D_{e_{j}} f(a)=\lim _{t \rightarrow 0} \frac{f\left(a+t e_{j}\right)+f(a)}{t} \\
& =\lim _{t \rightarrow 0} \frac{f\left(a_{1}, \ldots, a_{j}+t e_{j}, \ldots, a_{n}\right)+f\left(a_{1}, \ldots, a_{n}\right)}{t}=\frac{\partial f}{\partial x_{j}}(a) .
\end{aligned}
$$


Ou seja a diferencial de $f$ no ponto $a$ assume a forma

$$
d f_{a}=\sum_{i=1}^{n} \frac{\partial f}{\partial x_{j}}(a) d x_{i} .
$$

Esta expressão entre elementos do espaço dual motiva a seguinte definição.

Definição 2.1. (1-Formas diferenciais)Dizemos que $\omega$ é uma forma diferencial de grau 1, ou uma 1-forma diferencial, em um aberto $U \subset \mathbb{R}^{n}$ desde que seja uma associação que a cada ponto $p \in U$ corresponde a um $\omega_{p}$ elemento do espaço dual de $\mathbb{R}^{n}$, tal que, quando expresso em alguma base $\left\{d x_{1}, \ldots, d x_{n}\right\}, \omega_{p}=\sum_{i=1}^{n} a_{i}(p) d x_{i}$ as funções $a_{i}: U \subset \mathbb{R}^{n} \rightarrow \mathbb{R}$ são diferenciáveis.

Naturalmente a diferencial de uma função, $f: U \subset \mathbb{R}^{n} \rightarrow \mathbb{R}$ diferenciável, em qualquer ponto $a \in U$ é uma 1-forma diferencial.

Observação 2.1. Sendo as projeções $\pi_{j}: \mathbb{R}^{n} \rightarrow \mathbb{R}$ funções diferenciáveis, temos que em cada $p \in \mathbb{R}^{n}$, sua diferencial é uma 1-forma diferenciál e ainda mais, note que em cada $p \in \mathbb{R}^{n}$ o conjunto $\left\{d\left(\pi_{1}\right)_{p}, \ldots, d\left(\pi_{j}\right)_{p}\right\}$ forma uma base do espaço dual associada a base canônica de $\mathbb{R}^{n}$.

De agora em diante nos restringiremos as 1-formas diferenciais em abertos $U \subset \mathbb{R}^{2}$. Ainda denotaremos as projeções $\pi_{1}$ e $\pi_{2}$ por respectivamente $u$ e $v$, de modo que para cada $q \in U$, $\left\{d u_{q}, d v_{q}\right\}$ será uma base de $\left(\mathbb{R}^{2}\right)^{*}$ associada a base canônica $\left\{e_{1}=(1,0), e_{2}=(0,1)\right\}$. Além disso, se $\omega$ é uma 1-forma diferencial em $U \in \mathbb{R}^{2}$ escreveremos

$$
\omega_{q}=P(q) d u_{q}+Q(q) d v_{q}, \quad \in\left(\mathbb{R}^{2}\right)^{*} .
$$

Introduziremos de maneira natural, a operação de soma de 1-formas diferenciais e multiplicação por funções reais diferenciáveis.

Definição 2.2. Sejam $\omega$ e $\bar{\omega}$ 1-formas diferenciais e $f$ uma função real diferenciável em $U \subset \mathbb{R}^{2}$. Definimos a soma de $\omega$ e $\bar{\omega}$ como sendo a 1-forma diferencial dada por

$$
(\omega+\bar{\omega})_{q}=\omega_{q}+\bar{\omega}_{q}
$$

E a multiplicação de $\omega$ por $f$ como sendo a 1-forma diferencial dada por

$$
(f \omega)_{q}=f(q) \omega_{q}
$$


Assim fica definida a expressarão $\omega=P d u+Q d v$. Além disso se $\omega=P d u+Q d v$, $\bar{\omega}=\bar{P} d u+\bar{Q} d v$ e $f$ uma função real diferenciável. Segue que

$$
\omega+f \bar{\omega}=(P+f \bar{P}) d u+(Q+f \bar{Q}) d v
$$

Definição 2.3. Dadas duas 1-formas diferenciais em $U \subset \mathbb{R}^{2}, \omega$ e $\bar{\omega}$ dizemos que elas são linearmente independentes desde que, para cada $q \in U, \omega_{q}$ e $\bar{\omega}_{q}$ são linearmente independentes como vetores de $\left(\mathbb{R}^{2}\right)^{*}$.

Observação 2.2. Sabendo que $d u$ e $d v$ formam uma base temos que $\omega=P d u+Q d v$ e $\bar{\omega}=\bar{P} d u+\bar{Q} d v$ são linearmente independentes se, e somente, se para cada $q \in U \subset \mathbb{R}^{2}$

$$
\left|\begin{array}{ll}
P(q) & Q(q) \\
\bar{P}(q) & \bar{Q}(q)
\end{array}\right| \neq 0 .
$$

Definição 2.4. (Produto Tensorial) Seja duas 1-formas diferenciais em $U \subset \mathbb{R}^{2}$, $\omega$ e $\bar{\omega}$, definimos o produto tensorial destes elementos pela associação que corresponde a cada $q \in U$ a aplicação bilinear dada por;

$$
(\omega \otimes \bar{\omega})_{q}: \mathbb{R}^{2} \times \mathbb{R}^{2} \rightarrow \mathbb{R} \quad ; \quad V, W \mapsto(\omega \otimes \bar{\omega})_{q}(V, W)=\omega(V)_{q} \bar{\omega}_{q}(\omega)
$$

Observemos que o produto $\omega \otimes \bar{\omega}$ não é comutativo e a aplicação bilinear $(\omega \otimes \bar{\omega})_{q}$ não é simétrica. De fato basta considerarmos os exemplos; $(d u \otimes d v)_{q}\left(e_{1}, e_{2}\right)=1$ e $(d v \otimes$ $d u)_{q}\left(e_{1}, e_{2}\right)=0$, bem como $(d u \otimes d v)_{q}\left(e_{2}, e_{1}\right)=0$.

Como o produto tensorial $\omega \otimes \bar{\omega}$ é uma aplicação bilinear, fica definida a soma entre $\omega \otimes \bar{\omega}$ e $\tilde{\omega} \otimes \hat{\omega}$ como a soma natural entre aplicações bilineares.

Proposição 2.1. (Propriedades do Produto Tensorial) Sejam $\omega, \bar{\omega}$ e $\hat{\omega} 1$-formas diferenciais em $U \subset \mathbb{R}^{2}$ e $f: U \subset \mathbb{R}^{2} \rightarrow \mathbb{R}$ uma função real diferenciável em $U$. Então

1. $(\omega+\bar{\omega}) \otimes \hat{\omega}=\omega \otimes \hat{\omega}+\bar{\omega} \otimes \hat{\omega}$,

2. $\hat{\omega} \otimes(\omega+\bar{\omega})=\hat{\omega} \otimes \omega+\hat{\omega} \otimes \bar{\omega}$,

3. $(f \omega) \otimes \bar{\omega}=\omega \otimes(f \bar{\omega})=f(\omega \otimes \bar{\omega})$,

4. Se $\omega=P d u+Q d v$ e $\bar{\omega}=\bar{P} d u+\bar{Q} d v$, então

$$
\omega \otimes \bar{\omega}=P \bar{P} d u \otimes d u+P \bar{Q} d u \otimes d v+\bar{P} Q d v \otimes d u+Q \bar{Q} d v \otimes d v
$$

\section{Demonstração:}


1. Para cada $q \in U$ e para cada $V, W \in \mathbb{R}^{2}$

$$
\begin{aligned}
((\omega+\bar{\omega}) \otimes \hat{\omega})_{q}(V, W) & =(\omega+\bar{\omega})_{q}(V, W) \hat{\omega}_{q}(V, W) \\
& =\omega_{q}(V, W) \hat{\omega}_{q}(V, W)+\bar{\omega}_{q}(V, W) \hat{\omega}_{q}(V, W) \\
& =(\omega \otimes \hat{\omega})_{q}(V, W)+(\bar{\omega} \otimes \hat{\omega})_{q}(V, W) .
\end{aligned}
$$

2. Análogo ao item anterior.

3. Basta notar que para cada $q \in U$ e para cada $V, W \in \mathbb{R}^{2}$ temos

$$
[f(\omega \otimes \bar{\omega})]_{q}(V, W)=f(q)\left(\omega_{q}(V) \bar{w}_{q}(W)\right)=f(q) \omega_{q}(V) \bar{w}_{q}(W)
$$

Assim o resultado segue pela comutatividade dos números reais e pela definição de produto tensorial.

4. Bastar realizar o produto $(P d u+Q d v) \otimes(\bar{P} d u+\bar{Q} d v)$ utilizando 1,2 e 3 .

Definiremos também o produto exterior entre duas 1-formas diferenciais.

Definição 2.5. (Produto Exterior) Sejam $\omega$ e $\bar{\omega} 1$-formas diferenciais em $U \subset \mathbb{R}^{2}$. Definimos o produto exterior entre $\omega$ e $\bar{\omega}$ pela associação que a cada $q \in U$ corresponde a uma aplicação dada por

$$
(\omega \wedge \bar{\omega})_{q}: \mathbb{R}^{2} \times \mathbb{R}^{2} \rightarrow \mathbb{R} ; \quad(V, W) \mapsto(\omega \otimes \bar{\omega})_{q}(V, W)-(\bar{\omega} \otimes \omega)_{q}(V, W)
$$

Note que o produto exterior como definido é uma forma bilinear e anti-simétrica. De fato, note que para todo $V, W \in \mathbb{R}^{2}$,

$$
\begin{aligned}
(\omega \wedge \bar{\omega})_{q}(V, W) & =\omega_{q}(V) \bar{\omega}_{q}(W)-\bar{\omega}_{q}(V) w_{q}(W) \\
& =\left|\begin{array}{cc}
\omega_{q}(V) & \bar{\omega}_{q}(V) \\
\omega_{q}(W) & \bar{\omega}_{q}(W)
\end{array}\right|
\end{aligned}
$$

de modo que nossa afirmação decorre das propriedades do determinante (linear em cada linha e anti-simétrico pela troca de duas linhas).

Observação 2.3. A associação, pela qual definimos o produto exterior é anti-simétrica em relação a $\omega$ e $\bar{\omega}$, isto é, $\omega \wedge \bar{\omega}=-\bar{\omega} \wedge \omega$. Em particular temos que,

$$
d u \wedge d v=-d v \wedge d u \quad \text { e } \quad d u \wedge d u=d v \wedge d v=0 .
$$


Em geral, temos as seguintes propriedades para o produto exterior;

Proposição 2.2. (Propriedades do produto exterior) Sejam $\omega, \bar{\omega}$ e $\hat{\omega} 1$-formas diferenciais em $U \subset \mathbb{R}^{2}$ e $f: U \subset \mathbb{R}^{2}$ uma função real diferenciável em $U$, então

1. $\omega \wedge(\bar{\omega}+\hat{\omega})=\omega \wedge \bar{\omega}+\omega \wedge \hat{\omega}$

2. $(\bar{\omega}+\hat{\omega}) \wedge \omega=\bar{\omega} \wedge \omega+\hat{\omega} \wedge \omega$,

3. $(f \omega) \wedge \bar{\omega}=\omega \wedge(f \bar{\omega})=f(\omega \wedge \bar{\omega})$,

4. Se $\omega=P d u+Q d v$ e $\bar{\omega}=\bar{P} d u+\bar{Q} d v$, então

$$
\omega \wedge \bar{\omega}=(P \bar{Q}-Q \bar{P})(P \bar{Q}-Q \bar{P}) d u \wedge d v
$$

5. $\omega \wedge \bar{\omega}=-\bar{\omega} \wedge \omega$.

Demonstração: De fato, para os itens (1)-(3) basta usar a definição e as propriedades (1),(2) e (3) da Proposição 2.1). O item (5) se dá conforme comentado acima. Por último, (4) resultará das proriedades (1)-(3) e da Observação(2.3). De fato,

$$
\begin{aligned}
(P d u+Q d v) \wedge(\bar{P} d u+\bar{Q} d v)= & P \bar{P} d u \wedge d u+P \bar{Q} d u \wedge d v+ \\
& +Q \bar{P} d v \wedge d u+Q \bar{Q} d v \wedge d v \\
= & (P \bar{Q}-Q \bar{P}) d u \wedge d v
\end{aligned}
$$

Esta Proposição nos dá uma caracterização para independência linear entre formas diferenciais definida em (2.3). Conforme mostra o Corolário a seguir.

Corolário 2.1. Sejam $\omega$ e $\bar{\omega}$ 1-formas diferenciais em $U \subset \mathbb{R}^{2}$, então $\omega$ e $\bar{\omega}$ são linearmente independentes se, e somente se, para cada $q \in U,(\omega \wedge \bar{\omega})_{q} \not \equiv 0$.

Demonstração:De fato, sejam $\omega=P d u+Q d v$ e $\bar{\omega}=\bar{P} d u+\bar{Q} d v$, temos pelo item (4) da Proposição 2.2 que

$$
\omega \wedge \bar{\omega}=(P \bar{Q}-Q \bar{P})(P \bar{Q}-Q \bar{P}) d u \wedge d v=0
$$

equivale a

$$
P \bar{Q}-Q \bar{P}=0 .
$$


Assim, $\omega \wedge \bar{\omega}=0$ se, e somente se

$$
\left|\begin{array}{cc}
P(q) & Q(q) \\
\bar{P}(q) & \bar{Q}(q)
\end{array}\right|=0 .
$$

E assim o resultado segue pela definição (2.3).

Dadas duas 1-formas diferenciais, podemos notar que o produto exterior destas não é uma 1-forma, pois 1-formas diferenciais dão origem a transformações lineares enquanto produto exterior gera uma aplicação bilinear anti-simétrica. Entretanto o produto exterior pertence a uma outra classe, a saber, a classe das 2-formas diferenciais que definiremos a seguir.

Definição 2.6. (2-forma diferencial) Uma 2-forma diferencial em um aberto $U \subset \mathbb{R}^{2}$ é uma associação, $\phi$, de cada $q \in \mathbb{R}^{2}$ com uma transformação bilinear anti-simétrica;

$$
\phi_{q}: \mathbb{R}^{2} \times \mathbb{R}^{2} \rightarrow \mathbb{R} ; \quad(V, W) \mapsto \phi_{q}(V, W)=f(q)(d u \wedge d v)_{q}(V, W),
$$

em que $f: U \subset \mathbb{R}^{2} \rightarrow \mathbb{R}$ é uma função real diferenciável em $U$.

Observação 2.4. Alternativamente alguns autores definem 2-formas diferenciais com a condição de Alternada ao invés de Anti-Simétrica. Em geral, uma transformação bilinear $B: E \times E \rightarrow \mathbb{R}$, em $E$ espaço vetorial real, é dita Alternada, desde que, para cada $V \in E$ verifica-se que $B(V, V)=0$. Deste modo, note que pela relação;

$$
B(V+W, V+W)=B(V, V)+B(V, W)+B(W, V)+B(W, W)
$$

estes dois conceitos são equivalentes.

Definimos, naturalmente, a soma de duas 2-formas diferenciais, $\phi, \bar{\phi}$, e o produto por uma função real $h$ diferenciável em $U$ por

$$
\phi+h \bar{\phi}=(f+h \bar{f}) d u \wedge d v
$$

Temos também a definição de diferencial exterior de 1-forma diferencial;

Definição 2.7. (Diferencial Exterior) Seja $\omega=P d u+Q d v$ uma 1-forma diferencial em $U \subset \mathbb{R}^{2}$. Definimos a diferencial exterior de $\omega$ como sendo a 2-forma diferencial dada por

$$
d \omega=d P \wedge d u+d Q \wedge d v
$$

na qual $d P$ e $d Q$ são as respectivas 1-formas diferenciais em $U$ das funções $P$ e $Q$. 
Neste contexto, convém definir uma 0-forma diferencial em um aberto $U \subset \mathbb{R}^{2}$ pelas funções reais $f: U \subset \mathbb{R}^{2} \rightarrow \mathbb{R}$ diferenciáveis em $U$. De modo que a diferencial exterior de $\mathrm{f}$ é definida pela 1-forma diferencial em $U$, dada pela própria diferencial de $f$.

Proposição 2.3. (Propriedades da Diferencial Exterior) Sejam $\omega=P d u+Q d v$ e $\bar{\omega}=$ $\bar{P} d u+\bar{Q} d v$ 1-formas diferenciais em $U \subset \mathbb{R}^{2}$ e $f$ uma 0-forma diferencial em $U$, então;

1. $d \omega=\left(Q_{u}-P_{v}\right) d u \wedge d v$.

2. $d(d f) \equiv 0$

3. $d(\omega+\bar{\omega})=d \omega+d \bar{\omega}$

4. $d(f \omega)=d f \wedge \omega+f d \omega$

\section{Demonstração:}

1. De fato usando as propriedades da Proposição (2.2) e conforme a Observação (2.3), temos que

$$
\begin{aligned}
d \omega & =d P \wedge d u+d Q \wedge d v \\
& =\left(P_{u} d u+P_{v} d v\right) \wedge d u+\left(Q_{u} d u+Q_{v} d v\right) \wedge d v \\
& =P_{u} d u \wedge d u+P_{v} d v \wedge d u+Q_{u} d u \wedge d v+Q_{v} d v \wedge d v \\
& =-P_{v} d u \wedge d v+Q_{u} d u \wedge d v=\left(Q_{v}-P_{u}\right) d u \wedge d v
\end{aligned}
$$

2. Segue do Teorema de Cauchy para funções diferenciáveis (que nos garante que $f_{u v}=$ $\left.f_{v u}\right)$ e das propriedades dadas na Proposição 2.2 . De fato

$$
\begin{aligned}
d(d f) & =d\left(f_{u} d u+f_{v} d v\right)=d f_{u} \wedge d u+d f_{v} \wedge d v \\
& =\left(f_{u u} d u+f_{u v} d v\right) \wedge d u+\left(f_{v u} d u+f_{v v} d v\right) \wedge d v \\
& =f_{u v} d v \wedge d u+f_{v u} d u \wedge d v=\left(-f_{u v}+f_{v u}\right) d u \wedge d v \\
& =0
\end{aligned}
$$

3. É uma consequência das propriedades de produto externo dadas na Proposição 2.2 .

$$
\begin{aligned}
d(\omega+\bar{\omega}) & =d((P+\bar{P}) d u+(Q+\bar{Q}) d v) \\
& =d(P+\bar{P}) \wedge d u+d(Q+\bar{Q}) \wedge d v \\
& =d P \wedge d u+d \bar{P} \wedge d u+d Q \wedge d v+d \bar{Q} \wedge d v \\
& =d \omega+d \bar{\omega}
\end{aligned}
$$


4. Novamente, o resultado segue das propriedades da Proposição 2.2 , e também do item $(3)$.

$$
\begin{aligned}
d(f \omega) & =d(f P d u+f Q d v)=d(f P) \wedge d u+d(f Q) \wedge d v \\
& =P d f \wedge d u+f d P \wedge d u+Q d f \wedge d v+f d Q \wedge d v \\
& =d f \wedge(P d u)+d f \wedge(Q d v)+f(d P \wedge d u+d Q \wedge d v) \\
& =d f \wedge \omega+f d \omega
\end{aligned}
$$

Traremos, brevemente, o conceito de terna de formas diferenciais. Todas definições e resultados que seguem são inteiramente análogos ao caso das 0-formas,1-formas e 2-formas diferenciais.

Se $F: U \subset \mathbb{R}^{2} \rightarrow \mathbb{R}^{3}$ dada por $(u, v) \mapsto F(u, v)=\left(F^{1}(u, v), F^{2}(u, v), F^{3}(u, v)\right)$ é uma função diferenciável, temos que em cada $q \in U$, sua diferencial é um transformação linear entre $\mathbb{R}^{2}$ e $\mathbb{R}^{3}$ dada por

$$
d F_{q}: \mathbb{R}^{2} \rightarrow \mathbb{R}^{3} ; \quad V \in \mathbb{R}^{2} \mapsto d F_{q}(V)=\left(d F_{q}^{1}(V), d F_{q}^{2}(V), d F_{q}^{3}(V)\right)
$$

Deste modo é natural definirmos $d F$ como uma terna de 1-formas diferenciais $\left(d F^{1}, d F^{2}, d F^{3}\right)$. Isto motiva a seguinte definição.

Definição 2.8. (Terna de 1-formas diferenciáves) Dizemos que $\Omega$ é uma terna (ordenada) de 1-formas diferenciais em $U \subset \mathbb{R}^{2}$, desde que, $\Omega$ seja uma associação que a cada $q \in U$ corresponda a uma transformação linear de $\mathbb{R}^{2}$ em $\mathbb{R}^{3}$ dada por;

$$
\Omega_{q}=\left(\omega_{q}^{1}, \omega_{q}^{2}, \omega_{q}^{3}\right),
$$

em que cada $\omega^{j}$ seja uma 1-forma diferencial em $U$.

Definimos, naturalmente, a soma entre duas ternas, $\Omega, \bar{\Omega}$ e produto por 0 -formas, $h$ por;

$$
(\Omega+h \bar{\Omega})_{q}=\left(\omega_{q}^{1}+h(q) \bar{\omega}_{q}^{1}, \omega_{q}^{2}+h(q) \bar{\omega}_{q}^{2}, \omega_{q}^{3}+h(q) \bar{\omega}_{q}^{3}\right)
$$

Note que, neste contexto, $F=\left(F^{1}, F^{2}, F^{3}\right)$ é uma terna de 0 -formas diferenciais. Além disso, denotando $F \omega=\left(F^{1} w, F^{2} w, F^{3} w\right)$ com $\omega$ uma 1-forma diferencial temos que a seguinte 
expressão;

$$
\begin{aligned}
d F & =\left(d F^{1}, d F^{2}, d F^{3}\right) \\
& =\left(F_{u}^{1} d u+F_{v}^{1} d v, F_{u}^{2} d u+F_{v}^{2} d v, F_{u}^{3} d u+F_{v}^{3} d v\right) \\
& =\left(F_{u}^{1} d u, F_{u}^{2} d u, F_{u}^{3} d u\right)+\left(F_{v}^{1} d v, F_{v}^{2} d v, F_{v}^{3} d v\right) \\
& =\left(F_{u}^{1}, F_{u}^{2}, F_{u}^{3}\right) d u+\left(F_{v}^{1}, F_{v}^{2}, F_{v}^{3}\right) \\
& =F_{u} d u+F_{v} d v
\end{aligned}
$$

Analogamente as definições anteriores, vamos definir uma terna de 2-formas diferenciais para então definir diferencias exteriores de ternas de 1-formas diferenciais.

Definição 2.9. (Terna de 2-formas diferenciáveis) Dizemos que $\Phi$ é uma terna de 2-formas diferenciais em $U \subset \mathbb{R}^{2}$, desde que, $\Phi$ seja uma associação que a cada $q \in U$, a uma aplicação

$$
\Phi_{q}: \mathbb{R}^{2} \times \mathbb{R}^{2} \rightarrow \mathbb{R} ; \quad \Phi_{q}=\left(\phi_{q}^{1}, \phi_{q}^{2}, \phi_{q}^{3}\right),
$$

em que cada $\phi^{j}$ é uma 2-forma diferencial em $U$.

Definiremos a soma de duas ternas de 2 -formas diferenciais, $\Phi, \bar{\phi}$, e a multiplicação por uma 0-forma diferencial, $h$ como

$$
(\Phi+h \bar{\Phi})_{q}=\left(\phi_{q}^{1}+h(q) \bar{\phi}_{q}^{1}, \phi_{q}^{2}+h(q) \bar{\phi}_{q}^{2}, \phi_{q}^{3}+h(q) \bar{\phi}_{q}^{3}\right)
$$

Com o conceito de terna de 1-formas diferenciais, podemos definir a diferencial exterior de uma terna de 1 -formas diferenciais, como a seguir.

Definição 2.10. (Diferencial Exterior de ternas de 1-formas diferenciais) Seja $\Omega=\left(\omega^{1}, \omega^{2}, \omega^{3}\right)$ uma terna de 1-formas diferenciais em um aberto $U \subset \mathbb{R}^{2}$. Definimos a diferencial exterior de $\Omega$ pela terna de 2-formas diferenciais, $d \Omega$, dada por

$$
d \Omega=\left(d \omega^{1}, d \omega^{2}, d \omega^{3}\right)
$$

Novamente, esta definição é extendida para a terna de 0 -formas, de modo que se $F=$ $\left(F^{1}, F^{2}, F^{3}\right)$ é uma função de $U \subset \mathbb{R}^{2}$ em $\mathbb{R}^{3}$, sua diferencial exterior é a terna de 1 -formas diferenciais dada por

$$
d F=\left(d F^{1}, d F^{2}, d F^{3}\right) .
$$

Além disso, ainda vale que $d(d F)=\left(d\left(d F^{1}\right), d\left(d F^{2}\right), d\left(d F^{3}\right)\right) \equiv 0$.

Por último discutiremos o produto exterior entre terna de 1-formas diferenciais e 1-formas diferenciais, 
Definição 2.11. Sejam $\Omega=\left(\omega^{1}, \omega^{2}, \omega^{3}\right)$ uma terna de 1-formas diferenciais definidas em $U$ e $\bar{\omega}$ uma 1-forma diferencial em $U$, definimos o produto exterior entre elas pela terna de 2-formas diferenciais, $\Omega \wedge \bar{\omega}$, por

$$
\Omega \wedge \bar{w}=\left(\omega^{1} \wedge \bar{w}, \omega^{2} \wedge \bar{w}, \omega^{3} \wedge \bar{w}\right)
$$

Analogamente definimos $\bar{w} \wedge \Omega$.

Esta definição nos permite expressar a diferencial de uma terna de 1-formas diferenciais $\Omega=\left(\omega^{1}, \omega^{2}, \omega^{3}\right), \operatorname{com} \omega^{j}=P^{j} d u+Q^{j} d v$, por,

$$
\begin{aligned}
d \Omega & =\left(d \omega^{1}, d \omega^{2}, d \omega^{3}\right) \\
& =\left(d P^{1} \wedge d u+d Q^{1} \wedge d v, \ldots, d P^{3} \wedge d u+d Q^{3} \wedge d v\right) \\
& =\left(d P^{1}, d P^{2}, d P^{3}\right) \wedge d u+\left(d Q^{1}, d Q^{2}, d Q^{3}\right) \wedge d v \\
& =d P \wedge d u+d Q \wedge d v
\end{aligned}
$$

Além disso, pela definição de terna, teremos que as diferenciais e produto exterior de ternas de 1-formas diferenciais, herdarão as propriedades de diferencial e produto exterior de 1-formas diferenciais.

\subsection{Método do Referencial Móvel em $\mathbb{L}^{3}$}

Nesta seção introduziremos o Método do Referencial Móvel. Este método fornece uma descrição alternativa para tratar a Geometria Diferencial e, de fato, alguns autores a utilizam para introduzir a Geometria Diferencial ( [3], [6] e [18]). A vantagem deste método é sua notação, clara e enxuta, que simplifica bastante algumas contas e demonstrações. Mas em contrapartida ela requerer a introdução do conceito de formas diferenciais.

Começaremos introduzindo o Método do Referencial Móvel para superfícies parametrizadas em $\mathbb{E}^{3}$ seguindo a referência [20]. Em seguida, adaptaremos este método para superfícies parametrizadas em $\mathbb{L}^{3}$. Com esta sequência, ficam claras as diferenças e semelhanças deste método nos dois casos $\mathbb{E}^{3}$ e $\mathbb{L}^{3}$.

O Método do Referencial Móvel foi introduzido pelo matemático francês Élie Joseph Cartan e consiste em fixar um referencial ortonormal especial (dito Referencial Móvel) ao longo de cada ponto da uma superfície. A partir deste referencial construir um conjunto de 1-formas diferenciais (dito co-Referencial Móvel) quais traduzirão a geometria da superfície, 
isto é, poderemos expressar a primeira e segunda formas fundamentais em termos destas 1-formas diferenciais, e todos seus derivados, como curvatura principal, curvatura Gaussiana, equações de Gauss e Codazzi e etc. A vantagem imediata deste método consiste na liberdade de escolha do referencial móvel, na qual uma escolha adequada, poderá colocar em evidência o aspecto geométrico que se deseja tratar.

\subsubsection{Referenciais Móveis em $\mathbb{E}^{3}$}

Ao longo desta seção, consideremos $X: U \subset \mathbb{R}^{2} \rightarrow \mathbb{E}^{3}$ uma superfície parametrizada e $U \subset \mathbb{R}^{2}$ um subconjunto aberto, conexo de $\mathbb{R}^{2}$ e $T_{q} X$ o espaço tangente a $X$ em $q \in U$.

Definição 2.12. Um Referencial Móvel (ou Triedro Móvel) adaptado a superfície $\boldsymbol{X}$ é uma terna de funções diferenciáveis $e_{i}: U \subset \mathbb{R}^{2} \rightarrow \mathbb{R}^{3}, i=1,2,3$, tais que, para cada $q \in U$ o conjunto $\left\{e_{1}(q), e_{2}(q), e_{3}(q)\right\}$ é uma base ortonormal de $\mathbb{R}^{3}$ e $e_{1}(q), e_{2}(q)$ pertencem a $T_{q} X$ o espaço tangente a superfície $X$ no ponto $q$.

Observação 2.5. Naturalmente, se $\left\{e_{1}(q), e_{2}(q), e_{3}(q)\right\}$ é um referencial móvel adaptado a $X$, então a condição de ser uma base ortonormal implica em $\left\langle\left\{e_{1}(q), e_{2}(q)\right\}\right\rangle=T_{q} X$ e $\left\langle\left\{e_{3}(q)\right\}\right\rangle=\left(T_{q} X\right)^{\perp}$, em que $\perp$ é tomado em relação ao espaço $\mathbb{E}^{3}$.

Note que dada uma superfície parametrizada $X$ sempre existe um referencial móvel adaptado a $X$. De fato, basta considerar

$$
e_{3}=\frac{X_{u} \times_{\mathbb{E}^{3}} X_{v}}{\left|X_{u} \times_{\mathbb{E}^{3}} X_{v}\right|_{\mathbb{E}^{3}}}, \quad e_{1}=\frac{X_{u}}{\left|X_{u}\right|_{\mathbb{E}^{3}}} \quad \text { e } \quad e_{2}=e_{3} \times_{\mathbb{E}^{3}} e_{1}
$$

Seja $\left\{e_{1}(q), e_{2}(q), e_{3}(q)\right\}$ um referencial móvel adaptado a $X$. Para cada $q \in U$ e para cada $V \in \mathbb{R}^{2}$ temos que $d X_{q}(V) \in T_{q} X$. Como $\left\{e_{1}(q), e_{2}(q)\right\}$ gera $T_{q} X$ podemos expressar

$$
d X_{q}(V)=\left(\omega^{1}\right)_{q}(V) e_{1}(q)+\left(\omega^{2}\right)_{q}(V) e_{2}(q)
$$

em que $\left(\omega^{i}\right)_{q}(V)$ são números reais dados pelas componentes do vetor tangente $d X_{q}(V)$ na base do referencial móvel, isto é,

$$
\left(\omega^{1}\right)_{q}(V)=\left\langle d X_{q}(V), e_{1}(q)\right\rangle_{\mathbb{E}^{3}}, \quad\left(\omega^{2}\right)_{q}(V)=\left\langle d X_{q}(V), e_{2}(q)\right\rangle_{\mathbb{E}^{3}}
$$

Analogamente, como cada função $e_{i}$ é diferenciável em $U$, temos que para cada $q \in U$ a diferencial $d\left(e_{i}\right)_{q}$ é uma transformação que associa a cada $V \in \mathbb{R}^{2}$ a um vetor $d\left(e_{i}\right)_{q}(V) \in \mathbb{R}^{3}$, e uma vez que $\left\{e_{1}(q), e_{2}(q), e_{3}(q)\right\}$ gera $\mathbb{R}^{3}$, temos

$$
d\left(e_{i}\right)_{q}(V)=\left(\omega_{1}^{i}\right)_{q}(V) e_{1}(q)+\left(\omega_{i}^{2}\right)_{q}(V) e_{2}(q)+\left(\omega_{i}^{3}\right)_{q}(V) e_{3}(q), \quad i=1,2,3
$$


em que os escalares são dados por

$$
\left(\omega_{i}^{j}\right) q(V)=\left\langle d\left(e_{i}\right)_{q}(V), e_{j}\right\rangle_{\mathbb{E}^{3}}, \quad i, j \in\{1,2,3\} .
$$

Considerando estas decomposições para cada $q \in U$ e para cada $V \in \mathbb{R}^{2}$, ficam definidas as 1-formas diferenciais em $U$ tais que a cada $q \in U$ é associado um elemento do espaço dual $\left(\mathbb{R}^{2}\right)^{*}$ dado respectivamente por $\left(\omega^{i}\right)_{q}$ e $\left(\omega_{i}^{j}\right)_{q}: \mathbb{R}^{2} \rightarrow \mathbb{R}, i, j \in\{1,2,3\}$.

Por outro lado, $X, e_{1}, e_{2}, e_{3}$ são funções diferenciáveis de $U \subset \mathbb{R}^{2}$ em $\mathbb{R}^{3}$ de modo que suas diferenciais induzem ternas de 1-formas diferenciais. Isto é,

$$
\begin{aligned}
d X & =\left(d X^{1}, d X^{2}, d X^{3}\right) \\
& =\left(X_{u}^{1} d u+X_{v}^{1} d v, X_{u}^{1} 2 d u+X_{v}^{2} d v, X_{u}^{3} d u+X_{v}^{3} d v\right) \\
& =\left(X_{u}^{1}, X_{u}^{2}, X_{u}^{3}\right) d u+\left(X_{v}^{1}, X_{v}^{2}, X_{v}^{3}\right) d v \\
& =X_{u} d u+X_{v} d v
\end{aligned}
$$

Assim a expressão explícita de $\omega^{i}$ é

$$
\omega^{i}=\left\langle X_{u} d u+X_{v} d v, e_{i}\right\rangle_{\mathbb{E}^{3}}=\left\langle X_{u}, e_{i}\right\rangle_{\mathbb{E}^{3}} d u+\left\langle X_{v}, e_{i}\right\rangle_{\mathbb{E}^{3}} d v
$$

Analogamente,

$$
\omega_{i}^{j}=\left\langle\left(e_{i}\right)_{u}, e_{j}\right\rangle_{\mathbb{E}^{3}} d u+\left\langle\left(e_{i}\right)_{v}, e_{j}\right\rangle_{\mathbb{E}^{3}} d v .
$$

Estas 1-formas diferenciais, assim construídas, desempenham um papel fundamental no Método do referencial móvel. Para estas formas temos a seguinte definição.

Definição 2.13. Seja $X$ uma superfície parametrizada em $U \subset \mathbb{R}^{2}$ e $\left\{e_{1}(q), e_{2}(q), e_{3}(q)\right\}$ um referencial móvel adaptado a $X$. Dizemos que $\left\{\omega^{1}, \omega^{2}\right\}$, com $\omega^{i}=\left\langle d X, e_{i}\right\rangle_{\mathbb{E}^{3}}$, é um coreferencial móvel adaptado a $X$ em $U$ e as 1-formas $w_{i}^{j}=\left\langle d e_{i}, e_{j}\right\rangle_{\mathbb{E}^{3}}$ com $i, j \in\{1,2,3\}$ são ditas as formas de conexão.

Proposição 2.4. Seja $\left\{e_{1}, e_{2}, e_{3}\right\}$ um referencial móvel adaptado a superfície parametrizada $X$ em U. Então o co-referencial móvel $\left\{\omega^{1}, \omega^{2}\right\}$ formam uma base para o espaço das 1-formas diferenciais em $U$.

Demonstração: Uma vez que o co-referencial é composto por duas 1-formas diferenciais em $U$, basta mostrar que elas são linearmente independentes segundo a Definição 2.3. Primeiramente os vetores $X_{u}$ e $X_{v}$ são linearmente independentes em $\mathbb{R}^{3}$. Assim se escrevermos $X_{u}=a_{11} e_{1}+a_{21} e_{2}$ e $X_{v}=a_{12} e_{1}+a_{22} e_{2}$ teremos que

$$
\left|\begin{array}{ll}
a_{11} & a_{12} \\
a_{21} & a_{22}
\end{array}\right| \neq 0 .
$$


Deste modo podemos escrever, para $i \in\{1,2\}$,

$$
w^{i}=\left\langle X_{u}, e_{i}\right\rangle_{\mathbb{E}^{3}} d u+\left\langle X_{v}, e_{i}\right\rangle_{\mathbb{E}^{3}} d v=a_{i 1} d u+a_{i 2} d v
$$

Avaliemos então o produto exterior $\omega^{1} \wedge \omega^{2}$;

$$
\begin{aligned}
\omega^{1} \wedge \omega^{2} & =\left(a_{11} d u+a_{12}\right) d u \wedge\left(a_{21} d u+a_{22}\right) d v \\
& =a_{11} a_{22} d u \wedge d v+a_{12} a_{21} d v \wedge d u \\
& =\left(a_{11} a_{22}-a_{12} a_{21}\right) d u \wedge d v \neq 0 .
\end{aligned}
$$

Assim, pela Proposição 2.1 temos que as formas $\omega^{1}$ e $\omega^{2}$ são, de fato, linearmente independentes e o resultado segue.

Proposição 2.5. Sejam $X: U \subset \mathbb{R}^{2} \rightarrow \mathbb{E}^{3}$ uma superfície parametrizada em $\mathbb{E}^{3}$ e $\left\{e_{1}, e_{2}, e_{3}\right\}$ um referencial móvel adaptado a superfície $X$. Então o co-referencial, $\omega^{i}$, e as formas de conexão $\omega_{j}^{i}$ satisfazem

$$
\begin{gathered}
\omega_{j}^{i}=-\omega_{i}^{j} \\
d \omega^{1}=\omega^{2} \wedge \omega_{2}^{1} \\
d \omega^{2}=\omega^{1} \wedge \omega_{1}^{2} \\
\omega^{1} \wedge \omega_{1}^{3}+\omega^{2} \wedge \omega_{2}^{3}=0 \\
d \omega_{1}^{2}=\omega_{1}^{3} \wedge \omega_{3}^{2} \\
d \omega_{1}^{3}=\omega_{1}^{2} \wedge \omega_{2}^{3} \\
d \omega_{2}^{3}=\omega_{2}^{1} \wedge \omega_{1}^{3}
\end{gathered}
$$

Demonstração: Dado um referencial móvel $\left\{e_{1}, e_{2}, e_{3}\right\}$, temos por ortonormalidade que $\left\langle e_{i}, e_{j}\right\rangle_{\mathbb{E}^{3}}=\delta_{i j}$, assim tomando a derivada dos dois lados, temos

$$
\begin{gathered}
\left\langle d e_{i}, e_{j}\right\rangle_{\mathbb{E}^{3}}+\left\langle e_{i}, d e_{j}\right\rangle_{\mathbb{E}^{3}}=0 \\
\omega_{i}^{j}+\omega_{j}^{i}=0,
\end{gathered}
$$

o que mostra a Equação 2.2. Note que esta equação acarreta que $\omega_{i}^{i}=0$ para todo $i=1,2,3$. 
Uma vez que $X$ é uma terna de funções diferenciáveis temos que $d(d X)=0$, assim temos que

$$
\begin{aligned}
0 & =d\left(\omega^{1} e_{1}+\omega^{2} e_{2}\right) \\
& =d e_{1} \wedge \omega^{1}+e_{1} d \omega_{1}+d e_{2} \wedge \omega^{2}+e_{2} d \omega^{2} \\
& =\left(\omega_{1}^{2} e_{2}+\omega_{1}^{3} e_{3}\right) \wedge \omega^{1}+e_{1} d \omega^{1}+\left(\omega_{2}^{1} e_{1}+\omega_{2}^{3} e_{3}\right) \wedge \omega^{2}+e_{2} d \omega^{2} \\
& =\left(\omega_{2}^{1} \wedge \omega^{2}+d \omega^{1}\right) e_{1}+\left(\omega_{1}^{2} \wedge \omega^{1}+d \omega^{2}\right) e_{2}+\left(\omega_{1}^{3} \wedge \omega^{1}+\omega_{2}^{3} \wedge \omega^{2}\right) e_{3} \\
& =\left(-\omega^{2} \wedge \omega_{2}^{1}+d \omega^{1}\right) e_{1}+\left(-\omega^{1} \wedge \omega_{1}^{2}+d \omega^{2}\right) e_{2}-\left(\omega^{1} \wedge \omega_{1}^{3}+\omega^{2} \wedge \omega_{2}^{3}\right) e_{3}
\end{aligned}
$$

Logo, usando a independência linear do referencial móvel em cada $q \in U$, temos que cada componente desta expressão vetorial deve ser nula em cada $q$, e assim temos as equações (2.3)-(2.5).

Obtemos as últimas equações de modo análogo. Usando que $e_{i}$ é uma função diferenciável devemos ter para cada $i \in\{1,2,3\}$, que

$$
\begin{aligned}
0 & =d\left(d e_{i}\right) \\
& =d\left(\sum_{j=1}^{3} \omega_{i}^{j} e_{j}\right) \\
& =\sum_{j=1}^{3}\left[d\left(e_{j}\right) \wedge \omega_{i}^{j}+d \omega_{i}^{j} e_{j}\right] \\
& =\sum_{j=1}^{3}\left[\left(\sum_{k=1}^{3} \omega_{i}^{k} e_{k}\right) \wedge \omega_{i}^{j}+d \omega_{i}^{j} e_{j}\right] \\
& =\sum_{j=1}^{3} \sum_{k=1}^{3}\left[\left(\omega_{i}^{k} \wedge \omega_{i}^{j}\right) e_{k}+d \omega_{i}^{k} e_{k}\right] \\
& =\sum_{k=1}^{3}\left[\sum_{j=1}^{3}\left(\omega_{i}^{k} \wedge \omega_{i}^{j}\right)+d \omega_{i}^{k}\right] e_{k} .
\end{aligned}
$$

Novamente, usando a independência linear da referencial móvel devemos ter que para cada $k \in\{1,2,3\}$

$$
d \omega_{i}^{k}=-\sum_{j=1}^{3}\left(\omega_{i}^{k} \wedge \omega_{i}^{j}\right) \quad i, k \in\{1,2,3\} .
$$

Deste modo, quando $i=1$ e $k=2$, temos

$$
\begin{aligned}
& d \omega_{1}^{2}=-\omega_{1}^{2} \wedge \omega_{1}^{1}-\omega_{1}^{2} \wedge \omega_{1}^{2}-\omega_{1}^{2} \wedge \omega_{1}^{3} \\
& d \omega_{1}^{2}=-\omega_{1}^{2} \wedge 0-0-\omega_{1}^{2} \wedge \omega_{1}^{3} \\
& d \omega_{1}^{2}=\omega_{1}^{2} \wedge \omega_{3}^{1} .
\end{aligned}
$$


De onde temos a equação (2.6).

De maneira análoga, quando $i=1$ e $k=3$ temos a equação (2.7) e por último, quando $i=2$ e $k=3$ temos a equação 2.8 . Os demais casos se reduzem a identidade $0=0$. Com isto demonstramos a Proposição.

O conjunto de Equações (2.2)-(2.8) formam um conjunto de equações necessárias para existência de qualquer co-referencial móvel. Elas representam um papel análogo ao das equações de compatibilidade na teoria local das superfícies.

Definição 2.14. Sejam $X: U \subset \mathbb{R}^{2} \rightarrow \mathbb{E}^{3}$ uma superfície parametrizada em $\mathbb{E}^{3}$ e $\left\{e_{1}, e_{2}, e_{3}\right\}$ um referencial móvel adaptado a superfície $X$. Dizemos que as equações (2.2)-(2.8) são as Equações de Estrutura do referencial móvel, as equações 2.3)-2.5 são ditas Equação de Cartan a equação (2.6) é dita Equação de Gauss e por último (2.7)-(2.8) são ditas Equações Codazzi.

Note que, como o co-referencial $\omega^{1}$ e $\omega^{2}$ forma uma base para o espaço das 1-formas em $U \subset \mathbb{R}^{2}$, podemos escrever as formas de conexão $\omega_{i}^{j}$ nesta base. A equação de estrutura $2.2, \omega_{i}^{j}=-\omega_{j}^{i}$, nos assegura que se conhecermos as seis componentes de $\omega_{1}^{2}, \omega_{1}^{3}$ e $\omega_{2}^{3}$ na base do co-referencial podemos construir todas as demais formas de conexão. Além disso, a equação de estrutura 2.5), $\omega^{1} \wedge \omega_{1}^{3}+\omega^{2} \wedge \omega_{2}^{3}=0$, eliminam implicitamente uma destas seis componentes. Do mesmo modo as equações 2.3 e (2.4) elimina, implicitamente, duas das cinco componentes restantes, a saber, exatamente as duas componentes da 1-forma $\omega_{1}^{2}$. Finalmente as três últimas equações de estrutura (2.6)-(2.8) nos dão relações implícitas entre as três componentes restantes. Implicitamente, isto quer dizer que quaisquer outras expressões, que envolvam demais derivadas exteriores das formas de conexão ou do co-referencial, não fornecerão informações novas e serão identidades já conhecidas ou identidades triviais do tipo $0=0$.

Precisando algumas das afirmações acima, temos as duas seguintes proposições.

Proposição 2.6. Seja $X$ uma superfície parametrizada com um referencial móvel $\left\{e_{1}, e_{2}, e_{3}\right\}$, então as formas de conexões $\omega_{1}^{3}=h_{11} \omega^{1}+h_{12} \omega^{2}$ e $\omega_{2}^{3}=h_{21} \omega^{1}+h_{22} \omega^{2}$ expressas na base do co-referencial móvel pelos coeficientes $h_{i j}$ são tais que $h_{12}=h_{21}$. 
Demonstração: De fato, decorre da equação de estrutura (2.5) que

$$
\begin{aligned}
0 & =\omega^{1} \wedge \omega_{1}^{3}+\omega^{2} \wedge \omega_{2}^{3} \\
& =\omega^{1} \wedge\left(h_{11} \omega^{1}+h_{12} \omega^{2}\right)+\omega^{2} \wedge\left(h_{21} \omega^{1}+h_{22} \omega^{2}\right) \\
& =h_{12} \omega^{1} \wedge \omega^{2}+h_{21} \omega^{2} \wedge \omega^{1} \\
& =\left(h_{12}-h_{21}\right) \omega^{1} \wedge \omega^{2}
\end{aligned}
$$

E assim a tese decorre da independência linear entre $\omega^{1}$ e $\omega^{2}$ usando o Corolário (2.1).

Proposição 2.7. (Lema de Cartan) Seja X uma superfície parametrizada com um referencial móvel $\left\{e_{1}, e_{2}, e_{3}\right\}$. Então a forma de conexão $\omega_{1}^{2}$ é determinada implicitamente, na base do co-referencial móvel, pelas equações de estrutura $d \omega^{1}=\omega^{2} \wedge \omega_{2}^{1}$ e d $\omega^{2}=\omega^{1} \wedge \omega_{1}^{2}$.

Demonstração: De fato, suponhamos que existam duas 1-formas, digamos $\omega_{1}^{2}$ e $\bar{\omega}_{1}^{2}$, satisfazendo as equações de estrutura (2.3) e 2.4. Como o co-referencial móvel é uma base, temos que a 1-forma dada pela diferença $\omega_{1}^{2}-\bar{\omega}_{1}^{2}=A \omega^{1}+B \omega^{2}$ é expressa nesta base para algum par de coeficientes $A$ e $B$.

Primeiramente a equação de estrutura (2.3) nos assegura que

$$
\omega^{2} \wedge \omega_{1}^{2}=\omega^{2} \wedge \bar{\omega}_{1}^{2}
$$

O que equivale a

$$
\begin{aligned}
0 & =\omega^{2} \wedge\left(\omega_{1}^{2}-\bar{w}_{1}^{2}\right) \\
& =\omega^{2} \wedge\left(A \omega^{1}+B \omega^{2}\right) \\
& =-A \omega^{1} \wedge \omega^{2}
\end{aligned}
$$

isto é, $A=0$.

Analogamente a equação de estrutura (2.4) implicará que o coeficiente $B=0$.

De modo que a diferença é nula e concluímos que $\omega_{1}^{2}=\bar{\omega}_{1}^{2}$.

A seguir vamos relacionar o co-referencial móvel e as formas de conexão de uma superfície $X$ com suas primeira e segunda formas fundamentais. Primeiramente apresentemos uma caracterização de superfícies parametrizadas em termos de 1-formas diferenciais. 
Teorema 2.1. Suponha que $X: U \subset \mathbb{R}^{2} \rightarrow \mathbb{R}^{3}$ seja uma aplicação diferenciável em $U$ aberto de $\mathbb{R}^{2}$. Então para que $X$ seja uma superfície parametrizada regular em $\mathbb{E}^{3}$ é necessário e suficiente que existam $f, \bar{f}: U \subset \mathbb{R}^{2} \rightarrow \mathbb{R}^{3}$ diferenciáveis em $U$ e um par de 1-formas diferenciais $\omega, \bar{\omega}$ em $U$ satisfazendo as seguintes condições:

a) $f(q), \bar{f}(q)$ são dois vetores linearmente independentes em $\mathbb{R}^{3}$ para cada $q \in U$.

b)As 1-formas $\omega$ e $\bar{\omega}$ são linearmente independentes.

c) Em cada $q \in U, d X_{q}=\omega_{q} f(q)+\bar{w}_{q} \bar{f}(q)$.

Demonstração: Para a condição necessária, suponha que $X$ é uma superfície parametrizada regular. Tome as funções $f=X_{u}$ e $\bar{f}=X_{v}$ e as 1-formas $\omega=d u$ e $\bar{w}=d v$. Note que $X$ ser regular implica que a condição a) é satisfeita. As 1-formas $d u$ e $d v$ são linearmente independentes, assim temos a condição b). Por último a expressão $d X_{q}=X_{u} d u+X_{v} d v$ garante condição c).

Para a condição suficiente, basta mostrar que, nestas condições, $d X_{q}$ é uma aplicação injetiva para cada $q \in U$. Pelo teorema do núcleo e da imagem isto equivale a garantir que sempre que um vetor $V \in \mathbb{R}^{2}$ for tal que $d X_{q}(V)=0$ devemos ter que $V=0$. Suponha que existam funções diferenciáveis $f$ e $\bar{f}$ e um par de 1-formas $\omega$ e $\bar{\omega}$ tais que as condições a)-c) são satisfeitas. Seja $V \in \mathbb{R}^{2}$ um vetor tal que $d X(V)=0$. Pela condição c), teremos

$$
\omega_{q}(V) f(q)+\bar{\omega}(V) \bar{f}(q)=0 .
$$

A independência linear entre $f(q)$ e $\bar{f}(q)$ dada pela condição a) nos garante que

$$
\omega_{q}(V)=\bar{\omega}(V)=0
$$

Assim para todo vetor $\bar{V}$ temos

$$
(\omega \wedge \bar{w})_{q}(V, \bar{V})=\omega_{q}(V) \bar{\omega}_{q}(\bar{V})-\bar{\omega}_{q}(V) \omega_{q}(\bar{V})=0 .
$$

Suponhamos, por contradição, que $V \neq 0$ de modo que existe $\bar{V}$ tal que $\{V, \bar{V}\}$ é uma base de $\mathbb{R}^{2}$. Dados quaisquer par de vetor de $\mathbb{R}^{2}$ podemos escrevê-los nesta base como $\mu V+\eta \bar{V}$ e $\alpha V+\beta \bar{V}$. Pela bilinearidade da aplicação $(\omega \wedge \bar{w})_{q}$ devemos ter

$$
\begin{aligned}
(\omega \wedge \bar{w})_{q}(\mu V+\eta \bar{V}, \alpha V+\beta \bar{V})= & (\omega \wedge \bar{w})_{q}(V, \bar{V})(\mu \beta-\eta \alpha)+ \\
& +(\omega \wedge \bar{\omega})_{q}(V, V)(\mu \alpha)+ \\
& +(\omega \wedge \bar{\omega})_{q}(\bar{V}, \bar{V})(\eta \beta)=0
\end{aligned}
$$


pois, conforme a Observação (2.4), esta é uma forma alternada e assim os dois últimos termos se anulam. Isto significa que $(\omega \wedge \bar{w})_{q} \equiv 0$ o que é um absurdo perante a hipótese dada pela condição b). Assim, garantimos que $V=0$ e consequente $X$ é uma superfície parametrizada regular em $\mathbb{E}^{3}$.

Observação 2.6. Ainda nas hipóteses do teorema acima, para cada $q \in U$ temos que as funções $f$ e $\bar{f}$ em $q$ geram o espaço tangente a superfície em $q$.

Sabemos que em uma superfície parametrizada $X$, as primeira e segunda formas quadráticas fundamentais são aplicações, em um ponto $q \in U, I_{q}, I I_{q}: T_{q} X \rightarrow \mathbb{R}$ dadas respectivamente por se $V \in \mathbb{R}^{2}$

$$
\begin{gathered}
I_{q}\left(d X_{q}(V)\right)=\left\langle d X_{q}(V), d X_{q}(V)\right\rangle_{\mathbb{E}^{3}}, \\
I I_{q}\left(d X_{q}(V)\right)=-\left\langle d N_{q}(V), d X_{q}(V)\right\rangle_{\mathbb{E}^{3}},
\end{gathered}
$$

em que $d N_{q}$ é a diferencial da aplicação normal $N: U \subset \mathbb{R}^{2} \rightarrow \mathbb{R}^{3}$ em $q$.

Deste modo, se $\left\{e_{1}, e_{2}, e_{3}\right\}$ é um referencial móvel adaptado a superfície $X$ temos que $e_{3}=N$ é normal a superfície. Assim seja $\omega^{i}$ o co-referencial móvel e $\omega_{i}^{j}$ as formas de conexão. Deverá valer que $d X=\omega^{1} e_{1}+\omega^{2} e_{2}$ e $d N=d e_{3}$, de modo que

$$
\begin{aligned}
I_{q}(d X(V)) & =\left\langle d X_{q}(V), d X_{q}(V)\right\rangle_{\mathbb{E}^{3}} \\
& =\left(\omega_{q}^{1}(V)\right)^{2}+\left(\omega_{q}^{2}(V)\right)^{2}
\end{aligned}
$$

Esta igualdade para todo $V \in \mathbb{R}^{2}$ e para cada $q \in U$ induz a identidade

$$
I=\omega^{1} \otimes \omega^{1}+\omega^{2} \otimes \omega^{2} .
$$

Analogamente;

$$
\begin{aligned}
I I & =-\langle d X, d N\rangle_{\mathbb{E}^{3}} \\
& =-\left\langle\omega^{1} e_{1}+\omega^{2} e_{2}, \omega_{3}^{1} e_{1}+\omega_{3}^{2} e_{2}\right\rangle_{\mathbb{E}^{3}} \\
& =-\omega^{1} \otimes \omega_{3}^{1}-\omega^{2} \otimes \omega_{3}^{2} \\
& =\omega^{1} \otimes \omega_{1}^{3}+\omega^{2} \otimes \omega_{2}^{3} .
\end{aligned}
$$

Deste modo, uma vez que a primeira e segunda forma fundamental é expressa em termos do co-referencial móvel e das formas de conexão podemos expressar os entes geométricos de $X$ em termos destas formas diferenciais, como por exemplo a curvatura Gaussiana de $X$. 
Teorema 2.2. Sejam $X$ uma superfície parametrizada regular em $\mathbb{E}^{3}$ com curvatura Gaussiana $K,\left\{e_{1}, e_{2}, e_{3}\right\}$ um referencial móvel adaptado a $X$ com $\left\{\omega^{1}, \omega^{2}\right\}$ co-referencial e $\left\{\omega_{i}^{j}\right\}$ formas de conexão. Então a seguinte relação vale,

$$
d \omega_{1}^{2}=-K \omega^{1} \wedge \omega^{2}
$$

A demonstração segue analogamente do caso de superfícies no espaço de Minkowski, qual será feita em detalhes na próxima seção, no Teorema 2.3 .

\subsubsection{Referenciais Móveis em $\mathbb{L}^{3}$}

Nesta seção trataremos dos referenciais móveis em superfícies parametrizadas em $\mathbb{L}^{3}$. A motivação para construção do método do referencial móvel em $\mathbb{L}^{3}$ é a mesma do caso de superfícies em $\mathbb{E}^{3}$ de modo que a exposição desta seção será análoga a exposição da seção anterior. Existem diferenças na construção do método do referencial móvel em cada aspecto em que se faz referência a conceitos métricos, como por exemplo na definição de um referencial móvel onde o conceito de "ortonormalidade"é diferente entre os espaços $\mathbb{E}^{3}$ e $\mathbb{L}^{3}$. Em contrapartida, todos os conceitos que não são métricos serão mantidos, como por exemplo as definição de 1-formas diferenciais em um aberto $U \subset \mathbb{R}^{2}$ e suas propriedades.

Ao longo desta seção consideremos $\mathbb{X}: U \subset \mathbb{R}^{2} \rightarrow \mathbb{L}^{3}, U$ um conjunto aberto e conexo de $\mathbb{R}^{2}$, uma superfície parametrizada em $\mathbb{L}^{3}$.

Definição 2.15. Dizemos que uma terna de funções diferenciáveis $e_{1}, e_{2}, e_{3}: U \subset \mathbb{R}^{2} \rightarrow \mathbb{L}^{3}$ formam um Referencial Móvel (ou um Triedro Móvel), desde que, em cada $q \in U$ o conjunto $\left\{e_{1}(q), e_{2}(q), e_{3}(q)\right\}$ formam uma base ortonormal de $\mathbb{L}^{3}$ e $e_{1}(q), e_{2}(q) \in T_{q} \mathbb{X}$.

Notação 2.1. A cada referencial móvel adaptado a uma superfície $\mathbb{X}$, digamos $\left\{e_{1}, e_{2}, e_{3}\right\}$, denotaremos $\epsilon_{i}=\left\langle e_{i}, e_{i}\right\rangle$. Além disso, nos referiremos a função $\epsilon_{i}$, que assume valores em $\{-1,0,+1\}$, como sendo o sinal do vetor $e_{i}$.

Observação 2.7. Note que a cada superfície $\mathbb{X}$ temos que $\left\{e_{1}(q), e_{2}(q)\right\}$ forma uma base para o espaço tangente em $q \in U$. Além disso, se $\mathbb{X}$ é não-degenerada (vide Definição 1.19 ), $e_{3}(q)$ é normal a superfície, em $q \in U$, e seu sinal $\epsilon_{3}(q)$ é 1 se a superfície for tipo-tempo em $q$ ou igual a -1 se a superfície for tipo-espaço em $q$.

Seja $\left\{e_{1}, e_{2}, e_{3}\right\}$ um referencial móvel adaptado a superfície $\mathbb{X}$. De maneira análoga ao caso Euclidiano, ficam definidas 1-formas diferenciais em $U$ da seguinte forma: a cada $q \in U$ 
é associado os seguintes elementos do espaço dual de $\mathbb{R}^{2}$

$$
\begin{gathered}
\left(\omega^{i}\right)_{q}: \mathbb{R}^{2} \rightarrow \mathbb{R} ; \quad V \in \mathbb{R}^{2} \mapsto\left(\omega^{i}\right)_{q}(V)=\left\langle d \mathbb{X}_{q}(V), e_{i}\right\rangle, \\
\left(\omega_{j}^{l}\right)_{q}: \mathbb{R}^{2} \rightarrow \mathbb{R} ; \quad V \in \mathbb{R}^{2} \mapsto\left(\omega_{j}^{l}\right)_{q}(V)=\left\langle d\left(e_{j}\right)_{q}(V), e_{l},\right\rangle
\end{gathered}
$$

em que $i \in\{1,2\}$ e $j, l \in\{1,2,3\}$. Deste modo, podemos denotar as ternas de 1 -formas diferenciais $d \mathbb{X}$ e $d e_{j}$ respectivamente por;

$$
\begin{array}{r}
d \mathbb{X}=\epsilon_{1} \omega^{1} e_{1}+\epsilon_{2} \omega^{2} e_{2} \\
d e_{j}=\epsilon_{1} \omega_{j}^{1} e_{1}+\epsilon_{2} \omega_{j}^{2} e_{2}+\epsilon_{3} \omega_{j}^{3} e_{3}
\end{array}
$$

Estas 1-formas diferenciais cumprirão o mesmo papel que no caso Euclidiano, de modo que temos a seguinte definição;

Definição 2.16. Seja $\mathbb{X}: U \subset \mathbb{R}^{2} \rightarrow \mathbb{L}^{3}$ uma superfície parametrizada em $\mathbb{L}^{3}$ e considere $\left\{e_{1}, e_{2}, e_{3}\right\}$ um referencial móvel adaptado a superfície $\mathbb{X}$. Dizemos que o conjunto de 1 -formas diferenciais $\omega^{1}, \omega^{2}$, definidas em 2.14):

$$
\omega^{i}=\left\langle d \mathbb{X}, e_{i}\right\rangle \quad i \in\{1,2\},
$$

compõem um co-referencial móvel adaptado a superfície $\mathbb{X}$ em $\mathbb{L}^{3}$ e as formas diferenciais $\omega_{j}^{i}$, dadas em 2.15);

$$
\omega_{i}^{j}=\left\langle d e_{i}, e_{j}\right\rangle, \quad i \in\{1,2\} \quad j \in\{1,2,3\},
$$

são ditas formas de conexão associadas ao co-referencial móvel.

Observação 2.8. Alguns autores definem o co-rerencial móvel e as formas de conexão, digamos $\bar{\omega}^{i}$ e $\bar{\omega}_{i}^{j}$, pela expressão

$$
\begin{array}{r}
d \mathbb{X}=\bar{\omega}^{1} e_{1}+\bar{\omega}^{2} e_{2}, \\
d e_{j}=\bar{\omega}_{j}^{1} e_{1}+\bar{\omega}_{j}^{2} e_{2}+\bar{\omega}_{j}^{3} e_{3}
\end{array}
$$

De modo que $\bar{\omega}^{i}=\epsilon_{i}\left\langle d \mathbb{X}, e_{i}\right\rangle=\epsilon_{i} \omega^{i}$ e $\bar{\omega}_{i}^{j}=\epsilon_{j}\left\langle d e_{i}, e_{j}\right\rangle=\epsilon_{j} \omega_{i}^{j}$. Não existe nenhuma diferença significativa entre estas duas definições.

Lema 2.2. Sejam $\mathbb{X}: U \subset \mathbb{R}^{2} \rightarrow \mathbb{L}^{3}$, uma superfície parametrizada regular em $\mathbb{L}^{3}$, e $\left\{e_{1}, e_{2}, e_{3}\right\}$ um referencial móvel adaptado a superfície $\mathbb{X}$. Então o co-referencial móvel $\omega^{1} e$ $\omega^{2}$ formam uma base para o espaço das 1-formas diferenciais em $U \subset \mathbb{R}^{2}$. 
Demonstração: De fato, conforme a expressão 2.14 temos que $\left(\omega^{i}\right)_{q}(\bullet)=\left\langle d \mathbb{X}_{q}(\bullet), e^{i}\right\rangle$ é um elemento do espaço dual de $\mathbb{R}^{2}$ para cada $q \in U$. Como $\mathbb{X}$ é diferenciável podemos escrever a terna de 1 -formas diferenciais $d \mathbb{X}=\mathbb{X}_{u} d u+\mathbb{X}_{v} d v$. Consequentemente

$$
\omega^{i}=\left\langle\mathbb{X}_{u}, e_{i}\right\rangle d u+\left\langle\mathbb{X}_{v}, e_{i}\right\rangle d v
$$

Como $\mathbb{X}$ e $e_{i}$ são funções diferenciáveis em $U$, segue que $\omega^{i}$ é de fato uma 1-forma diferencial em $U$ conforme a Definição 2.1). Nos resta mostrar que $\omega^{1}, \omega^{2}$ são linearmente independentes. Primeiramente, podemos escrever

$$
\begin{aligned}
\mathbb{X}_{u} & =\epsilon_{1}\left\langle\mathbb{X}_{u}, e_{1}\right\rangle e_{1}+\epsilon_{2}\left\langle\mathbb{X}_{u}, e_{2}\right\rangle e_{2} \\
\mathbb{X}_{v} & =\epsilon_{1}\left\langle\mathbb{X}_{v}, e_{1}\right\rangle e_{1}+\epsilon_{2}\left\langle\mathbb{X}_{v}, e_{2}\right\rangle e_{2}
\end{aligned}
$$

Como $\mathbb{X}$ é regular, temos que a diferencial $d \mathbb{X}$ é injetiva o que implica em $\mathbb{X}_{u}$ e $\mathbb{X}_{v}$ serem linearmente independentes. Assim

$$
0 \neq \operatorname{det}\left(\begin{array}{cc}
\epsilon_{1}\left\langle\mathbb{X}_{u}, e_{1}\right\rangle & \epsilon_{1}\left\langle\mathbb{X}_{v}, e_{1}\right\rangle \\
\epsilon_{2}\left\langle\mathbb{X}_{u}, e_{2}\right\rangle & \epsilon_{2}\left\langle\mathbb{X}_{v}, e_{2}\right\rangle
\end{array}\right)=\epsilon_{1} \epsilon_{2} \operatorname{det}\left(\begin{array}{cc}
\left\langle\mathbb{X}_{u}, e_{1}\right\rangle & \left\langle\mathbb{X}_{v}, e_{1}\right\rangle \\
\left\langle\mathbb{X}_{u}, e_{2}\right\rangle & \left\langle\mathbb{X}_{v}, e_{2}\right\rangle
\end{array}\right)
$$

Por outro lado, pela expressão $\omega^{i}=\left\langle\mathbb{X}_{u}, e_{i}\right\rangle d u+\left\langle\mathbb{X}_{v}, e_{i}\right\rangle d v$, temos

$$
\omega^{1} \wedge \omega^{2}=\operatorname{det}\left(\begin{array}{cc}
\left\langle\mathbb{X}_{u}, e_{1}\right\rangle & \left\langle\mathbb{X}_{v}, e_{1}\right\rangle \\
\left\langle\mathbb{X}_{u}, e_{2}\right\rangle & \left\langle\mathbb{X}_{v}, e_{2}\right\rangle
\end{array}\right) d u \wedge d v
$$

assim, $\omega^{1} \wedge \omega^{2} \neq 0$. E portanto pela caracterização dada no Corolário 2.1] segue que $\omega^{1}, \omega^{2}$ são linearmente independentes.

Com este Lema, dado um referencial móvel de uma superfície, podemos escrever as formas de conexão na base do co-referencial móvel. Analogamente ao caso Euclidiano temos a seguinte Proposição.

Proposição 2.8. Sejam $\mathbb{X}: U \subset \mathbb{R}^{2} \rightarrow \mathbb{L}^{3}$, uma superfície parametrizada regular em $\mathbb{L}^{3} e$ $\left\{e_{1}, e_{2}, e_{3}\right\}$ um referencial móvel adaptado a superfície $\mathbb{X}$. Então o co-referencial móvel $\omega^{i} e$ 
as formas de conexão $\omega_{i}^{j}$ satisfazem as seguintes relações;

$$
\begin{gathered}
\omega_{i}^{j}=-\omega_{j}^{i} \\
d \omega^{1}=\epsilon_{2} \omega^{2} \wedge \omega_{2}^{1} \\
d \omega^{2}=\epsilon_{1} \omega^{1} \wedge \omega_{1}^{2} \\
\epsilon_{1} \omega^{1} \wedge \omega_{1}^{3}+\epsilon_{2} \omega^{2} \wedge \omega_{2}^{3}=0 \\
d \omega_{1}^{2}=\epsilon_{3} \omega_{1}^{3} \wedge \omega_{3}^{2} \\
d \omega_{1}^{3}=\epsilon_{2} \omega_{1}^{2} \wedge \omega_{2}^{3} \\
d \omega_{2}^{3}=\epsilon_{1} \omega_{2}^{1} \wedge \omega_{1}^{3} .
\end{gathered}
$$

Demonstração: A demonstração é inteiramente análoga ao caso Euclidiano dada na Proposição 2.5). A ortogonalidade do referencial móvel implicará na equação 2.16). A diferenciabilidade da aplicação $\mathbb{X}$ implicará nas equações 2.17$)-(2.19)$ e por último a diferenciabilidade do referencial móvel implicará nas equações 2.20)-2.22).

De fato, dado um referencial móvel $\left\{e_{1}, e_{2}, e_{3}\right\}$, temos por ortonormalidade que $\left\langle e_{i}, e_{j}\right\rangle=\epsilon_{i} \delta_{i j}$. Assim tomando a derivada dos dois lados, temos

$$
\begin{gathered}
\left\langle d e_{i}, e_{j}\right\rangle+\left\langle e_{i}, d e_{j}\right\rangle=0 \\
\omega_{i}^{j}+\omega_{j}^{i}=0,
\end{gathered}
$$

e consequentemente temos 2.16 .

Usando que $\mathbb{X}$ é uma terna de funções diferenciáveis temos que $d(d \mathbb{X})=0$, e pela relação $d \mathbb{X}=\epsilon_{1} \omega^{1} e_{1}+\epsilon_{2} \omega^{2} e_{2}$, e usando que $\omega_{i}^{i}=0$, temos

$$
\begin{aligned}
0= & d(d \mathbb{X}) \\
0= & d\left(\epsilon_{1} \omega^{1} e_{1}+\epsilon_{2} \omega^{2} e_{2}\right) \\
= & \epsilon_{1} d e_{1} \wedge \omega^{1}+\epsilon_{1} e_{1} d \omega_{1}+\epsilon_{2} d e_{2} \wedge \omega^{2}+\epsilon_{2} e_{2} d \omega^{2} \\
= & \epsilon_{1}\left(\epsilon_{2} \omega_{1}^{2} e_{2}+\epsilon_{3} \omega_{1}^{3} e_{3}\right) \wedge \omega^{1}+\epsilon_{1} e_{1} d \omega^{1}+ \\
& +\epsilon_{2}\left(\epsilon_{1} \omega_{2}^{1} e_{1}+\epsilon_{3} \omega_{2}^{3} e_{3}\right) \wedge \omega^{2}+\epsilon_{2} e_{2} d \omega^{2} \\
= & \left(\epsilon_{2} \epsilon_{1} \omega_{2}^{1} \wedge \omega^{2}+\epsilon_{1} d \omega^{1}\right) e_{1}+\left(\epsilon_{1} \epsilon_{2} \omega_{1}^{2} \wedge \omega^{1}+\epsilon_{2} d \omega^{2}\right) e_{2}+ \\
& +\left(\epsilon_{1} \epsilon_{2} \omega_{1}^{3} \wedge \omega^{1}+\epsilon_{2} \epsilon_{3} \omega_{2}^{3} \wedge \omega^{2}\right) e_{3} .
\end{aligned}
$$

Logo, usando a independência linear do referencial móvel em cada $q \in U$ temos que cada componente desta expressão vetorial deve ser nula em cada $q$ e assim temos as equações 
(2.17)-2.19)

Por último, usando que $e_{i}$ é uma função diferenciável e usando a relação $d e_{i}=\sum_{j=1}^{3} \epsilon_{j} \omega_{i}^{j} e_{j}$, segue que,

$$
\begin{aligned}
0 & =d\left(d e_{i}\right) \\
& =d\left(\sum_{j=1}^{3} \epsilon_{j} \omega_{i}^{j} e_{j}\right) \\
& =\sum_{j=1}^{3}\left[\epsilon_{j} d\left(e_{j}\right) \wedge \omega_{i}^{j}+\epsilon_{j} d \omega_{i}^{j} e_{j}\right] \\
& =\sum_{j=1}^{3}\left[\epsilon_{j}\left(\sum_{k=1}^{3} \epsilon_{k} \omega_{j}^{k} e_{k}\right) \wedge \omega_{i}^{j}+\epsilon_{j} d \omega_{i}^{j} e_{j}\right] \\
& =\sum_{j=1}^{3} \sum_{k=1}^{3}\left[\epsilon_{k} \epsilon_{j}\left(\omega_{j}^{k} \wedge \omega_{i}^{j}\right) e_{k}+\epsilon_{k} d \omega_{i}^{k} e_{k}\right] \\
& =\sum_{k=1}^{3} \epsilon_{k}\left[\sum_{j=1}^{3} \epsilon_{j}\left(\omega_{j}^{k} \wedge \omega_{i}^{j}\right)+d \omega_{i}^{k}\right] e_{k} .
\end{aligned}
$$

Usando a independência linear da referencial móvel devemos ter que para cada $k \in\{1,2,3\}$

$$
d \omega_{i}^{k}=-\sum_{j=1}^{3} \epsilon_{j}\left(\omega_{j}^{k} \wedge \omega_{i}^{j}\right) \quad i, k \in\{1,2,3\} .
$$

Deste modo, quando $i=1$ e $k=2$ :

$$
\begin{aligned}
d \omega_{1}^{2} & =-\epsilon_{1} \omega_{1}^{2} \wedge \omega_{1}^{1}-\epsilon_{2} \omega_{1}^{2} \wedge \omega_{1}^{2}-\epsilon_{3} \omega_{3}^{2} \wedge \omega_{1}^{3} \\
& =-\epsilon_{1} \omega_{1}^{2} \wedge 0-\epsilon_{2} 0-\epsilon_{3} \omega_{3}^{2} \wedge \omega_{1}^{3} \\
& =\epsilon_{3} \omega_{1}^{3} \wedge \omega_{3}^{2}
\end{aligned}
$$

donde temos a equação 2.20 .

De maneira análoga, quando $i=1$ e $k=3$ temos a equação 2.21 e por último, quando $i=2$ e $k=3$ temos a equação 2.22 . Os demais casos se reduzem a identidade $0=0$. Com isto demonstramos a Proposição.

Esta Proposição é o principal resultado deste capítulo, pois as equações 2.16-2.22, sempre serão válidas em qualquer referencial móvel. Elas nos darão informações geométricas importantes sobre a superfície pois, conforme veremos, elas satisfazem um papel análogo 
ao das equações de compatibilidade na teoria local de superfícies. Analogamente ao caso Euclidiano, temos a seguinte definição.

Definição 2.17. Sejam $X: U \subset \mathbb{R}^{2} \rightarrow \mathbb{L}^{3}$ uma superfície parametrizada em $\mathbb{L}^{3}$ e $\left\{e_{1}, e_{2}, 2_{3}\right\}$ um referencial móvel adaptado a superfície $X$. Dizemos que as equações 2.16)-(2.22) são as Equações de Estrutura do referencial móvel, as equações 2.17)-2.19) são ditas as Equações de Cartan a equação (2.20) é dita Equação de Gauss e por último (2.21)(2.22) são ditas Equações Codazzi.

De maneira análoga ao que foi no caso Euclidiano é possível enunciar e demonstrar os análogos do Lema de Cartan dados na Proposição (2.6) e Proposição 2.7) para o caso de $\mathbb{X}$ superfície em $\mathbb{L}^{3}$.

Proposição 2.9. (Lema de Cartan)Sejam $X: U \subset \mathbb{R}^{2} \rightarrow \mathbb{L}^{3}$ uma superfície parametrizada $e m \mathbb{L}^{3}$ e $\left\{e_{1}, e_{2}, e_{3}\right\}$ um referencial móvel adaptado a superfície $X$. Então a forma de conexão $w_{1}^{2}$ é unicamente determinada pelo co-referencial móvel $\omega^{1}, \omega^{2}$.

Proposição 2.10. Sejam $X: U \subset \mathbb{R}^{2} \rightarrow \mathbb{L}^{3}$ uma superfície parametrizada em $\mathbb{L}^{3} e$ $\left\{e_{1}, e_{2}, 2_{3}\right\}$ um referencial móvel adaptado a superfície $X$. Então as primeira e segunda formas fundamentais da superfície são dadas em termos do co-referencial $\omega^{i}$ e das formas de conexão $\omega_{i}^{j}$.

Demonstração: Sabemos que para uma superfície parametrizada $\mathbb{X}$, as primeira e segunda formas quadráticas fundamentais são aplicações, em um ponto $q \in U, I_{q}, I I_{q}: T_{q} X \rightarrow \mathbb{R}$ dadas respectivamente por

$$
\begin{gathered}
I_{q}\left(d X_{q}(V)\right)=\left\langle d X_{q}(V), d X_{q}(V)\right\rangle, \\
I I_{q}\left(d X_{q}(V)\right)=-\left\langle d N_{q}(V), d X_{q}(V)\right\rangle,
\end{gathered}
$$

com $V \in \mathbb{R}^{2}$ e $d N_{q}$ a diferencial da aplicação normal em $q$.

Deste modo, se $\left\{e_{1}, e_{2}, e_{3}\right\}$ é um referencial móvel adaptado a superfície $\mathbb{X}$ temos que $e_{3}=N$ é normal a superfície. Assim, seja $\omega^{i}$ o co-referencial móvel, e $\omega_{i}^{j}$ as formas de conexão. Deverá valer que $d X=\epsilon_{1} \omega^{1} e_{1}+\epsilon_{2} \omega^{2} e_{2}$ e $d N=d e_{3}$, de modo que

$$
\begin{aligned}
I_{q}(d X(V)) & =\left\langle d X_{q}(V), d X_{q}(V)\right\rangle \\
& =\left(\omega_{q}^{1}(V)\right)^{2}+\left(\omega_{q}^{2}(V)\right)^{2}
\end{aligned}
$$


Esta igualdade para todo $V \in \mathbb{R}^{2}$ e para cada $q \in U$ induz a identidade

$$
I=\omega^{1} \otimes \omega^{1}+\omega^{2} \otimes \omega^{2}
$$

Analogamente

$$
\begin{aligned}
I I & =-\langle d X, d N\rangle \\
& =-\left\langle\epsilon_{1} \omega^{1} e_{1}+\epsilon_{2} \omega^{2} e_{2}, \epsilon_{1} \omega_{3}^{1} e_{1}+\epsilon_{2} \omega_{3}^{2} e_{2}\right\rangle \\
& =-\left(\epsilon_{1}\right)^{2} \omega^{1} \otimes \omega_{3}^{1}-\left(\epsilon_{2}\right)^{2} \omega^{2} \otimes \omega_{3}^{2} \\
& =\omega^{1} \otimes \omega_{1}^{3}+\omega^{2} \otimes \omega_{2}^{3} .
\end{aligned}
$$

De modo que expressamos as primeira e segunda formas fundamentais em termos do coreferencial móvel e das formas de conexão.

O seguinte teorema nos dá uma forma de relacionar o co-referencial móvel com a curvatura Gaussiana da superfície, e também justifica a nomenclatura de Equação de Gauss para a Equação 2.20.

Teorema 2.3. Sejam $\mathbb{X}: U \subset \mathbb{R}^{2} \rightarrow \mathbb{L}^{3}$ uma superfície parametrizada regular não-degenderada, $\left\{e_{1}, e_{2}, e_{3}\right\}$ um referencial móvel adaptado a superfície $\mathbb{X}$ e $K$ a curvatura Gaussiana da superfície $\mathbb{X}$. Então o co-referencial móvel é tal que vale a expressão

$$
d \omega_{1}^{2}=-K \omega^{1} \wedge \omega^{2}
$$

Demonstração: Para mostrar que vale a relação $\omega_{1}^{2}=-K \omega^{1} \wedge \omega^{2}$ entre duas 2-formas diferenciais podemos usar a bilinearidade de uma 2-forma e mostrar que as imagens, por alguma base de $\mathbb{R}^{2}$, coincidem.

Como $\mathbb{X}$ é uma superfície parametrizada regular e $\left\{e_{1}, e_{2}\right\}$ formam uma base de $T_{q} \mathbb{X}$, em cada $q \in U$, garantimos que $f^{1}=d \mathbb{X}_{q}^{-1}\left(e_{1}\right) \in \mathbb{R}^{2}$ e $f^{2}=d \mathbb{X}_{q}^{-1}\left(e_{2}\right) \in \mathbb{R}^{2}$ forma uma base para $\mathbb{R}^{2}$.

Por definição, uma 2-forma diferencial é uma forma alternada, de modo que se verifica automaticamente a identidade;

$$
\left(d \omega_{1}^{2}\right)_{q}\left(f^{i}, f^{i}\right)=(-1) K(q)\left(\omega^{1} \wedge \omega^{2}\right)_{q}\left(f^{i}, f^{i}\right), \quad i=1,2
$$

Nos resta mostrar o caso não trivial. Por definição $K(q)=\epsilon_{3} \operatorname{det}\left(S_{q}\right)$ em que $S_{q}: T_{q} \mathbb{X} \rightarrow$ $T_{q} \mathbb{X}$ é o Operador de Weingarten da superfície $\mathbb{X}$ em $q \in U$. Ainda vale que $S_{q}(x)=$ 
$-d N_{q}\left(d \mathbb{X}^{-1}(x)\right)=-d\left(e_{3}\right)_{q}\left(d \mathbb{X}^{-1}(x)\right)$, com $x \in T_{q} \mathbb{X}$. Expressando na base $\left\{e_{1}(q), e_{2}(q)\right\}$ de $T_{q} \mathbb{X}$ temos que a matriz deste operador é dada por;

$$
\begin{aligned}
S_{q} & \doteq\left(\begin{array}{cc}
\epsilon_{1}\left\langle S_{q}\left(e_{1}\right), e_{1}\right\rangle & \epsilon_{1}\left\langle S_{q}\left(e_{2}\right), e_{1}\right\rangle \\
\epsilon_{2}\left\langle S_{q}\left(e_{1}\right), e_{2}\right\rangle & \epsilon_{2}\left\langle S_{q}\left(e_{2}\right), e_{2}\right\rangle
\end{array}\right) \\
& =\left(\begin{array}{cc}
\epsilon_{1}\left\langle-d\left(e_{3}\right)_{q}\left(f^{1}\right), e_{1}\right\rangle & \epsilon_{1}\left\langle-d\left(e_{3}\right)_{q}\left(f^{2}\right), e_{1}\right\rangle \\
\epsilon_{2}\left\langle-d\left(e_{3}\right)_{q}\left(f^{1}\right), e_{2}\right\rangle & \epsilon_{2}\left\langle-d\left(e_{3}\right)_{q}\left(f^{2}\right), e_{2}\right\rangle
\end{array}\right) \\
& =\left(\begin{array}{cc}
-\epsilon_{1}\left(\omega_{3}^{1}\right)_{q}\left(f^{1}\right) & -\epsilon_{1}\left(\omega_{3}^{1}\right)_{q}\left(f^{2}\right) \\
-\epsilon_{2}\left(\omega_{3}^{2}\right)_{q}\left(f^{1}\right) & -\epsilon_{2}\left(\omega_{3}^{2}\right)_{q}\left(f^{2}\right)
\end{array}\right)
\end{aligned}
$$

De modo que,

$$
\begin{aligned}
K(q) & =\epsilon_{3} \operatorname{det}\left(S_{q}\right) \\
& =\epsilon_{3}\left(\epsilon_{1} \epsilon_{2}\left(\omega_{3}^{1}\right)_{q}\left(f^{1}\right)\left(\omega_{3}^{2}\right)_{q}\left(f^{2}\right)-\epsilon_{1} \epsilon_{2}\left(\omega_{3}^{1}\right)_{q}\left(f^{2}\right)\left(\omega_{3}^{2}\right)_{q}\left(f^{1}\right)\right) \\
& =\epsilon_{3}\left(\epsilon_{2} \epsilon_{1}\left(\omega_{3}^{1} \otimes \omega_{3}^{2}\right)_{q}\left(f^{1}, f^{2}\right)-\epsilon_{2} \epsilon_{1}\left(\omega_{3}^{2} \otimes \omega_{3}^{1}\right)_{q}\left(f^{1}, f^{2}\right)\right) \\
& =\epsilon_{3} \epsilon_{2} \epsilon_{1}\left(\omega_{3}^{1} \wedge \omega_{3}^{2}\right)_{q}\left(f^{1}, f^{2}\right) .
\end{aligned}
$$

Usando que $\epsilon_{1} \epsilon_{2} \epsilon_{3}=-1$ (pois, sendo a superfície $\mathbb{X}$ não-degenerada, somente um dos sinais $\epsilon_{i}$ é idêntico a -1 e os outros dois restantes são idênticos a +1 ) e usando que $\omega_{3}^{1}=-\omega_{1}^{3}$,

$$
K(q)=\left(\omega_{1}^{3} \wedge \omega_{3}^{2}\right)_{q}\left(f^{1}, f^{2}\right) .
$$

Usando agora a Equação de estrutura 2.20 $\left(d \omega_{1}^{2}=\epsilon_{3} \omega_{1}^{3} \wedge \omega_{3}^{2}\right)$, teremos

$$
d \omega_{1}^{2}\left(f^{1}, f^{2}\right)=\epsilon_{3} K(q) .
$$

Por outro lado, note que,

$$
\omega^{i}\left(f^{j}\right)=\left\langle d \mathbb{X}\left(f^{j}\right), e_{i}\right\rangle=\left\langle e_{j}, e_{i}\right\rangle=\epsilon_{i} \delta_{i j},
$$

deste modo,

$$
\begin{aligned}
\left(\omega^{1} \wedge \omega^{2}\right)_{q}\left(f^{1}, f^{2}\right) & =\left(\omega^{1} \otimes \omega^{2}\right)_{q}\left(f^{1}, f^{2}\right)-\left(\omega^{2} \otimes \omega^{1}\right)_{q}\left(f^{1}, f^{2}\right) \\
& =\left(\omega_{1}\right)_{q}\left(f^{1}\right)\left(\omega_{2}\right)_{q}\left(f^{2}\right)-\left(\omega_{2}\right)_{q}\left(f^{1}\right)\left(\omega_{1}\right)_{q}\left(f^{2}\right) \\
& =\epsilon_{1} \epsilon_{2}=-\epsilon_{3} .
\end{aligned}
$$

Em que na última igualdade usamos novamente a relação $\epsilon_{1} \epsilon_{2} \epsilon_{3}=-1$. E assim, substituindo $\epsilon_{3}$ desta equação na relação 2.25 , obtemos

$$
d \omega_{1}^{2}\left(f^{1}, f^{2}\right)=-K(q)\left(\omega^{1} \wedge \omega^{2}\right)_{q}\left(f^{1}, f^{2}\right) .
$$


Assim garantimos que

$$
d \omega_{1}^{2}=-K\left(\omega^{1} \wedge \omega^{2}\right)
$$

E o teorema está demonstrado.

Este teorema permite escrever a Equação de Gauss de uma superfície em termos das 1formas do co-referencial móvel. Além disso, este teorema juntamente com o Lema de Cartan (Proposição 2.9) permite demonstrar o análogo, para superfícies no espaço de Minkowski, de um dos teoremas mais célebres da Geometria Diferencial.

Corolário 2.2. (Theorema Egregium de Gauss) Seja $\mathbb{X}$ uma superfície parametrizada regular não-degenerada em $\mathbb{L}^{3}$. Então a curvatura Gaussiana de $K$ de $\mathbb{X}$ é definida intrinsecamente, isto é, depende somente da primeira forma fundamental.

Demonstração: Vimos que a não-degenerescência e regularidade de $\mathbb{X}$ implicam na existência de referenciais móveis. E dado qualquer referencial móvel sempre fica bem definido o coreferencial móvel. Pela Proposição 2.10 vimos que sempre é possível expressar a primeira forma fundamental de $\mathbb{X}$ em termos deste co-referencial via equação 2.23 por

$$
I=\omega^{1} \otimes \omega^{1}+\omega^{2} \otimes \omega^{2} .
$$

Sendo $\mathbb{X}$ uma superfície não-degenerada, temos a Equação de Gauss dada pelo Teorema 2.3

$$
d \omega_{1}^{2}=-K \omega^{1} \wedge \omega^{2}
$$

Pelo Lema de Cartan a 1-forma $\omega_{1}^{2}$ é univocamente determinada pelo co-referencial móvel, assim a curvatura depende somente do co-referencial móvel que por sua vez está relacionado com a primeira forma fundamental. Deste modo garantimos que $K$ é um objeto intrínseco da superfície.

\subsubsection{Referenciais Nulos em $\mathbb{L}^{3}$}

Recordamos que na definição de um referencial móvel pedimos que $\left\{e_{1}, e_{2}, e_{3}\right\}$ formasse uma base ortonormal de $\mathbb{L}^{3}$, isto é, $\left\{e_{i}, e_{j}\right\}=\epsilon \delta_{i j}$. Assim construímos o co-referencial móvel usando esta propriedade. 
Nosso objetivo nesta seção é generalizar a noção de referencial móvel e co-referencial móvel para os casos em que o triedro móvel admita vetores tipo-luz. Veremos que tal generalização será útil para tratarmos superfícies tipo-tempo não Weingarten-diagonalizáveis. Denominaremos tais referenciais por Referenciais nulos.

Primeiramente seja $P$ um plano qualquer tipo-tempo. Pela Proposição (1.5), existem $a, b \in P$ vetores tipo-luz linearmente independentes e pela Proposição (1.4) podemos supor que $\langle a, b\rangle=\mu>0$. Existe também $u$ um vetor tipo-espaço normal ao plano $P$. Assim vamos tratar de referenciais do tipo $\{a, b, u\}$.

Definição 2.18. Seja $\mathbb{X}$ uma superfície parametrizada regular tipo-tempo no espaço de Minkowski. Diremos que as aplicações $e_{1}, e_{2}, e_{3}: U \subset \mathbb{R}^{2} \rightarrow \mathbb{L}^{3}$ formam um Referencial Móvel Nulo, ou simplesmente Referencial Nulo, adaptado a superfície $\mathbb{X}$ desde que, sejam aplicações diferenciáveis e em cada ponto q formem uma base de $\mathbb{L}^{3}$ tal que $e_{1}, e_{2}$ são vetores tipo-luz, linearmente independentes com $\left\langle e_{1}, e_{2}\right\rangle=\mu>0$ e e é um vetor tipo-espaço normal.

Definiremos o co-referencial móvel nulo e as formas de conexão, de modo que,

$$
\begin{array}{r}
d \mathbb{X}=\omega^{1} e_{1}+\omega^{2} e_{2} \\
d e_{j}=\omega_{j}^{1} e_{1}+\omega_{j}^{2} e_{2}+\omega_{j}^{3} e_{3} .
\end{array}
$$

Para isto basta tomar;

$$
\begin{aligned}
\omega^{1} & :=\frac{1}{\mu}\left\langle d \mathbb{X}, e_{2}\right\rangle \\
\omega^{2} & :=\frac{1}{\mu}\left\langle d \mathbb{X}, e_{1}\right\rangle \\
\omega_{j}^{1} & :=\frac{1}{\mu}\left\langle d e_{j}, e_{2}\right\rangle \\
\omega_{j}^{2} & :=\frac{1}{\mu}\left\langle d e_{j}, e_{1}\right\rangle \\
\omega_{j}^{3} & :=\left\langle d e_{j}, e_{3}\right\rangle
\end{aligned}
$$

em que $\mu=\mu(q)$ é o parâmetro definido por $\left\langle e_{1}, e_{2}\right\rangle=\mu>0$. De fato para mostrar que estas 1 -formas assim definidas satisfazem 2.26) e 2.27). Basta mostrar que em cada $q$, os vetores $d \mathbb{X}-\omega^{1} e_{1}-\omega^{2} e_{2}$ e $d e_{j}-\omega_{j}^{1} e_{1}-\omega_{j}^{2} e_{2}-\omega_{j}^{3} e_{3}$ são ortogonais a $e_{1}, e_{2}$ e $e_{3}$ e usar que a métrica é não degenerada. 
As equações de estrutura serão construídas de modo análogo:

$$
\begin{array}{r}
\omega_{1}^{2}=\omega_{2}^{1}=\omega_{3}^{3}=0, \quad \omega_{1}^{3}+\mu \omega_{3}^{2}=0, \quad \omega_{2}^{3}+\mu \omega_{3}^{1}=0 \\
d \omega^{1}=\omega^{1} \wedge \omega_{1}^{1} \\
d \omega^{2}=\omega^{2} \wedge \omega_{2}^{2} \\
\omega^{1} \wedge \omega_{1}^{3}+\omega^{2} \wedge \omega_{2}^{3}=0 \\
d \omega_{1}^{1}=\omega_{1}^{3} \wedge \omega_{3}^{1} \\
d \omega_{2}^{2}=\omega_{2}^{3} \wedge \omega_{3}^{2} \quad\left(=-\mu K \omega^{1} \wedge \omega^{2}\right) \\
d \omega_{3}^{1}=\omega_{3}^{1} \wedge \omega_{1}^{1} \\
d \omega_{3}^{2}=\omega_{3}^{2} \wedge \omega_{2}^{2}
\end{array}
$$

em que $K$ é a curvatura Gaussiana da superfície $\mathbb{X}$ e $\mu>0$ é o parâmetro tal que $\left\langle e_{1}, e_{2}\right\rangle=\mu$.

De fato, primeiramente como $\left\langle e_{i}, e_{i}\right\rangle \in\{0,1\}$, temos

$$
\left\langle d e_{i}, e_{i}\right\rangle=0
$$

Tomando $i=2,1,3$ temos a primeira, segunda e terceira equação de 2.34 respectivamente. Partido da relação $\left\langle e_{i}, e_{3}\right\rangle=0$ para $i=1,2$ temos as duas últimas equações de 2.34). Observamos que como definimos $\mu=\mu(p)$ a equação $\left\langle e_{1}, e_{2}\right\rangle=\mu$ em geral não necessariamente implica em uma relação do tipo 2.34.

Usando a diferenciabilidade de $\mathbb{X}$ e as equações que acabamos de demonstrar, temos

$$
\begin{aligned}
0 & =d(d \mathbb{X})=d\left(\omega^{1} e_{1}+\omega^{2} e_{2}\right) \\
& =d \omega^{1} e_{1}+d\left(e_{1}\right) \wedge \omega^{1}+d \omega^{2} e_{2}+d\left(e_{2}\right) \wedge \omega^{2} \\
& =d \omega^{1} e_{1}+\left(\omega_{1}^{1} e_{1}+\omega_{1}^{3} e_{3}\right) \wedge \omega^{1}+d \omega^{2} e_{2}+\left(\omega_{2}^{2} e_{2}+\omega_{2}^{3} e_{3}\right) \wedge \omega^{2} \\
& =\left(d \omega^{1}+\omega_{1}^{1} \wedge \omega^{1}\right) e_{1}+\left(d \omega^{2}+\omega_{2}^{2} \wedge \omega^{2}\right) e_{2}+\left(\omega_{1}^{3} \wedge \omega^{1}+\omega_{2}^{3} \wedge \omega^{2}\right) e_{3},
\end{aligned}
$$

de onde decorre 2.35)-2.37). 
Da diferenciabilidade de $e_{j}$, decorre que

$$
\begin{aligned}
0 & =d\left(d e_{j}\right)=d\left(\sum_{i=1}^{3} \omega_{j}^{i} e_{i}\right) \\
& =\sum_{i=1}^{3} d \omega_{j}^{i} e_{i}+\sum_{i=1}^{3} d e_{i} \wedge \omega_{j}^{i} \\
& =\sum_{i=1}^{3} d \omega_{j}^{i} e_{i}+\sum_{i=1}^{3} \sum_{k=1}^{3}\left(\omega_{i}^{k} e_{k}\right) \wedge \omega_{j}^{i} \\
& =\sum_{k=1}^{3} d \omega_{j}^{k} e_{k}+\sum_{k=1}^{3} \sum_{i=1}^{3} \omega_{i}^{k} \wedge \omega_{j}^{i} e_{k} \\
& =\sum_{k=1}^{3}\left(d \omega_{j}^{k}+\sum_{i=1}^{3} \omega_{i}^{k} \wedge \omega_{j}^{i}\right) e_{k},
\end{aligned}
$$

consequentemente para todo $k=1,2,3$

$$
d \omega_{j}^{k}=\sum_{i=1}^{3} \omega_{j}^{i} \wedge \omega_{i}^{k} .
$$

Assim com $j=1$ e $k=1$, segue que

$$
\begin{aligned}
d \omega_{1}^{1} & =\omega_{1}^{1} \wedge \omega_{1}^{1}+\omega_{1}^{2} \wedge \omega_{2}^{1} \wedge+\omega_{1}^{3} \wedge \omega_{3}^{1} \\
& =\omega_{1}^{3} \wedge \omega_{3}^{1},
\end{aligned}
$$

de onde decorre 2.38). Tomando agora, $j=2$ e $k=2$,

$$
\begin{aligned}
d \omega_{2}^{2} & =\omega_{2}^{1} \wedge \omega_{1}^{2}+\omega_{2}^{2} \wedge \omega_{2}^{2} \wedge+\omega_{2}^{3} \wedge \omega_{3}^{2} \\
& =\omega_{2}^{3} \wedge \omega_{3}^{2}
\end{aligned}
$$

e assim temos (2.39). Com $j=3$ e $k=1$,

$$
\begin{aligned}
d \omega_{3}^{1} & =\omega_{3}^{1} \wedge \omega_{1}^{1}+\omega_{3}^{2} \wedge \omega_{2}^{1} \wedge+\omega_{3}^{3} \wedge \omega_{3}^{1} \\
& =\omega_{3}^{1} \wedge \omega_{1}^{1}
\end{aligned}
$$

donde vale 2.40). Por último (2.41) é obtida considerando $j=3$ e $k=2$. De fato,

$$
\begin{aligned}
d \omega_{3}^{2} & =\omega_{3}^{1} \wedge \omega_{1}^{2}+\omega_{3}^{2} \wedge \omega_{2}^{2}+\omega_{3}^{3} \wedge \omega_{3}^{2} \\
& =\omega_{3}^{2} \wedge \omega_{2}^{2} .
\end{aligned}
$$

Por último, a relação adicional $d \omega_{2}^{2}=-\mu K \omega^{1} \wedge \omega^{2}$ na Equação de Estrutura 2.39 é obtida pela seguinte proposição. Esta equação é dita Equação de Gauss. 
Proposição 2.11. Seja $\mathbb{X}: U \subset \mathbb{R}^{2} \rightarrow \mathbb{L}^{3}$ uma superfície parametrizada regular em $\mathbb{L}^{3}$ com curvatura Gaussiana K. Suponha que $\mathbb{X}$ seja tipo-tempo e tenha um Referencial Nulo adaptado $\left\{e_{i}\right\}$ com parâmetro $\mu=\left\langle e_{1}, e_{2}\right\rangle>0,\left\{\omega^{i}\right\}$ co-referencial e $\left\{\omega_{i}^{j}\right\}$ formas de conexão. Então em cada $q \in U$, temos

$$
d \omega_{2}^{2}=-\mu K \omega^{1} \wedge \omega^{2}
$$

Demonstração: Por definição a curvatura Gaussiana de uma superfície tipo-tempo é $K(q)=$ $\operatorname{det} S_{q}, \operatorname{com} S_{q}$ o operador de Weingarten.

Seja $\left\{f^{1}, f^{2}\right\} \subset \mathbb{R}^{2}$ a base de $\mathbb{R}^{2}$ dada por $f^{i}=d \mathbb{X}_{q}^{-1}\left(e_{i}\right)$, onde $\left\{e_{1}, e_{2}\right\}$ é a base de $T_{q} \mathbb{X}$. Como $S_{q}=-d N_{q}=-d\left(e_{3}\right)_{q}=-\omega_{3}^{1} e_{1}-\omega_{3}^{2} e_{2}$ decorre que a matriz representativa de $S_{q}$ nesta base é dada por,

$$
S_{q} \doteq\left(\begin{array}{cc}
-\omega_{3}^{1}\left(f^{1}\right) & -\omega_{3}^{1}\left(f^{2}\right) \\
-\omega_{3}^{2}\left(f^{1}\right) & -\omega_{3}^{2}\left(f^{2}\right)
\end{array}\right) .
$$

Daí $K=\omega_{3}^{1}\left(f^{1}\right) \omega_{3}^{2}\left(f^{2}\right)-\omega_{3}^{1}\left(f^{2}\right) \omega_{3}^{2}\left(f^{1}\right)$. Usando as equações de estrutura, segue que

$$
\begin{aligned}
K & =\omega_{3}^{1} \wedge \omega_{3}^{2}\left(f^{1}, f^{2}\right) \\
& =\left(-\mu^{-1} \omega_{2}^{3}\right) \wedge \omega_{3}^{2}\left(f^{1}, f^{2}\right) \\
& =-\mu^{-1} d \omega_{2}^{2}\left(f^{1}, f^{2}\right)
\end{aligned}
$$

Agora usando a relação $\omega^{1} \wedge \omega^{2}\left(f^{1}, f^{2}\right)=1$, obtemos

$$
d \omega_{2}^{2}\left(f^{1}, f^{2}\right)=-\mu K=-\mu K \omega^{1} \wedge \omega^{2}\left(f^{1}, f^{2}\right)
$$

Paralelamente para $j=1,2$, obtemos

$$
\begin{aligned}
d \omega_{2}^{2}\left(f^{j}, f^{j}\right) & =\omega_{2}^{3} \wedge \omega_{3}^{2}\left(f^{j}, f^{j}\right) \\
& =\omega_{2}^{3}\left(f^{j}\right) \omega_{3}^{2}\left(f^{j}\right)-\omega_{3}^{2}\left(f^{j}\right) \omega_{2}^{3}\left(f^{j}\right)=0 .
\end{aligned}
$$

Além disso

$$
\omega^{1} \wedge \omega^{2}\left(f^{j}, f^{j}\right)=\omega^{1}\left(f^{j}\right) \omega^{2}\left(f^{j}\right)-\omega^{2}\left(f^{j}\right) \omega^{1}\left(f^{j}\right)=0 .
$$

Portanto, temos que a igualdade, entre 2-formas, se verifica;

$$
d \omega_{2}^{2}=-\mu K \omega^{1} \wedge \omega^{2}
$$

Logo a Proposição está demonstrada. 
Encerraremos essaa seção exibindo a primeira e segunda formas fundamentais da superfície tipo-tempo $\mathbb{X}$ em termos do co-referencial móvel associado ao referencial nulo. Primeiramente,

$$
\begin{aligned}
I_{q} & =\langle d \mathbb{X}, d \mathbb{X}\rangle \\
& =\left\langle\omega^{1} e_{1}+\omega^{2} e_{2}, \omega^{1} e_{1}+\omega^{2} e_{2}\right\rangle \\
& =\omega^{1} \otimes \omega^{2}\left\langle e_{1}, e_{2}\right\rangle+\omega^{2} \otimes \omega^{1}\left\langle e_{2}, e_{1}\right\rangle \\
& =\mu\left(\omega^{1} \otimes \omega^{2}+\omega^{2} \otimes \omega^{1}\right) .
\end{aligned}
$$

E também,

$$
\begin{aligned}
I I_{q} & =\left\langle d \mathbb{X}, S_{q}\right\rangle=\left\langle d \mathbb{X},-d N_{q}\right\rangle \\
& =\left\langle\omega^{1} e_{1}+\omega^{2} e_{2},-\omega_{3}^{1} e_{1}-\omega_{3}^{2} e_{2}\right\rangle \\
& =\omega^{1} \otimes \omega_{3}^{2}\left\langle e_{1},-e_{2}\right\rangle+\omega^{2} \otimes \omega_{3}^{1}\left\langle e_{2},-e_{1}\right\rangle \\
& =-\mu\left(\omega^{1} \otimes \omega_{3}^{2}+\omega^{2} \otimes \omega_{3}^{1}\right) .
\end{aligned}
$$




\section{Capítulo 3}

\section{Superfícies não-degeneradas de}

\section{curvatura constante não nula em $\mathbb{L}^{3}$ e suas EDP's.}

Neste capítulo daremos algumas aplicações do Método do Referencial Móvel. Apresentaremos uma classificação das superfíces não-degeneradas no espaço de Minkowski cuja curvatura Gaussiana é constante. Veremos que a tais superfícies é possível associar uma EDP específica, dita EDP Natural, de tal forma que existirá uma relação biunívoca entre uma solução desta equação e existência de superfície. Mais precisamente, a cada superfície nãodegenerada com curvatura Gaussiana constante será possível associar uma solução de uma EDP e a cada solução desta EDP existirá uma única (a menos de movimento rígido) superfície não-degenerada de curvatura Gaussiana constante.

Os resultados a seguir, assim como outros resultados obtidos pelo método do Referencial Móvel, admitem uma demonstração alternativa sem o uso do método do referencial móvel. Entretanto tais demonstrações são notavelmente mais extensas e mais trabalhosas. Isto de certa forma justifica o emprego do método do referencial móvel.

Notamos que existe um resultado clássico, já conhecido por Bäcklund em 1875 [1], que relaciona as superfícies de curvatura Gaussiana negativa no espaço Euclidiano com sua EDP natural, a saber a equação de sine-Gordon, na qual motivou por muitos anos o estudo das superfícies de curvatura constante negativa. Entretanto, não era conhecido se superfícies de curvatura constante no espaço de Minkowski possuíam tal propriedade. 
Na literatura um dos trabalhos pioneiros que abordaram o tema de superfícies no espaço de Minkowski e suas EDP's naturais, veio com a tese de doutorado da Louise Vincentia McNertney, defendida em 1980 e intitulada " One-parameter families of surfaces with constant curvature in Lorentz 3-space" [15]. Nesta tese, dentre outros resultados, é demonstrado como relacionar as superfícies tipo-tempo de curvatura constante negativa nos casos em que o polinômio característico admite duas raízes ou uma raiz real com soluções das EDP's de sinhGordon negativa $\alpha_{u u}-\alpha_{v v}=-\sinh \alpha$ e a equação de Liouville $\alpha_{u v}=\frac{1}{2} e^{\alpha}$, repectivamente.

Posteriormente, em 1981, S.S. Chern [4], no artigo "Geometrical interpretation of the sinh-Gordon equation", estabelece o método de relacionar as superfícies tipo-espaço com curvatura constante negativa e as superfícies tipo-tempo com curvatura constante positiva imersas em um espaço-forma pseudo-Riemanniano de curvatura constante positiva com suas EDP's naturais. Três anos depois, em 1984, Hu Hesheng [10] no artigo "The Construction of Hyperbolic Surfaces in 3-Dimensional Minkowski Space and Sinh-Gordon Equation" trás uma adaptação do método de Chern para superfícies no espaço de Minkowski ao invés do espaço-forma.

Com os métodos estabelecidos pela L. V. McNertney e por S.S. Chern, nos quais eram essenciais usar parametrizações por linhas de curvatura, foi possível relacionar superfícies não-degeneradas de curvatura constante não nulas que admitiam reparametrizações por linhas de curvatura, com suas EDP's naturais. Entretanto, não se sabia se as superfícies que não adimitem curvaturas principais podiam ou não ser relacionadas com alguma EDP natural.

Cerca duas décadas depois, em 2002, C.H. Gu, H.S. Hu e J. Inoguchi [12 no artigo " On time-like surfaces of positive constant Gaussian curvature and imaginary principal curvatures"trataram este último caso, cuja abordagem é feita com uma adaptação do método da L. V. McNertney para o caso de superfícies tipo-tempo com uma raíz real. Foi estabelecido um Referencial de vetores tipo-luz, para relacionar tais superfícies com soluções da equação de cosh-Gordon $\alpha_{u u}-\alpha_{v v}=\cosh \alpha$.

Neste capítulo colecionaremos os resultados apresentados na literatura, de modo que teremos uma "classificação" de todas as superfícies não-degeneradas no espaço de Minkowski, com curvatura Gaussiana constante não nula em termos de suas EDP's naturais.

Começaremos tratando, na próxima seção, o caso de superfícies com curvatura constante 
no espaço Euclidiano. Esta abordagem de certo modo servirá de "modelo" para obtermos os resultados análogos paras superfícies no espaço de Minkowski. Encerraremos este capítulo resumindo os principais resultados estabelecidos.

\subsection{Superfícies de curvatura Gaussiana constante não nula em $\mathbb{E}^{3}$}

Já é um resultado clássico na literatura que a equação de sine-Gordon está relacionada com as superfícies de curvatura Gaussiana constante negativa. Daremos uma apresentação moderna, usando o método do referencial móvel, do significado geométrico da equação de sine-Gordon.

Nesta seção daremos atenção especial para as superfícies no espaço Euclidiano com curvatura Gaussiana constante negativa, pois, estaremos interessados em construir a Transformação de Bäcklund que acontece somente entrem tais superfícies. No final desta seção apresentaremos brevemente como obter a EDP naturalmente associada as superfícies de curvatura constante positiva.

\subsubsection{Curvatura constante negativa em $\mathbb{E}^{3}$}

Seja $X: U \subset \mathbb{R}^{2} \rightarrow \mathbb{E}^{3}$ uma superfície parametrizada regular com curvatura Gaussiana negativa $K=-1<0$.

Lembramos que um ponto de uma superfície onde a curvatura Gaussiana é negativa, é um ponto não umbílico, isto é, as curvaturas principais $k_{1}$ e $k_{2}$ neste ponto são distintas. Além disso é conhecido, conforme [5] Corolário 4 página 220, que podemos obter uma vizinhança em torno deste ponto tal que as curvas coordenadas sejam linhas de curvatura. Embora todos os pontos da superfície $X$ sejam não umbílicos, não podemos garantir que a reparametrização por linhas de curvatura da vizinhança de um ponto $p_{0}$ seja também uma reparametrização por linhas de curvatura de todos os demais pontos da superfície. Deste modo podemos supor, restringindo o domínio se necessário, que a superfície é tal que as curvas coordenadas são linhas de curvatura.

Deste modo suponha que $X$ esteja parametrizada por $(\bar{u}, \bar{v})$, em que $\left(\bar{u}_{0}, \bar{v}\right)$ e $\left(\bar{u}, \bar{v}_{0}\right)$ são linhas de curvatura. Consideremos um Referencial Móvel $\left\{e_{1}, e_{2}, e_{3}\right\}$ no qual $e_{1}$ e $e_{2}$ são vetores unitários nas direções principais. 
Calculando o co-referencial móvel e usando que $e_{1}$ é perpendicular a $X_{v}$ temos

$$
\omega^{1}=\left\langle d X, e_{1}\right\rangle_{\mathbb{E}^{3}}=\left\langle X_{\bar{u}} d \bar{u}+X_{\bar{v}} d \bar{v}, e_{1}\right\rangle_{\mathbb{E}^{3}}=\left\langle X_{\bar{u}}, e_{1}\right\rangle_{\mathbb{E}^{3}} d \bar{u}
$$

Analogamente,

$$
\omega^{2}=\left\langle X_{\bar{v}}, e_{2}\right\rangle_{\mathbb{E}^{3}} d \bar{v}
$$

Consequentemente $d X=X_{\bar{u}} d \bar{u}+X_{\bar{v}} d \bar{v}$ é expressa, em termos do co-referencial $\omega^{1}, \omega^{2}$, por

$$
d X=\omega^{1} e_{1} d \bar{u}+\omega^{2} e_{2} d \bar{v}
$$

Em geral $d X$ assume a seguinte expressão

$$
d X=\bar{A} e_{1} d \bar{u}+\bar{B} e_{2} d \bar{v}
$$

em que $\bar{A}$ e $\bar{B}$ são funções reais definidas em $U$.

Com isto temos que a primeira forma fundamental, conforme a Equação (2.11), é escrita como

$$
\begin{aligned}
I & =\omega^{1} \otimes \omega^{1}+\omega^{2} \otimes \omega^{2} \\
& =\bar{A}^{2} d \bar{u} \otimes d \bar{u}+\bar{B}^{2} d \bar{v} \otimes d \bar{v} .
\end{aligned}
$$

A segunda forma fundamental, será expressa como

$$
\begin{aligned}
I I & =\langle d X,-d N\rangle_{\mathbb{E}^{3}} \\
& =\left\langle X_{\bar{u}} d \bar{u}+X_{d v} d \bar{v},-N_{\bar{u}} d \bar{u}-N_{\bar{v}} d \bar{v}\right\rangle_{\mathbb{E}^{3}} \\
& =\left\langle X_{\bar{u}} d \bar{u}+X_{d v} d \bar{v},-d N\left(X_{\bar{u}}\right) d \bar{u}-d N\left(X_{\bar{v}}\right) d \bar{v}\right\rangle_{\mathbb{E}^{3}} \\
& =\left\langle X_{\bar{u}} d \bar{u}+X_{\bar{v}} d \bar{v},-d N\left(\bar{A} e_{1}\right) d \bar{u}-d N\left(\bar{B} e_{2}\right) d \bar{v}\right\rangle_{\mathbb{E}^{3}} \\
& =\left\langle X_{\bar{u}} d \bar{u}+X_{\bar{v}} d \bar{v}, \bar{A} k_{1} e_{1} d \bar{u}+\bar{B} k_{2} e_{2} d \bar{v}\right\rangle_{\mathbb{E}^{3}} \\
& =k_{1} \bar{A}\left\langle X_{\bar{u}}, e_{1}\right\rangle_{\mathbb{E}^{3}} d \bar{u} \otimes d \bar{u}+k_{2} \bar{B}\left\langle X_{\bar{v}}, e_{2}\right\rangle_{\mathbb{E}^{3}} d \bar{v} \otimes d \bar{v} \\
& =k_{1} \bar{A}^{2} d \bar{u} \otimes d \bar{u}+k_{2} \bar{B}^{2} d \bar{v} \otimes d \bar{v} .
\end{aligned}
$$

Por outro lado, comparando com a Equação 2.12 temos que

$$
\begin{aligned}
I I & =\omega^{1} \otimes \omega_{1}^{3}+\omega^{2} \otimes \omega_{2}^{3} \\
& =\bar{A} d \bar{u} \otimes \omega_{1}^{3}+\bar{B} d \bar{v} \otimes \omega_{2}^{3} .
\end{aligned}
$$

Assim garantimos que

$$
\begin{gathered}
\omega_{1}^{3}=k_{1} \bar{A} d \bar{u}, \\
\omega_{2}^{3}=k_{2} \bar{B} d \bar{v} .
\end{gathered}
$$


Também, pela Proposição 2.7 podemos determinar a forma de conexão $\omega_{1}^{2}$ usando as equações de estrutura (2.3) e 2.4). Para tanto basta notar que a ansatz

$$
\omega_{1}^{2}=-\frac{\bar{A}_{\bar{v}}}{\bar{B}} d \bar{u}+\frac{\bar{B}_{\bar{u}}}{\bar{A}} d \bar{v}
$$

satisfaz as equações $d \omega^{1}=\omega^{2} \wedge \omega_{2}^{1}$ e $d \omega^{2}=\omega^{1} \wedge \omega_{1}^{2}$. Ou alternativamente escrevendo $\omega_{1}^{2}=P d \bar{u}+Q d \bar{v}$, verifica-se a seguinte cadeia de equivalências

$$
\begin{aligned}
d \omega^{1} & =\omega^{2} \wedge \omega_{2}^{1} \\
d(\bar{A} d \bar{u}) & =(\bar{B} d \bar{v}) \wedge(-P d \bar{u}-Q d \bar{v}) \\
\bar{A}_{\bar{v}} d \bar{v} \wedge d \bar{u} & =-\bar{B} P d \bar{v} \wedge d \bar{u} \\
-\bar{A}_{\bar{v}} d \bar{u} \wedge d \bar{v} & =\bar{B} P d \bar{u} \wedge d \bar{v}
\end{aligned}
$$

donde, $P=-\frac{\bar{A}_{\bar{v}}}{\bar{B}}$. E analogamente usando $d \omega^{2}=\omega^{1} \wedge \omega_{1}^{2}$, obtemos $Q=\frac{\bar{B}_{\bar{u}}}{A}$.

Agora vamos usar as equações de estrutura para obter condições sob as funções $\bar{A}$ e $\bar{B}$. Pela Equação de Codazzi (2.7) segue que

$$
\begin{aligned}
d \omega_{1}^{3} & =\omega_{1}^{2} \wedge \omega_{2}^{3} \\
d\left(k_{1} \bar{A} d \bar{u}\right) & =\left(-\frac{\bar{A}_{\bar{v}}}{\bar{B}} d \bar{u}+\frac{\bar{B}_{\bar{u}}}{\bar{A}} d \bar{v}\right) \wedge\left(k_{2} \bar{B} d \bar{v}\right) \\
\left(k_{1} \bar{A}\right)_{\bar{v}} d \bar{v} \wedge d \bar{u} & =-k_{2} \bar{B} \frac{\bar{A}_{\bar{v}}}{\bar{B}} d \bar{u} \wedge d \bar{v} \\
\left(k_{1} \bar{A}\right)_{\bar{v}} d \bar{u} \wedge d \bar{v} & =k_{2} \bar{B} \bar{A}_{\bar{v}} d \bar{u} \wedge d \bar{v} .
\end{aligned}
$$

Que é equivalente a

$$
\left(k_{1}\right)_{\bar{v}} \bar{A}+\left(k_{1}-k_{2}\right) \bar{A}_{\bar{v}}=0 .
$$

Analogamente a Equação de Codazzi $(2.8) d \omega_{2}^{3}=\omega_{2}^{1} \wedge \omega_{1}^{3}$ é satisfeita se, e somente se,

$$
\left(k_{2}\right)_{\bar{u}} \bar{B}+\left(k_{1}+k_{2}\right) \bar{B}_{\bar{u}}=0 .
$$

Como estamos supondo que a curvatura Gaussiana é uma constante negativa, pelas relações $K=k_{1} k_{2}$ e $k_{1}>k_{2}$, temos que $k_{1}>0$. Logo existe um único $\alpha \in(0, \pi)$ tal que,

$$
k_{1}=\tan (\alpha / 2), \quad \alpha \in(0, \pi)
$$

e consequentemente

$$
k_{2}=-\cot (\alpha / 2), \quad \alpha \in(0, \pi)
$$

Notando que,

$$
\left(k_{1}-k_{2}\right)=\frac{1}{\operatorname{sen}(\alpha / 2) \cos (\alpha / 2)} \quad \text { e } \quad\left(k_{1}\right)_{\bar{v}}=\sec ^{2}(\alpha / 2) \alpha_{\bar{v}} / 2,
$$


temos

$$
\frac{\left(k_{1}\right)_{\bar{v}}}{k_{1}-k_{2}}=\frac{\sin (\alpha / 2)}{\cos (\alpha / 2)} \alpha_{\bar{v}} / 2=-\frac{\partial}{\partial \bar{v}} \ln \cos (\alpha / 2)
$$

Deste modo a Equação (3.7) é equivalente a,

$$
\frac{\partial}{\partial \bar{v}} \ln \bar{A}-\frac{\partial}{\partial \bar{v}} \ln \cos (\alpha / 2)=0 .
$$

Consequentemente $\ln \left(\frac{\bar{A}}{\cos (\alpha / 2)}\right)=a(\bar{u})$ é uma função somente da coordenada $\bar{u}$. Deste modo, garantimos que

$$
\bar{A}=e^{a(\bar{u})} \cos (\alpha / 2) .
$$

Analogamente, da Equação 3.8 existe uma uma função $b(\bar{v})$ tal que

$$
\bar{B}=e^{b(\bar{u})} \sin (\alpha / 2)
$$

Deste modo, fica definido uma reparametrização de $X$ via a mudança de coordenadas $h$, dada por

$$
(\bar{u}, \bar{v}) \rightarrow(u, v) ; \quad(u, v)=h(\bar{u}, \bar{v})=\left(\int^{\bar{u}} e^{a(\xi)} d \xi, \int^{\bar{v}} e^{b(\xi)} d \xi\right)
$$

em que $\int^{x} f(\xi) d \xi$ denota a primitiva da função $f$ no ponto $x$. Observe que $h$ é de fato uma mudança de coordenadas, pois, as funções $a(\bar{u})$ e $b(\bar{v})$ dependem diferencialmente de $\bar{u}$ e $\bar{v}$ e o jacobiano

$$
\left|\begin{array}{cc}
h_{\bar{u}}^{1} & h_{\bar{v}}^{1} \\
h_{\bar{u}}^{2} & h_{\bar{v}}^{2}
\end{array}\right|(\bar{u}, \bar{v})=\left|\begin{array}{cc}
e^{a(\bar{u})} & 0 \\
0 & e^{b(\bar{v})}
\end{array}\right|=e^{a(\bar{u})} e^{b(\bar{v})}>0 .
$$

Deste modo a base $\{d u, d v\}$ das novas coordenadas $(u, v)$, são dadas por

$$
\begin{aligned}
d u & =h_{\bar{u}}^{1} d \bar{u}+h_{\bar{v}}^{1} d \bar{v}=e^{a(\bar{u})} d \bar{u}, \\
d v & =h_{\bar{u}}^{2} d \bar{u}+h_{\bar{v}}^{2} d \bar{v}=e^{b(\bar{v})} d \bar{v} .
\end{aligned}
$$

Retomando as equações (3.1) e 3.2 temos respectivamente,

$$
\begin{gathered}
\omega^{1}=\bar{A} d \bar{u}=\cos (\alpha / 2) d u, \\
\omega^{2}=\bar{B} d \bar{v}=\sin (\alpha / 2) d v .
\end{gathered}
$$

Repetindo as mesmas passagens anteriores considerando agora $\cos (\alpha / 2)$ no lugar de $\bar{A}$ e $\sin (\alpha / 2)$ no lugar de $\bar{B}$, temos que de 3.3$)$ e 3.4

$$
\begin{array}{r}
I=\cos ^{2}(\alpha / 2) d u \otimes d u+\sin ^{2}(\alpha / 2) d v \otimes d v \\
I I=\sin (\alpha / 2) \cos (\alpha / 2) d u \otimes d v-\sin (\alpha / 2) \cos (\alpha / 2) d v \otimes d v .
\end{array}
$$


Consequentemente

$$
\begin{array}{r}
\omega_{1}^{2}=\frac{1}{2} \alpha_{v} d u+\frac{1}{2} \alpha_{u} d v \\
\omega_{1}^{3}=\sin (\alpha / 2) d u \\
\omega_{2}^{3}=-\cos (\alpha / 2) d v
\end{array}
$$

Acabamos de mostrar que na vizinhança de um ponto não-umbílico de uma superfície $X$ em $\mathbb{E}^{3}$ existem coordenadas $(u, v)$ tais que a primeira e segunda formas fundamentais são dadas como em (3.11) e (3.12). Geometricamente o parâmetro $\alpha / 2 \in(0, \pi / 2)$ é ângulo o que a linha de curvatura faz com a linha assintótica (na qual a curvatura normal é nula), de fato, o valor da curvatura normal, $k_{n}$, na direção de um vetor que faz um ângulo $\theta$ com uma direção principal é dada por $k_{n}=k_{1} \cos ^{2}(\theta)+k_{2} \sin ^{2}(\theta)$. De modo que na direção $\alpha / 2$ temos

$$
\begin{aligned}
k_{n}(\alpha / 2) & =k_{1} \cos ^{2}(\alpha / 2)+k_{2} \sin ^{2}(\alpha / 2) \\
& =\tan (\alpha / 2) \cos ^{2}(\alpha / 2)-\cot (\alpha / 2) \sin ^{2}(\alpha / 2) \\
& =0
\end{aligned}
$$

Assim, por definição, concluímos que esta direção é assintótica.

Definição 3.1. Seja $X: U \rightarrow \mathbb{E}^{3}$ uma superfície parametrizada regular. As coordenadas $(u, v)$ como construídas acima, na qual

$$
\begin{array}{r}
I=\cos ^{2}(\alpha / 2) d u \otimes d u+\sin ^{2}(\alpha / 2) d v \otimes d v \\
I I=\sin (\alpha / 2) \cos (\alpha / 2) d u \otimes d v-\sin (\alpha / 2) \cos (\alpha / 2) d v \otimes d v,
\end{array}
$$

são ditas Coordenadas de Tchebyschef. O referencial móvel $\left\{e_{1}, e_{2}, e_{3}\right\}$, no qual $e_{1}$ e $e_{2}$ são direções principais, é dito Referencial Móvel de Tchebyschef.

A construção acima nos permite demonstrar como se dá a clássica relação entre as superfícies de curvatura constante e a equação de sine-Gordon citada no começo desta seção.

Teorema 3.1. A cada superfície parametrizada regular de curvatura constante negativa em $\mathbb{E}^{3}$ está associada uma solução da equação de sine-Gordon;

$$
\alpha_{u u}-\alpha_{v v}=\sin \alpha
$$

Reciprocamente, para cada solução $\alpha \in(0, \pi)$ da equação de sine-Gordon, existe uma superfície de curvatura constante negativa. Além disto esta superfície é única a menos de um movimento rígido em $\mathbb{E}^{3}$. 
Demonstração: Suponha que $X: U \subset \mathbb{R}^{2} \rightarrow \mathbb{E}^{3}$ seja uma superfície com curvatura constante $K=-1$. Conforme vimos sempre podemos supor que, a menos de restrição do aberto $U, X$ está parametrizada por linhas de curvaturas. De modo que existirá uma reparametrização de $X$ por Coordenadas de Tchebyschef e um Referencial Móvel de Tchebyschef. Deste modo, suponha que $X$ esteja parametrizada pelas coordenadas de Techebyschef para o o co-referencial móvel associado ao referencial móvel de Tchebyschef, na qual o co-referencial móvel é dado pelas Equações 3.9 e (3.10) e as formas de conexão dadas por (3.13), 3.14 e(3.15) em que $\alpha \in(0, \pi)$.

Calculemos e Equação de Gauss (2.13),

$$
\begin{aligned}
d \omega_{1}^{2} & =-K \omega^{1} \wedge \omega^{2} \\
d\left(\frac{1}{2} \alpha_{v} d u+\frac{1}{2} \alpha_{u} d v\right) & =(\cos (\alpha / 2) d u) \wedge(\sin (\alpha / 2) d v) \\
\frac{1}{2}\left(\alpha_{u u}-\alpha_{v v}\right) d u \wedge d v & =\cos (\alpha / 2) \sin (\alpha / 2) d u \wedge d v \\
\left(\alpha_{u u}-\alpha_{v v}\right) d u \wedge d v & =\sin (\alpha) d u \wedge d v .
\end{aligned}
$$

Por independência linear de $d u$ e $d v$ a Equação de Gauss é equivalente a equação de sineGordon

$$
\alpha_{u u}-\alpha_{v v}=\sin \alpha
$$

Como a Equação de Gauss é uma condição necessária, temos que $\alpha$ é uma solução para equação de sine-Gordon. E está demonstrada a primeira parte do teorema.

Reciprocamente, seja $0<\alpha<\pi$ uma solução da equação de sine-Gordon. Temos que $\alpha$ define uma expressão para as primeira e segunda formas fundamentais $I$ e $I I$ como nas equações 3.11 e (3.12). Como vimos a equação de Gauss é equivalente a equação de sineGordon, de modo que $\alpha$ satisfaz as equações de compatibilidade. Assim fica definida uma superfície com curvatura Gaussiana constante igual a -1 e esta é única a menos de movimento rígido no espaço Euclidiano.

Este teorema tem uma grande importância, pois permite desenvolver uma das relações entre o campo da Geometria Diferencial e a campo das EDP's, na qual tem consequências na área de Sistemas Dinâmicos Integráveis e Teoria de Sólitons que trabalham com a equação de sin-Gordon. 


\subsubsection{Curvatura constante positiva em $\mathbb{E}^{3}$}

Ao longo deste texto estaremos interessados na Transformação de Bäcklund, ainda a ser definida. Conforme veremos esta é uma transformação geométrica que ocorre entre superfícies com curvatura Gaussiana negativa. De modo para tais fins o estudo de superfícies com curvatura Gaussiana positiva não é de fato essencial. Entretanto é interessante notar que existe uma relação entre as superfícies de curvatura constante positiva e as EDP's. Na verdade é possível relacionar a existência de superfícies de curvatura Gaussiana constante positiva com a equação de sinh-Laplace negativa;

$$
\alpha_{u u}+\alpha_{v v}=-\sinh \alpha
$$

E esta relação se dá usando o mesmo raciocínio empregado para as superfícies de curvatura Gaussiana negativa.

Seja $X: U \subset \mathbb{R}^{2} \rightarrow \mathbb{E}^{3}$ uma superfície parametrizada regular com curvatura Gaussiana, $K$, constante positiva. Como vimos, podemos supor sem perda de generalidade que $K=+1$. Suponha que os pontos de $X$ não sejam umbílicos (para tanto, basta supor que $X$ não está contida em uma esfera). Podemos reconstruir as passagens feitas para o caso da curvatura constante negativa e provar que existe, localmente, Coordenadas de Tchebyschef. Basta tomar, nas Equações (3.7) e (3.8) as curvaturas principais como

$$
\begin{aligned}
& k_{1}= \pm \operatorname{coth}(\alpha / 2), \quad \alpha>0, \\
& k_{2}= \pm \tanh (\alpha / 2) .
\end{aligned}
$$

Consequentemente as expressões do co-referencial móvel de Tchebyschef e as formas de conexão, serão,

$$
\begin{aligned}
\omega^{1} & =\cosh (\alpha / 2) d u, \quad \omega^{2}=\sinh (\alpha / 2) d v, \\
\omega_{1}^{2} & =\frac{1}{2}\left(-\alpha_{v} d u+\alpha_{u} d v\right), \\
\omega_{1}^{3} & =\sinh (\alpha / 2) d u, \quad \omega_{2}^{3}=\cosh (\alpha / 2) d v .
\end{aligned}
$$

Com este co-referencial móvel a Equação de Gauss será equivalente a equação de sinhLaplace negativa. Assim teremos um análogo do Teorema (3.1).

Teorema 3.2. A cada superfície parametrizada regular de curvatura constante positiva em $\mathbb{E}^{3}$ está associada uma solução da equação de sinh-Laplace negativa

$$
\alpha_{u u}+\alpha_{v v}=-\sinh \alpha .
$$


Reciprocamente, para cada solução $\alpha>0$ da equação de sinh-Laplace negativa, existe uma superfície de curvatura constante positiva. Além disto esta superfície é única a menos de um movimento rígido em $\mathbb{E}^{3}$.

Demonstração: A demonstração segue as mesmas linhas da demonstração do Teorema (3.1), com o co-referencial móvel dado pelas Equações (3.20)-(3.22).

\subsection{Superfícies de curvatura constante não nula em $\mathbb{L}^{3}$}

Nesta seção trataremos da relação de todas as superfícies não-degeneradas no espaço de Minkowski, com curvatura Gaussiana constante não nula e suas EDP's naturais. Estas relações seguirão a mesma idéia geral usada na seção anterior, isto é, estabelecer um sistema de coordenadas especial e com ele escrever um referencial móvel cuja Equação de Gauss seja equivalente a uma EDP particular. Porém notamos imediatamente que a teoria apresentada na seção anterior não se aplica diretamente a todas as superfícies no espaço de Minkowski. Por exemplo, para superfícies nas quais a primeira forma fundamental não é positiva definida, pois em geral não está definido curvaturas principais.

Ao longo desta seção consideraremos $\mathbb{X}: U \subset \mathbb{R}^{2} \rightarrow \mathbb{L}^{3}$ uma superfície parametrizada regular não-degenerada de curvatura Gaussiana constante $K$. Sendo a superfície nãodegenerada podemos supor que, a menos de restrição de $U$ que $\mathbb{X}$ é tipo-espaço ou tipo-tempo.

Dividiremos nossa abordagem em dois possíveis caso: superfícies Weingarten-diagonalizáveis e superfícies não Weingarten-diagonalizáveis.

\subsubsection{Superfícies Weingarten-diagonalizáveis}

Suponhamos primeiramente que a superfície é Weingarten-diagonalizável (vide Definição (1.24) e que $\mathbb{X}$ não possui pontos umbílicos (recorde que pelo Teorema 1.5 basta supor que $\mathbb{X}$ não está contidas em planos, planos hiperbólicos ou pseudo-esferas).

Considere $\mathbb{X}(\bar{u}, \bar{v})$ uma superfície no espaço de Minkowski com $(\bar{u}, \bar{v})$ parâmetros de $U$ nos quais as curvas coordenadas são linhas de curvatura. Seja $\left\{e_{1}, e_{2}, e_{3}\right\}$ um referencial móvel tal que $e_{1}$ e $e_{2}$ estejam nas direções principais, isto é, $d N_{p}\left(e_{i}\right)=-k_{i} e_{i}$, e $\left\{\omega^{1}, \omega^{2}\right\}$ o co-referencial móvel associado. 
Analogamente ao que foi para superfícies no espaço Euclidiano, vamos expressar o coreferencial móvel e suas formas de conexão em termos da base $\{d \bar{u}, d \bar{v}\}$ de modo que a decomposição nesta base envolverá as curvaturas principais $k_{1}$ e $k_{2}$. A partir disto usaremos as equações de estrutura para obter a EDP natural.

Expressando o co-referencial na base $\{d \bar{u}, d \bar{v}\} ; \operatorname{como} d \mathbb{X}=\mathbb{X}_{\bar{u}} d \bar{u}+\mathbb{X}_{\bar{v}} d \bar{v}$, temos

$$
\begin{gathered}
\omega^{1}=\left\langle d \mathbb{X}, e_{1}\right\rangle=\left\langle\mathbb{X}_{\bar{u}}, e_{1}\right\rangle d \bar{u}:=\bar{A} d \bar{u} \\
\omega^{2}=\left\langle d \mathbb{X}, e_{2}\right\rangle=\left\langle\mathbb{X}_{\bar{v}}, e_{2}\right\rangle d \bar{v}:=\bar{B} d \bar{v}
\end{gathered}
$$

Isto é $\omega^{1}$ e $\omega^{2}$ são proporcionais aos elementos da base $d \bar{u}$ e $d \bar{v}$, respectivamente, pelas funções $\bar{A}(\bar{u}, \bar{v})$ e $\bar{B}(\bar{u}, \bar{v})$ definidas em $U$. Logo a tripla de 1-formas diferenciais $d \mathbb{X}$ é expressa por

$$
d \mathbb{X}=\epsilon_{1} \omega^{1} e_{1}+\epsilon_{2} \omega^{2} e_{2}=\epsilon_{1} \bar{A} e_{1} d \bar{u}+\epsilon_{2} \bar{B} e_{2} d \bar{v}
$$

Consequentemente a primeira forma fundamental $I$ é dada por

$$
I=\langle d \mathbb{X}, d \mathbb{X}\rangle=\epsilon_{1} \bar{A}^{2} d \bar{u} \otimes d \bar{u}+\epsilon_{2} \bar{B}^{2} d \bar{v} \otimes d \bar{v}
$$

Como $\mathbb{X}_{\bar{u}}=\epsilon_{1}\left\langle\mathbb{X}_{\bar{u}}, e_{1}\right\rangle e_{1}+\epsilon_{2}\left\langle\mathbb{X}_{\bar{u}}, e_{2}\right\rangle e_{2}=\epsilon_{1} \bar{A} e_{1}$ e analogamente $\mathbb{X}_{\bar{v}}=\epsilon_{2} \bar{B} e_{2}$ temos que,

$$
\begin{aligned}
& N_{\bar{u}}=d N\left(\mathbb{X}_{u}\right)=d N\left(\epsilon_{1} \bar{A} e_{1}\right)=-\epsilon_{1} \bar{A} k_{1} e_{1} \\
& N_{\bar{v}}=d N\left(\mathbb{X}_{v}\right)=d N\left(\epsilon_{2} \bar{B} e_{2}\right)=-\epsilon_{2} \bar{B} k_{2} e_{2}
\end{aligned}
$$

Logo a diferencial de $N$ é escrita por

$$
d N=N_{\bar{u}} d \bar{u}+N_{\bar{v}} d \bar{v}=-\epsilon_{1} \bar{A} k_{1} d \bar{u} e_{1}-\epsilon_{2} \bar{B} k_{2} d \bar{v} e_{2} .
$$

Consequentemente a segunda forma fundamental $I I$ é dada por

$$
\begin{aligned}
I I & =\langle-d N, d \mathbb{X}\rangle \\
& =\left\langle\epsilon_{1} \bar{A} k_{1} d \bar{u} e_{1}+\epsilon_{2} \bar{B} k_{2} d \bar{v} e_{2}, \epsilon_{1} \bar{A} e_{1} d \bar{u}+\epsilon_{2} \bar{B} e_{2} d \bar{v}\right\rangle \\
& =\epsilon_{1} \bar{A}^{2} k_{1} d \bar{u} \otimes d \bar{u}+\epsilon_{2} \bar{B}^{2} k_{2} d \bar{v} \otimes d \bar{v} .
\end{aligned}
$$

Por outro lado, em termos das formas de conexão

$$
\begin{aligned}
I I & =\langle-d N, d \mathbb{X}\rangle=\left\langle-d e_{3}, d \mathbb{X}\right\rangle \\
& =\left\langle-\epsilon_{1} \omega_{3}^{1} e_{1}-\epsilon_{2} \omega_{3}^{2} e_{2}, \epsilon_{1} \omega^{1} e_{1}+\epsilon_{2} \omega^{2} e_{2}\right\rangle \\
& =-\epsilon_{1} \omega_{3}^{1} \otimes \omega^{1}-\epsilon_{2} \omega_{3}^{2} \otimes \omega^{2} .
\end{aligned}
$$


Comparando as duas expressões para a segunda forma fundamental, concluímos que

$$
\begin{array}{ll}
\omega_{3}^{1}=-k_{1} \bar{A} d \bar{u} & \left(=-k_{1} \omega^{1}\right), \\
\omega_{3}^{2}=-k_{2} \bar{B} d \bar{v} & \left(=-k_{2} \omega^{2}\right) .
\end{array}
$$

Assim temos a expressão das formas de conexão $\omega_{3}^{i}$ na base $\{d \bar{u}, d \bar{v}\}$.

Por último vamos usar as equações de estrutura (2.17) e (2.18) juntamente com a Proposição 2.9 para expressar a forma de conexão $\omega_{1}^{2}$ nesta base. Para tanto, basta notar que a ansatz;

$$
\omega_{1}^{2}=-\epsilon_{2} \frac{\bar{A}_{\bar{v}}}{\bar{B}} d \bar{u}+\epsilon_{1} \frac{\bar{B}_{\bar{u}}}{\bar{A}} d \bar{v},
$$

satisfaz a Equação 2.17): $d \omega^{1}=\epsilon_{2} \omega^{2} \wedge \omega_{2}^{1}$ e a Equação 2.18): $d \omega^{2}=\epsilon_{1} \omega^{1} \wedge \omega_{1}^{2}$. De modo que temos o co-referencial móvel e as formas de conexão expressas na base $\{d \bar{u}, d \bar{v}\}$. Analogamente ao que feito na seção anterior para o caso Euclidiano, as Equações de Estrutura 2.21) e 2.22 implicam em

$$
\begin{aligned}
& \left(k_{1}\right)_{\bar{v}} \bar{A}+\left(k_{1}-k_{2}\right) \bar{A}_{\bar{v}}=0, \\
& \left(k_{2}\right)_{\bar{u}} \bar{B}+\left(k_{2}-k_{1}\right) \bar{B}_{\bar{u}}=0 .
\end{aligned}
$$

A partir de agora analisemos cada possível caso que ocorre para uma superfície nãodegenerada no espaço de Minkowski. Teremos ao todo quatro casos.

\section{Caso I: Superfícies Tipo-Espaço com Curvatura constante positiva}

Neste caso, $\epsilon_{1}=\epsilon_{2}=1$ e $\epsilon_{3}=-1$ e podemos supor que $K=1$. Desse modo o discriminante do polinômio característico de $\mathbb{X}$, dado na Equação 1.22 é estritamente positivo,

$$
\triangle=4\left(H^{2}+1\right)>0
$$

e sempre existirá duas curvaturas principais distintas $k_{1}$ e $k_{2}$. Pela relação $K=\epsilon k_{1} k_{2}$, podemos supor que $k_{2}<0<k_{1}$. Deste modo existe $\alpha \in(0, \pi)$ tal que,

$$
\begin{gathered}
k_{1}=\tan (\alpha / 2), \\
k_{2}=-\cot (\alpha / 2) .
\end{gathered}
$$

Deste modo que as Equações 3.24 e (3.25) serão equivalentes a, respectivamente

$$
\begin{gathered}
\bar{A}=e^{a(\bar{u})} \cos (\alpha / 2) \\
\bar{B}=e^{b(\bar{u})} \sin (\alpha / 2)
\end{gathered}
$$


para algum par de funções diferenciáveis $a(\bar{u})$ e $b(\bar{v})$. Deste modo, reparametrizamos $\mathbb{X}$ via a mudança de coordenadas $h$, dada por

$$
(\bar{u}, \bar{v}) \rightarrow(u, v) ; \quad(u, v)=h(\bar{u}, \bar{v})=\left(\int^{\bar{u}} e^{a(\xi)} d \xi, \int^{\bar{v}} e^{b(\xi)} d \xi\right)
$$

Dizemos que estas novas coordenadas $(u, v)$ são Coordenadas de Tchebyschef I e o referencial móvel $\left\{e_{1}, e_{2}, e_{3}\right\}$, no qual $e_{1}$ e $e_{2}$ são direções principais, é dito Referencial Móvel de Tchebyschef I. Neste referencial, o co-referencial móvel é dado por;

$$
\begin{aligned}
\omega^{1} & =\cos (\alpha / 2) d u, \\
\omega^{2} & =\sin (\alpha / 2) d v,
\end{aligned}
$$

e o co-referencial móvel é dado por pelas equações

$$
\begin{aligned}
\omega_{1}^{2} & =\frac{1}{2} \alpha_{v} d u+\frac{1}{2} \alpha_{u} d v \\
\omega_{1}^{3} & =\sin (\alpha / 2) d u \\
\omega_{2}^{3} & =-\cos (\alpha / 2) d v
\end{aligned}
$$

Além disso, a primeira e a segunda forma fundamental são dadas por

$$
\begin{aligned}
I & =\cos ^{2}(\alpha / 2) d u \otimes d u+\sin ^{2}(\alpha / 2) d v \otimes d v \\
I I & =\cos (\alpha / 2) \sin (\alpha / 2)(d u \otimes d u-d v \otimes d v) .
\end{aligned}
$$

Da mesma forma que fizemos no caso Euclidiano, a Equação de Gauss 2.24 $d \omega_{1}^{2}=$ $-K \omega^{1} \wedge \omega^{2}$ será equivalente a equação de sine-Gordon

$$
\alpha_{u u}-\alpha_{v v}=\sin \alpha
$$

Deste modo demonstramos o seguinte resultado.

Teorema 3.3. Existe uma relação biunívoca entre as soluções da equação de sine-Gordon e a existência, a menos de movimento rígido no espaço de Minkowski, de superfícies tipo-espaço com curvatura Gaussiana constante positiva.

\section{Caso II: Superfícies Tipo-Espaço com Curvatura constante negativa}

Suponha que $\mathbb{X}$ seja uma superfície tipo-espaço com curvatura Gaussiana constante negativa. Assim, $\epsilon_{1}=\epsilon_{2}=1, \epsilon_{3}=-1$ e $K=-1$. Note que o discriminante do polinômio característico de $\mathbb{X}, \triangle=4\left(H^{2}-1\right) \geqslant 0$ de modo que nem sempre $\mathbb{X}$ será uma superfície não 
umbílica. Deste modo suponha que $\mathbb{X}$ não admita pontos umbílicos (ou use o Teorema (1.5) e suponha que $\mathbb{X}$ não está contida em um plano ou em m plano hiperbólico).

Assim existirão duas curvaturas principais distintas $k_{1}$ e $k_{2}$. Pela relação $K=\epsilon k_{1} k_{2}$, podemos supor que $k_{2}<k_{1}$ são ambos números positivos ou negativos. Deste modo existe $\alpha \in(0,+\infty)$ tal que,

$$
\begin{aligned}
& k_{1}=\tau \operatorname{coth}(\alpha / 2), \\
& k_{2}=\tau \tanh (\alpha / 2),
\end{aligned}
$$

$\operatorname{com} \tau=\operatorname{sinal}\left(k_{1}\right)=\operatorname{sinal}\left(k_{2}\right)$. E as Equações 3.24 e 3.25) serão equivalentes a, respectivamente,

$$
\begin{aligned}
& \bar{A}=e^{a(\bar{u})} \sinh (\alpha / 2) \\
& \bar{B}=e^{b(\bar{u})} \cosh (\alpha / 2)
\end{aligned}
$$

para alguma par de funções diferenciáveis $a(\bar{u})$ e $b(\bar{v})$.

Reparametrizando $\mathbb{X}$ por $h$, mudança de coordenadas dada por

$$
(\bar{u}, \bar{v}) \rightarrow(u, v) ; \quad(u, v)=h(\bar{u}, \bar{v})=\left(\int^{\bar{u}} e^{a(\xi)} d \xi, \int^{\bar{v}} e^{b(\xi)} d \xi\right)
$$

temos novas coordenadas $(u, v)$ que denotaremos por Coordenadas de Tchebyschef II, e o referencial móvel $\left\{e_{1}, e_{2}, e_{3}\right\}$, no qual $e_{1}$ e $e_{2}$ são direções principais, será dito Referencial Móvel de Tchebyschef II, e neste referencial, o co-referencial móvel é dado por

$$
\begin{aligned}
& \omega^{1}=\sinh (\alpha / 2) d u \\
& \omega^{2}=\cosh (\alpha / 2) d v
\end{aligned}
$$

e o co-referencial móvel é dado por pelas equações

$$
\begin{aligned}
\omega_{1}^{2} & =-\frac{1}{2} \alpha_{v} d u+\frac{1}{2} \alpha_{u} d v, \\
\omega_{1}^{3} & =\cosh (\alpha / 2) d u, \\
\omega_{2}^{3} & =\sinh (\alpha / 2) d v .
\end{aligned}
$$

e a primeira e a segunda forma fundamental

$$
\begin{aligned}
I & =\cosh ^{2}(\alpha / 2) d u \otimes d u-\sinh ^{2}(\alpha / 2) d v \otimes d v \\
I I & =\cos (\alpha / 2) \sin (\alpha / 2)(d u \otimes d u-d v \otimes d v) .
\end{aligned}
$$


A Equação de Gauss será equivalente a equação de sinh-Gordon;

$$
\alpha_{u u}-\alpha_{v v}=\sinh \alpha
$$

Logo demonstramos o seguinte resultado.

Teorema 3.4. Existe uma relação biunívoca entre as soluções da equação de sinh-Gordon com a existência, a menos de um movimento rígido no espaço Minkowski, de superfícies tipotempo que assumem duas curvaturas principais distintas de curvatura Gaussiana constante positiva.

\section{Caso III: Superfícies Tipo-Tempo com Curvatura constante negativa}

Neste caso, $\epsilon_{1}=1, \epsilon_{2}=-1, \epsilon_{3}=1$ e $K=-1$. De modo que o discriminante do polinômio característico de $\mathbb{X}, \triangle=4\left(H^{2}+1\right)>0$, é estritamente positivo. Assim sempre existirão duas curvaturas principais distintas $k_{1}$ e $k_{2}$. Pela relação $K=\epsilon k_{1} k_{2}$, podemos supor que $k_{2}<0<k_{1}$. Deste modo existe $\alpha \in(0, \pi)$ tal que,

$$
\begin{array}{r}
k_{1}=\tan (\alpha / 2) \\
k_{2}=-\cot (\alpha / 2) .
\end{array}
$$

Deste modo definimos uma mudança de parâmetros inteiramente análoga ao Caso I, e também dizemos que estas novas coordenadas $(u, v)$ são Coordenadas de Tchebyschef III, e o referencial móvel $\left\{e_{1}, e_{2}, e_{3}\right\}$, no qual $e_{1}$ e $e_{2}$ são direções principais, é dito Referencial Móvel de Tchebyschef III, e neste referencial, o co-referencial móvel é dado por;

$$
\begin{aligned}
\omega^{1} & =\cos (\alpha / 2) d u, \\
\omega^{2} & =\sin (\alpha / 2) d v,
\end{aligned}
$$

e o co-referencial móvel é dado por pelas equações

$$
\begin{aligned}
\omega_{1}^{2} & =\frac{1}{2} \alpha_{v} d u+\frac{1}{2} \alpha_{u} d v, \\
\omega_{1}^{3} & =\sin (\alpha / 2) d u \\
\omega_{2}^{3} & =\cos (\alpha / 2) d v,
\end{aligned}
$$

e a primeira e e segunda forma fundamental

$$
\begin{aligned}
I & =\cos ^{2}(\alpha / 2) d u \otimes d u-\sin ^{2}(\alpha / 2) d v \otimes d v \\
I I & =\cos (\alpha / 2) \sin (\alpha / 2)(d u \otimes d u+d v \otimes d v)
\end{aligned}
$$


E neste referencial a Equação de Gauss é equivalente a equação de Sin-Laplace;

$$
\alpha_{u u}-\alpha_{v v}=\sin \alpha .
$$

O que acarreta na demonstração do seguinte teorema.

Teorema 3.5. Existe uma relação biunívoca entre as soluções da equação de sine-Laplace com a existência, a menos de um movimento rígido no espaço Minkowski, de superfícies tipo-tempo com curvatura Gaussiana constante negativa.

\section{Caso IV: Superfícies Tipo-Tempo com Curvatura constante positiva e duas cur- vaturas principais reais distintas}

Suponha que $\mathbb{X}$ seja uma superfície tipo-tempo com curvatura Gaussiana constante positiva. Note que em geral o discriminante do polinômio característico de $\mathbb{X}, \triangle=4\left(H^{2}-1\right)$ não tem sinal definido. Para este caso, suponha que $\triangle>0$ em todos os pontos, isto é que em cada ponto $\mathbb{X}$ possua duas curvaturas principais distintas.

Seja então $k_{1} \neq k_{2}$ as duas curvaturas principais. Pela relação $K=\epsilon k_{1} k_{2}$, podemos supor que $k_{2}<k_{1}$ e são ambos números positivos ou negativos. Deste modo existe $\alpha \in(0,+\infty)$ tal que,

$$
\begin{aligned}
& k_{1}=\tau \operatorname{coth}(\alpha / 2), \\
& k_{2}=\tau \tanh (\alpha / 2),
\end{aligned}
$$

em que $\tau= \pm 1$.

Perceba que estas funções definem a mesma reparametrização feita no Caso II, e estas novas coordenadas $(u, v)$ também serão denotadas por Coordenadas de Tchebyschef IV, e o referencial móvel $\left\{e_{1}, e_{2}, e_{3}\right\}$, no qual $e_{1}$ e $e_{2}$ são direções principais, será dito Referencial Móvel de Tchebyschef IV, e neste referencial, o co-referencial móvel é dado por,

$$
\begin{aligned}
& \omega^{1}=\sinh (\alpha / 2) d u, \\
& \omega^{2}=\cosh (\alpha / 2) d v,
\end{aligned}
$$

e o co-referencial móvel é dado por pelas equações,

$$
\begin{aligned}
\omega_{1}^{2} & =-\frac{1}{2} \alpha_{v} / 2 d u+\frac{1}{2} \alpha_{u} / 2 d v, \\
\omega_{1}^{3} & =\cosh (\alpha / 2) d u, \\
\omega_{2}^{3} & =\sinh (\alpha / 2) d v,
\end{aligned}
$$


e a primeira e a segunda forma fundamental

$$
\begin{aligned}
I & =\cosh ^{2}(\alpha / 2) d u \otimes d u+\sinh ^{2}(\alpha / 2) d v \otimes d v \\
I I & =\cos (\alpha / 2) \sin (\alpha / 2)(d u \otimes d u+d v \otimes d v)
\end{aligned}
$$

Deste modo a Equação de Gauss será equivalente a equação de sinh-Gordon;

$$
\alpha_{u u}-\alpha_{v v}=-\sinh \alpha .
$$

De modo que demonstramos o seguinte resultado.

Teorema 3.6. Existe uma relação biunívoca entre as soluções da equação de sinh-Gordon Negativa com a existência, a menos de um movimento rígido no espaço Minkowski, de superfícies tipo-tempo com curvatura Gaussiana constante positiva com duas direções principais reais.

\subsubsection{Superfícies não Weingarten-diagonalizáveis}

Suponhamos agora que a superfície não é Weingarten-diagonalizável. Devemos ter que $\mathbb{X}$ é uma superfície tipo-tempo cujo discriminante do polinômio característico ou é nulo ou estritamente negativo, e assim teremos dois casos.

\section{Caso V: Superfícies Tipo-Tempo com Curvatura constante positiva e uma curva- tura principal real}

Suponha que $\mathbb{X}$ seja uma superfície não Weingarten-diagonalizável com o discriminante do polinômio característico $\triangle=0$. Neste caso foi mostrado pela Louise V. MacNertney em 1980 [15] que a existência de superfícies com estas características é condicionada a existência de soluções da equação de Liouville $\alpha_{u v}=\frac{1}{2} e^{\alpha}$.

Seguindo então [15] vamos mostrar como se dá tal relação.

Se $\triangle=0$ então o operador forma $S_{q}$ de $\mathbb{X}$ admite um auto-valor. Se o auto-espaço correspondente tem dimensão dois então $\mathbb{X}$ é umbílica (e ainda estará contida em alguma superfície dada no Teorema 1.5. Deste modo, supondo que $\mathbb{X}$ não é umbílica temos que o auto-espaço correspondente tem dimensão 1. Vamos mostrar que este será um subespaço tipo-luz.

Lema 3.1. Seja $\mathbb{X}$ uma superfície no espaço de Minkowski tal que o operador $S_{q}$ admita um único auto-valor com auto-espaço correspondente de dimensão 1. Então o auto-espaço é um subespaço tipo-luz. 
Demonstração: Por definição se $\lambda$ é um auto-valor de $S_{q}$ então o auto-espaço $W_{\lambda}$ correspondente a $\lambda$ é o subespaço vetorial de $T_{q} \mathbb{X}$ que contém todos auto-vetores de $S_{q}$ correspondentes a $\lambda$. Seja $w \in T_{q} \mathbb{X}$ tal que $w \perp v$ com $v \in W_{\lambda}$, isto é $\langle w, v\rangle=0$. Como $S_{q}$ é auto-adjunta, temos que

$$
\left\langle S_{q}(w), v\right\rangle=\left\langle w, S_{q}(v)\right\rangle=\langle w, \lambda v\rangle=\lambda\langle v, w\rangle=0
$$

Consequentemente, $S_{q}(w) \in W_{\lambda}^{\perp}$.

Suponhamos por absurdo que $v$, auto-vetor de $S_{q}$ não é um vetor tipo-luz. Dividindo $v$ por sua norma podemos supor que $v$ está normalizado, assim tomando $w \in W_{\lambda}^{\perp}$ normalizado podemos construir uma base $\{w, v\}$ de $T_{q} \mathbb{X}$, donde $S_{q}(w)=\mu w+\xi v$ é escrito nesta base para algum par de números reais $\mu$ e $\xi$. Conforme mostramos $S_{q}(w) \in W_{\lambda}^{\perp}$ e segue que $\xi=0, \operatorname{logo}$ $S_{q}(w)=\mu w$ e $\operatorname{assim} w$ é auto-vetor de $S_{q}$ o que é um absurdo. De modo que $v$ é um vetor tipo-luz, e consequentemente $W_{\lambda}$ é um subespaço tipo-luz.

Teorema 3.7. Seja $\mathbb{X}: U \subset \mathbb{R}^{2} \rightarrow \mathbb{L}^{3}$ uma superfície tipo-tempo com curvatura Gaussiana $K=1$ tal que o operador de Weingarten $S_{q}$ tem um auto-valor $\lambda$ de multiplicidade dois cujo auto-espaço correspondente tem dimensão 1. Então,

1. $\lambda=1$ (ou $\lambda=-1)$ e traçoS $S_{q}=2($ ou $=-2)$,

2. dado $q \in U$ existe uma vizinhança de $V_{q}$ de $q$ em $U$, tal que para cada $\bar{q} \in V_{q}$ existem $u, v \in T_{\bar{q}} \mathbb{X}$ vetores linearmente independentes, tipo-luz com $v$ um auto-vetor de $S_{q}$.

Antes de demonstrá-lo lembremos do famoso resultado da Álgebra Linear, o Teorema de Cayley-Hamilton ${ }^{1}$. Seja $V$ um espaço vetorial real de dimensão finita e $\mathbb{R}[z]$ o conjunto de todos os polinômios com coeficientes em $\mathbb{R}$ na incógnita $z$. Dados $q(z)=\sum_{j=0}^{n} \alpha_{j} z^{j} \in \mathbb{R}[z]$ um polinômio e $T: V \rightarrow V$ um operador linear em $V$, fica bem definido o operador em $V$ dado por

$$
q(T)=\alpha_{n} T^{n}+\alpha_{n-1} T^{n-1}+\ldots+\alpha_{1} T+\alpha_{0} I
$$

em que $T^{j}=\underbrace{T \circ \ldots \circ T}_{j \text {-vezes }}$ e $T^{0}=I$ é a identidade de $V$ em $V$. O Teorema de Cayley-Hamilton nos garante que, dados $T: V \rightarrow V$, com polinômio característico $p(\lambda)$, o polinômio $p(T)$ é um operador nulo sobre $V$, isto é, $\forall v \in V$ tem-se que $p(T)(v)=0 \in V$. Vamos agora a

\footnotetext{
${ }^{1}$ Encontrado nos livros textos de Álgebra Linear, como por exemplo 13 . página 89.
} 
demonstração do Teorema (3.7);

\section{Demonstração (do Teorema (3.7)):}

1. Pelo Lema (3.1) seja $v_{q} \in T_{q} \mathbb{X}$ um vetor tipo-luz em que $S_{q}\left(v_{q}\right)=\lambda(q) v_{q}$. Tome $u_{q} \in T_{q} \mathbb{X}$ outro vetor tipo-luz linearmente independente de $v_{q}$, que sempre existe via Proposição 1.5 item 3 juntamente com o fato de $\mathbb{X}$ ser tipo-tempo. De modo que o conjunto $\left\{u_{q}, v_{q}\right\}$ forma uma base de vetores tipo-luz de $T_{q} \mathbb{X}$, e como são linearmente independentes pela Proposição 1.4 podemos supor, re-escalando os vetores $u_{q}$ e $v_{q}$ se necessário, que $\left\langle v_{q}, u_{q}\right\rangle=1$.

Escrevendo $S_{q}\left(u_{q}\right)=\xi u_{q}+\mu v_{q}$ nesta base, temos,

$$
\left\langle S_{q}\left(u_{q}\right), v_{q}\right\rangle=\left\langle\xi u_{q}+\mu v_{q}, v_{q}\right\rangle=\xi
$$

Por outro lado, como $S_{q}$ é auto-adjunta, segue que

$$
\left\langle S_{q}\left(u_{q}\right), v_{q}\right\rangle=\left\langle u_{q}, S_{q}\left(v_{q}\right)\right\rangle=\left\langle u_{q}, \lambda v_{q}\right\rangle=\lambda .
$$

Deste modo, $S_{q}\left(u_{q}\right)=\lambda u_{q}+\mu v_{q}$ e assim a matriz representativa de $S_{q}$ nesta base é dada por

$$
S_{q} \doteq\left(\begin{array}{cc}
\lambda & 0 \\
\mu & \lambda
\end{array}\right) \text {. }
$$

Usando a hipótese de $K=1$, decorre da definição de curvatura $K(q)=\epsilon \operatorname{det} S_{q} \operatorname{com}$ $\epsilon=1$, que $\lambda^{2}=1$ e traço $S_{q}=2 \lambda$. Como $U$ é conexo e $K$ é contínua temos que $\lambda(q)=1$ $\mathrm{ou}=-1$, e assim o resultado do primeiro item segue.

2. Suponha sem perda de generalidade que $\lambda=1$ é o auto-valor de $S_{q}$. Como o autoespaço tem dimensão 1 , existe $u_{q} \in T_{q} \mathbb{X}$ um vetor tal que $S_{q}\left(u_{q}\right) \neq u_{q}$, seja então o vetor $v_{q}:=S_{q}\left(u_{q}\right)-u_{q} \neq 0$ não nulo de $T_{q} \mathbb{X}$. Aplicando $S_{q}$ no vetor não nulo $v_{q}$, temos por linearidade de $S_{q}$ que $S_{q}\left(v_{q}\right)=S_{q}^{2}\left(u_{q}\right)-S_{q}\left(u_{q}\right)$, consequentemente, pela definição de $v_{q}$, segue que

$$
\begin{aligned}
S_{q}\left(v_{q}\right)-v_{q} & =S_{q}^{2}\left(u_{q}\right)-S_{q}\left(u_{q}\right)-\left(S_{q}\left(u_{q}\right)-u_{q}\right) \\
& =S_{q}^{2}\left(u_{q}\right)-2 S_{q}\left(u_{q}\right)-u_{q} \\
& =\left(S_{q}^{2}-2 S_{q}+I\right)\left(u_{q}\right) .
\end{aligned}
$$


Por outro lado, recordamos que o polinômio caractersítico de $\mathbb{X}$ dado na expressão (1.21), é

$$
P(\lambda)=\lambda^{2}-2 \epsilon H \lambda+\epsilon K=\lambda^{2}-\operatorname{traço} S_{q} \lambda+\operatorname{det} S_{q}=\lambda^{2}-2 S_{q}+1
$$

Assim pelo Teorema de Cayley-Halmilton, o operador $p\left(S_{q}\right)$ é o operador nulo de $T_{q} \mathbb{X}$. Como o lado direito de (3.46) é precisamente $p\left(S_{q}\right)\left(u_{q}\right)$, temos que $S_{q}\left(v_{q}\right)-v_{q}=0$.

Consequentemente $S_{q}\left(v_{q}\right)=\lambda v_{q}$ e assim $v_{q}$ é um auto-vetor de $S_{q}$. Novamente pelo Lema (3.1) devemos ter que $v_{q}$ é um vetor tipo-luz, e analogamente ao item anterior o vetor $u_{q}$ também será tipo-luz, de modo que em $T_{q} \mathbb{X}$ eles formam uma base de vetores tipo-luz com um deles correspondendo a um auto-vetor de $S_{q}$.

Por último, como $S_{q}$ depende diferenciavelmente do ponto $q$ podemos tomar uma vizinhança $V_{q}$ de $q$ em $U$ tal que para cada $\bar{q} \in V_{q}$ temos que $S_{\bar{q}}\left(u_{\bar{q}}\right)-u_{\bar{q}} \neq 0$ e repetir o argumento acima. Deste modo, nesta vizinhança os vetores $u_{\bar{q}}$ e $v_{\bar{q}}$ satisfarão a tese e o teorema está demonstrado.

A importância deste teorema reside no seguinte corolário.

Corolário 3.1. Seja $\mathbb{X}: U \subset \mathbb{R}^{2} \rightarrow \mathbb{L}^{3}$ uma superfície nas hipóteses do Teorema 3.7). Então em cada $q \in U$ existe uma reparametrização de $\mathbb{X}$ tal que em uma vizinhança de $q$ os vetores $u_{\bar{q}}$ e $v_{\bar{q}}$, satisfazendo o item 2 do Teorema (3.7), são os vetores coordenados.

Demonstração: Pelo Teorema (3.7), dado $q \in U$ temos uma vizinhança deste ponto na qual fica definido um campo de vetores $u_{\bar{q}}$ e $v_{\bar{q}}$ diferenciáveis linearmente independentes, de modo que tal reparametrização será encontrada obtendo as curvas integrais deste campo, que localmente sempre existem. Este é um fato conhecido e se encontra nos livros de geometria diferencial, como por exemplo o Teorema 3.3.4, página 95 de [2]: Sejam dois campos de direções linearmente independentes definidas em um aberto da superfície. Então cada ponto desta vizinhança está contido em uma vizinhança parametrizada cujas curvas coordenadas são linhas integrais destes campos.

Definição 3.2. Seja $\mathbb{X}(u, v)$ uma superfície parametrizada regular em $\mathbb{L}^{3}$. Dizemos que as coordenadas $(u, v)$ são Coordenadas Nulas de McNertney desde que os vetores coordenados $\mathbb{X}_{u}$ e $\mathbb{X}_{v}$ satisfaçam as condições 2) do Teorema (3.7). Isto é, são vetores dois vetores 
linearmente independentes tipo-luz, $\left\langle\mathbb{X}_{u}, \mathbb{X}_{v}\right\rangle=\mu>0$ e $\mathbb{X}_{v}$ é um auto-vetor do operador Weingarten.

Com esta definição o Corolário (3.1) pode ser reescrito por: Se $\mathbb{X}$ é uma superfície tipotempo com curvatura Gaussiana $K=1$ e não Weingarten-diagonalizável com uma única direção principal, então localmente $\mathbb{X}$ admite coordenadas de McNertney.

Suponhamos que $\mathbb{X}(u, v)$ nestas condições esteja parametrizada por coordenadas de McNertney. É imediato verificar que nas coordenadas de McNertney os coeficientes da primeira forma são, respectivamente,

$$
\begin{array}{r}
E=\left\langle\mathbb{X}_{u}, \mathbb{X}_{u}\right\rangle=0 \\
F=\left\langle\mathbb{X}_{u}, \mathbb{X}_{v}\right\rangle=\mu>0 \\
G=\left\langle\mathbb{X}_{v}, \mathbb{X}_{v}\right\rangle=0
\end{array}
$$

Definindo em cada ponto $q, e_{1}=\mathbb{X}_{u}, e_{2}=\mathbb{X}_{v}$ e $e_{3}=N$ com $N$ normal a $\mathbb{X}$, temos que $\left\{e_{1}, e_{2}, e_{3}\right\}$ formam um Referencial Nulo adaptado a $\mathbb{X}$ (conforme a seção 2.2.3)). Assim, $e_{1}$ e $e_{2}$ são vetores tipo-luz linearmente independentes com $\left\langle e_{1}, e_{2}\right\rangle=\mu>0$. Denotemos

$$
\mu(u, v)=e^{2 \alpha(u, v)}>0
$$

e nomeemos tal Referencial Nulo por Referencial Móvel Nulo de McNertney.

Neste referencial, temos que o co-referencial móvel, é via Equações (2.28), expresso por 2.29

$$
\begin{aligned}
& \omega^{1}=\mu^{-1}\left\langle d \mathbb{X}, e_{2}\right\rangle=e^{-2 \alpha}\left\langle\mathbb{X}_{u} d u+\mathbb{X}_{v} d v, e_{2}\right\rangle=e^{-2 \alpha}\left\langle\mathbb{X}_{v}, e_{1}\right\rangle d u=d u \\
& \omega^{2}=\mu^{-1}\left\langle d \mathbb{X}, e_{1}\right\rangle=e^{-2 \alpha}\left\langle\mathbb{X}_{u} d u+\mathbb{X}_{v} d v, e_{2}\right\rangle=e^{-2 \alpha}\left\langle\mathbb{X}_{u}, e_{2}\right\rangle d v=d v
\end{aligned}
$$

A forma de conexão $\omega_{2}^{2}$ é, via Equação 2.31, dada por

$$
\begin{aligned}
\omega_{2}^{2} & =\mu^{-1}\left\langle d e_{2}, e_{1}\right\rangle \\
& =e^{-2 \alpha}\left\langle\left(e_{2}\right)_{u} d u+\left(e_{2}\right)_{v} d v, e_{1}\right\rangle \\
& =e^{-2 \alpha}\left\langle\mathbb{X}_{v u} d u+\mathbb{X}_{v v} d v, e_{1}\right\rangle \\
& =e^{-2 \alpha}\left\langle\mathbb{X}_{v u}, \mathbb{X}_{u}\right\rangle d u+e^{-2 \alpha}\left\langle\mathbb{X}_{v v}, \mathbb{X}_{u}\right\rangle d v
\end{aligned}
$$

Tomando derivadas parciais de $E, F, G$, verificamos que

$$
\begin{array}{r}
\left\langle\mathbb{X}_{v u}, \mathbb{X}_{u}\right\rangle=\frac{1}{2} E_{v} \\
\left\langle\mathbb{X}_{v v}, \mathbb{X}_{u}\right\rangle=F_{v}-\frac{1}{2} G_{u}
\end{array}
$$


Usando as Equações 3.47)-3.49, temos que

$$
\begin{array}{r}
\left\langle\mathbb{X}_{v u}, \mathbb{X}_{u}\right\rangle=0 \\
\left\langle\mathbb{X}_{v v}, \mathbb{X}_{u}\right\rangle=2 \alpha_{v} e^{2 \alpha}
\end{array}
$$

Imediatamente, a forma $\omega_{2}^{2}$ é dada por

$$
\omega_{2}^{2}=e^{-2 \alpha} 2 e^{2 \alpha} \alpha_{v} d v=2 \alpha_{v} d v
$$

Consequentemente sua derivada exterior é,

$$
\begin{aligned}
d \omega_{2}^{2} & =\left(2 \alpha_{v}\right)_{u} d u \wedge d v+\left(2 \alpha_{v}\right)_{v} d v \wedge d v \\
& =2 \alpha_{v u} d u \wedge d v \\
& =2 \alpha_{v u} \omega^{1} \wedge \omega^{2}
\end{aligned}
$$

Por último, comparando esta expressão com Equação de Gauss 2.44 para um referencial nulo, $d \omega_{2}^{2}=-e^{2 \alpha} \omega^{1} \wedge \omega^{2}$, temos que função $\alpha$ deve satisfazer a equação de Liouville

$$
\alpha_{u v}=-\frac{1}{2} e^{2 \alpha}
$$

Assim temos o seguinte Teorema.

Teorema 3.8. Existe uma relação biunívoca entre as soluções da equação de Liouville com a existência, a menos de um movimento rígido no espaço Minkowski, de superfícies tipo-tempo com curvatura Gaussiana constante positiva com uma única direção principal real.

\section{Caso VI: Superfícies Tipo-Tempo com Curvatura constante positiva e curvaturas principais imaginárias}

Suponha que $X$ é uma superfície não Weingarten-diagonalizável com discriminante do polinômio característico $\triangle<0$. Neste caso o operador Weingarten é diagonalizável sobre o corpo dos números complexos e assim temos o termo curvaturas principais imaginárias. Este caso foi tratado em 2002 no artigo On time-like surfaces of positive constant gaussian curvature and imaginary principals curvatures [12, no qual C.H. Gu, H.S. Hu e J. Inoguchi mostraram que apesar das curvaturas principais serem imaginárias, a superfície ainda admite linhas assintóticas reais, e que é possível condicionar a existência de superfícies com estas propriedades a existência de soluções da equação de cosh-gordon $\alpha_{u v}=\cosh \alpha$.

Seguindo então [12] vamos mostrar como se dá tal relação. 
Seja $\left\{e_{1}, e_{2}, e_{3}\right\}$ um Referencial Móvel Nulo adaptado a $\mathbb{X}$ como na Definição 2.18 , com parâmetro $\mu=\frac{1}{2} e^{\alpha}$.

Suponhamos que $\mathbb{X}(u, v)$ esteja parametrizada por algum sistema de coordenadas especial $(u, v)$, na qual o co-referencial móvel e as equações de estrutura, definidas pelas Equações 2.26) e 2.27), admitam a seguinte decomposição na base $\{d u, d v\}$,

$$
\begin{aligned}
\omega^{1} & =d u-e^{-\alpha} d v, \\
\omega^{2} & =-e^{-\alpha} d u-d v, \\
\omega_{3}^{1} & =-d u-e^{-\alpha} d v, \\
\omega_{3}^{2} & =-e^{-\alpha} d u+d v .
\end{aligned}
$$

Chamaremos tais coordenadas $(u, v)$ de Coordenadas Nulas de Gu-Hu-Inoguchi e o respectivo Referencial Móvel será chamado de Referencial Móvel Nulo de Gu-Hu-

\section{Inoguchi.}

Nestas condições, a Equação de Estrutura 2.34 nos garante que

$$
\begin{aligned}
& \omega_{1}^{3}=\frac{1}{2} d u-\frac{1}{2} e^{\alpha} d v \\
& \omega_{2}^{3}=\frac{1}{2} e^{\alpha} d u+\frac{1}{2} d v .
\end{aligned}
$$

As Equações de Estrutura 2.35 e 2.36), nos permite calcular $\omega_{1}^{1}$ e $\omega_{2}^{2}$, que serão respectivamente dadas por

$$
\begin{aligned}
& \omega_{1}^{1}=\alpha_{u} d u \\
& \omega_{2}^{2}=\alpha_{v} d v .
\end{aligned}
$$

Escrevendo a primeira e segunda forma $I$ e $I I$ de $\mathbb{X}$ conforme as Equações 2.44) e 2.45), temos

$$
\begin{aligned}
I & =-d u \otimes d u-2 \sinh \alpha d u \otimes d v+d v \otimes d v \\
I I & =-2 \cosh \alpha d u \otimes d v .
\end{aligned}
$$

Assim identificamos os coeficientes da primeira e segunda formas fundamentais por,

$$
\begin{gathered}
E=1, \quad F=-\sinh \alpha, \quad G=1, \\
e=0, \quad f=-\cosh \alpha, \quad \text { e } \quad g=0 .
\end{gathered}
$$


Verificamos que se $\mathbb{X}(u, v)$, admite tal parametrização, então de acordo com as Equações 1.13, 1.14 e 1.22, temos que,

$$
\begin{array}{r}
K=\epsilon_{3} \frac{e g-f^{2}}{E G-F^{2}}=1, \\
H=\frac{\epsilon_{3}}{2} \frac{e G-2 f F+g E}{E G-F^{2}}=\tanh \alpha, \\
\triangle=4\left(H^{2}-\epsilon_{3} K\right)=\tanh \alpha<0 .
\end{array}
$$

De modo que a Superfície $\mathbb{X}(u, v)$ tem curvatura Gaussiana constante e curvaturas principais imaginárias. Além disso, podemos verificar que as Equações de Codazzi 2.40) e 2.41), dadas por,

$$
\begin{aligned}
& d \omega_{3}^{1}=\omega_{3}^{1} \wedge \omega_{1}^{1}, \\
& d \omega_{3}^{2}=\omega_{3}^{2} \wedge \omega_{2}^{2},
\end{aligned}
$$

são automaticamente satisfeitas, e a Equação de Gauss (2.38), é equivalente a equação de cosh-Gordon,

$$
\alpha_{u v}=\cosh \alpha .
$$

Com isto, mostramos que a existência do Referencial Móvel e do Co-Referencial Móvel nulo de $\mathrm{Gu}-\mathrm{Hu}$-Inoguchi é equivalente a dizer que a superfície $\mathbb{X}(u, v)$ tem curvatura Gaussiana constante $K=1$ e curvaturas principais imaginárias. Além disso a existência de tal Superfície é equivalente a função $\alpha$ satisfazer a Equação de Gauss. Deste modo obtemos o seguinte resultado.

Teorema 3.9. Existe uma relação biunívoca entre as soluções da equação de cosh-Gordon com a existência, a menos de um movimento rígido no espaço Minkowski, de superfícies tipo-tempo com curvatura Gaussiana constante positiva com uma duas direções principais imaginárias.

\subsection{Classificação das superfícies de curvatura constante não nula}

A tabela a seguir resume os resultados obtidos nesta seção. A primeira coluna refere-se à superfície parametrizada em questão. A segunda coluna indica se a curvatura Gaussiana é uma constante positiva ou negativa. A terceira coluna indica qual o valor do discrimininate do polinômio característico da superfície, conforme a equação 1.21). A quarta coluna indica 
qual as coordenadas são utilizadas para obter a EDP natural respectiva, que se encontra na quinta coluna. As oito linhas representam todos os possíveis casos de superfícies regulares não-degeneradas com curvaturas constantes não nulas.

Nesta tabela também abreviamos o produto tensorial, pela justaposição, isto é, $w \otimes \bar{\omega}:=$ $\omega \bar{\omega}$, também $(\omega)^{2}$ denota $\omega \omega$. Como tais produtos tensoriais são usados para denotar formas quadráticas, fica bem definido escrever $\omega \bar{\omega}=\bar{\omega} \omega$. 


\begin{tabular}{|c|c|c|c|c|c|c|c|c|c|c|c|c|c|c|c|c|c|c|}
\hline 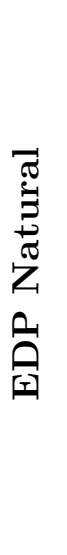 & & 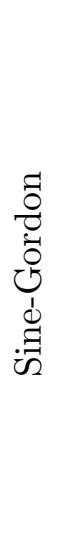 & 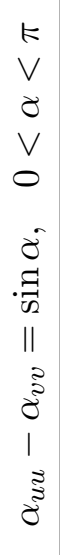 & 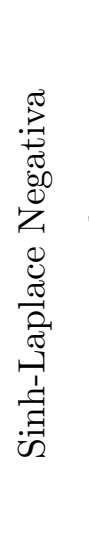 & 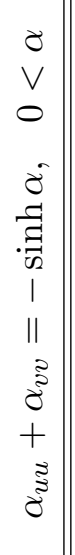 & 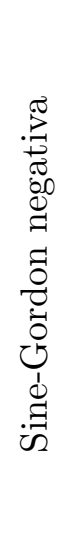 & 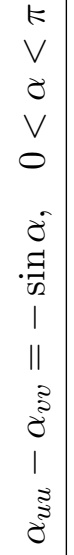 & 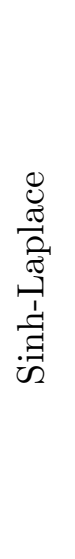 & 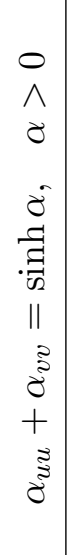 & 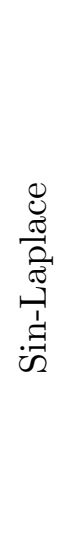 & 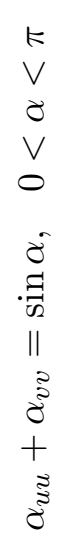 & 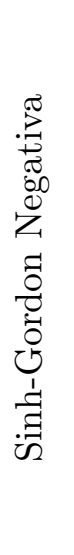 & 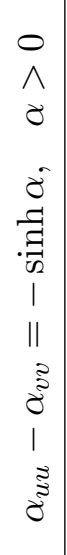 & 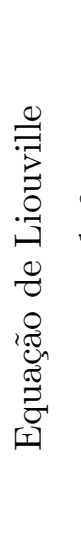 & 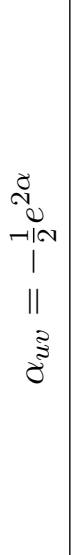 & $\begin{array}{l}\tilde{0} \\
0 \\
0 \\
0 \\
0 \\
\frac{1}{1} \\
\frac{1}{01} \\
0 \\
0\end{array}$ & $\begin{array}{c}0 \\
0 \\
07 \\
0 \\
0 \\
\| 1 \\
0 \\
0 \\
0\end{array}$ & \\
\hline 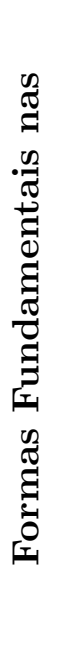 & 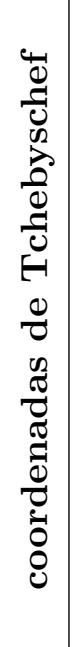 & 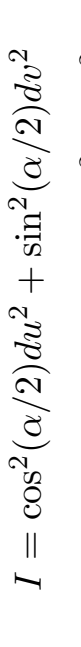 & 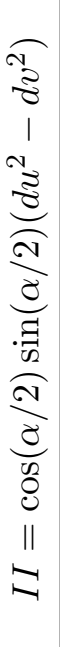 & 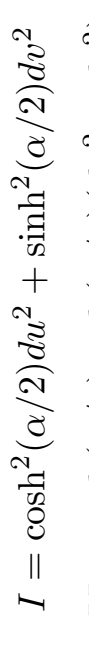 & 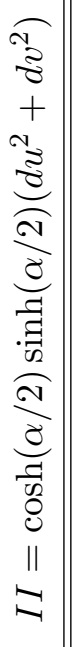 & 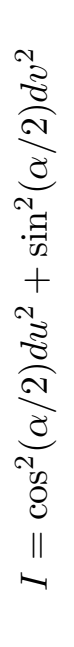 & 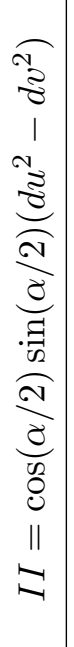 & 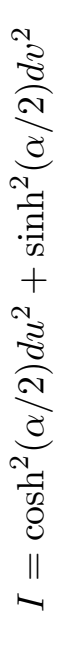 & 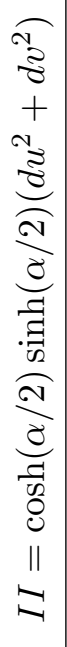 & 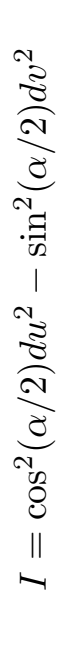 & 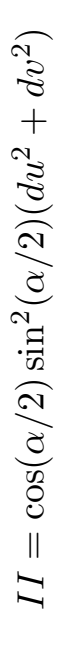 & 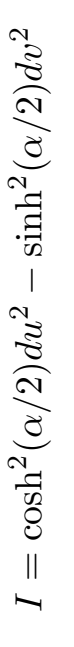 & 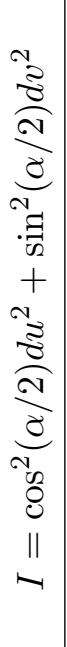 & 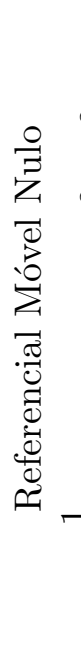 & 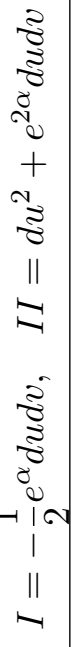 & 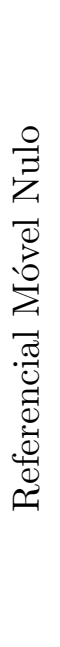 & 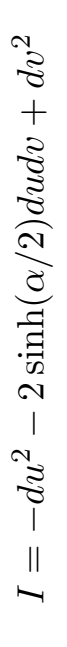 & 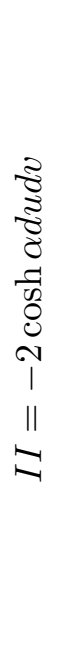 \\
\hline 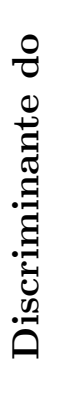 & 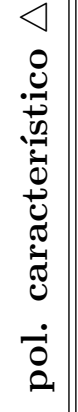 & 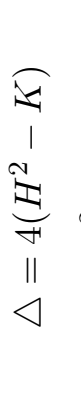 & 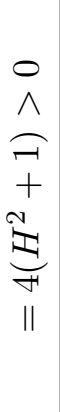 & 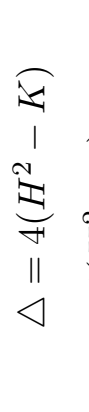 & 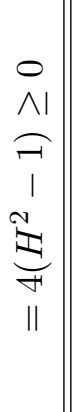 & 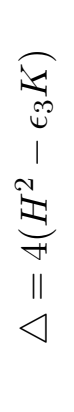 & 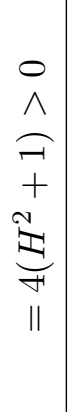 & 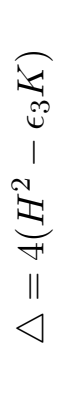 & 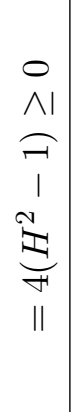 & 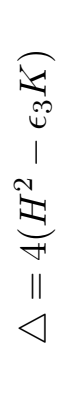 & 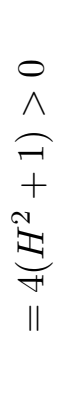 & 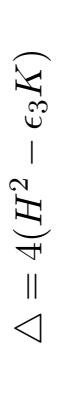 & 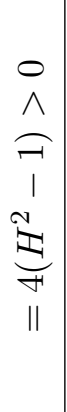 & 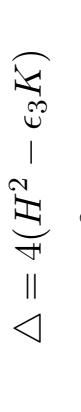 & 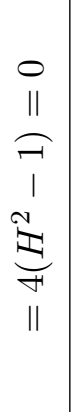 & 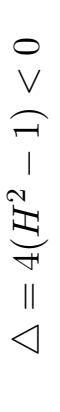 & 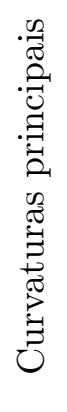 & 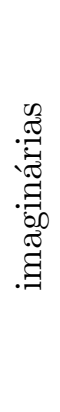 \\
\hline 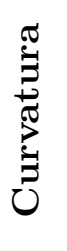 & 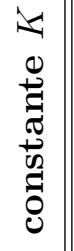 & \begin{tabular}{l}
$r$ \\
11 \\
\multirow{2}{*}{}
\end{tabular} & & $\begin{array}{l}\overrightarrow{+} \\
\| \\
\vec{z}\end{array}$ & & $\begin{array}{l}+ \\
\| \\
* 1\end{array}$ & & 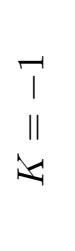 & & $\begin{array}{l}1 \\
11 \\
11\end{array}$ & & $\begin{array}{l}\overrightarrow{+} \\
\| \\
\approx\end{array}$ & & $\begin{array}{l}+ \\
\| \\
* 1\end{array}$ & & $\begin{array}{l}\overrightarrow{+} \\
\| \\
\approx\end{array}$ & & \\
\hline 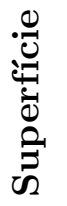 & & 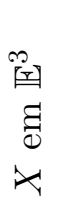 & & 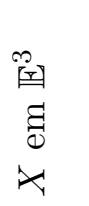 & & $\begin{array}{l}\stackrel{\infty}{\ominus} \\
\overrightarrow{0} \\
\ddot{A}\end{array}$ & 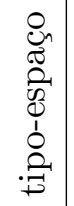 & & & $\begin{array}{l}\stackrel{\infty}{\ominus} \\
\overrightarrow{0} \\
\ddot{A}\end{array}$ & 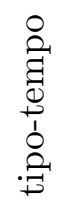 & & & & & & & \\
\hline
\end{tabular}




\section{Capítulo 4}

\section{Transformações de Bäcklund em $\mathbb{L}^{3}$}

Neste capítulo trataremos da transformação de Bäcklund e suas consequências.

Historicamente, a transformação de Bäcklund introduzida pelo físico-matemático sueco Albert Victor Bäcklund (1845-1922). Esta é uma transformação geométrica entre superfícies, no Espaço Euclidiano $\mathbb{E}^{3}$, com curvatura constante negativa na qual permite construir novas superfícies de curvatura constante negativa à partir de uma dada superfície de curvatura constante negativa. Esta transformação de Bäcklund ganhou destaque na literatura pela sua aplicação na teoria de sólitons e na teoria de sistemas integráveis. Uma vez que as superfícies de curvatura constante negativa estão relacionadas com soluções da equação diferencial parcial de sine-Gordon $\left(u_{x x}-u_{y y}=\sin u\right)$ esta transformação, a princípio geométrica, ganha um caráter analítico pois, permite transformar uma solução desta equação em outra solução. Deste modo esta transformação apresentou um método útil tanto para o campo da geometria diferencial quanto para o campo das EDP's, no sentido que a cada nova solução para equação de sine-Gordon é possível obter um exemplo, não trivial, de superfície com curvatura constante negativa, e reciprocamente, a cada superfície de curvatura constante negativa é possível construir uma solução a equação de sine-Gordon. Deste modo temos duas facetas para as transformações de Bäcklund; uma geométrica e outra analítica.

As transformações de Bäcklund para superfícies em $\mathbb{E}^{3}$, atualmente, compõem um tema clássico e bem difundido na literatura. Paralelamente, quando consideramos a Geometria não-euclidiana no espaço-tempo de Minkowski, temos as seguintes questões naturais:

1. Sendo a transformação de Bäcklund uma transformação geométrica, temos um análogo para superfícies no espaço de Minkowski?

2. Tais transformações, se bem definidas, permitem construção de novas superfícies com 
curvatura constante no espaço de Minkowski?

3. Tais transformações geométricas, se bem definidas, possuem também o mesmo caráter analítico que as transformações de superfícies no espaço Euclidiano?

Este capítulo será dedicado a responder tais questões naturais. Veremos que a resposta para primeira pergunta é afirmativa. É possível introduzir transformações de Bäcklund para superfícies no espaço de Minkowski. A segunda pergunta também terá uma resposta afirmativa. A transformação de Bäcklund ocorrerá entre superfícies de curvatura constante, na qual dependendo do caráter causal da superfície a transformação de Bäcklund ocorrerá para superfícies com curvatura constante negativa ou positiva. A resposta para terceira pergunta se afigurará afirmativa. Ainda mais, a transformação analítica correspondente trará soluções, não somente para a equação de sine-Gordon, mas também, dependendo do caráter causar da superfície, para as equações de sine-Gordon negativa $\left(u_{x x}-u_{y y}=-\sin u\right)$, sinh-Laplace $(\Delta u=\sinh u)$, sin-Laplace $(\Delta u=\sin u)$, sinh-Gordon negativa $\left(u_{x x}-u_{y y}=-\sinh u\right)$, coshGordon $\left(u_{x x}-u_{y y}=\cosh u\right)$ e também para a equação de Liouville $\left(u_{x y}=\frac{1}{2} \mathrm{e}^{u}\right)$.

Introduziremos a transformação de Bäcklund para superfícies em $\mathbb{L}^{3}$, assim como é feita no caso Euclidiano, isto é, a partir da congruência pseudo-esférica. Todas as definições e resultados serão enunciados para o caso Euclidiano e adaptados, de forma analoga, para para o espaço de Minkowski. Assim será imediata a comparação entre os casos Euclidiano e não-Euclidiano.

Começaremos formalizando o conceito de Congruência Pseudo-Esférica e então construiremos a transformação de Bäcklund. Finalizaremos este capítulo com algumas consequências das transformações de Bäcklund, como sua relação com as EDP's e seu caráter analítico.

\subsection{Congruência Pseudo-Esférica no espaço Euclidiano}

Esta seção terá como referência [20] e [1].

No que segue denotemos por $\mathbb{X}: U \subset \mathbb{R}^{2} \rightarrow \mathbb{E}^{3}$ uma superfície parametrizada regular simples cujo traço é $S=X(U) \subset \mathbb{R}^{3}$.

Definição 4.1. Congruência pseudo-esférica em $\mathbb{E}^{3}$ Seja $\mathcal{B}: S \rightarrow \widetilde{S}$ um difeomorfismo entre duas superfícies parametrizadas regulares simples. Dizemos que $\mathcal{B}$ é uma congruência pseudo-esférica desde que 
1. O vetor $\vec{r}(p)=\overline{p \mathcal{B}(p)}$ pertença simultaneamente ao plano tangente de $X$ e $\widetilde{X}$ em $p$ e $\mathcal{B}(p)$, respectivamente.

2. O módulo do vetor é uma constante positiva, isto é, $|\vec{r}(p)|=\lambda>0$ para todo $p \in S$.

3. $O$ ângulo, $\phi$, entre as normais $N$ e $\widetilde{N}$ da superfícies $S$ e $\widetilde{S}$ em cada ponto $p$ e $\mathcal{B}(p)$, respectivamente, é uma constante não nula.

Notação 4.1. Em uma congruência pseudo-esférica denotamos as constantes, $\lambda=|\vec{r}|>0$ e $\phi \in(0, \pi)$ por distância de congruência e ângulo de congruência, respectivamente.

Apesar dos três itens da definição aparentarem ser condições bastante restritivas, congruências pseudo-esféricas entre superfícies existem. Veja o Exemplo 4.1 abaixo. Ainda é possível mostrar, conforme veremos, que a congruência pseudo-esférica ocorre somente entre superfícies que têm curvatura constante negativa (conforme o Teorema de Bäcklund (4.1)), daí a motivação para o termo pseudo-esférica.

Exemplo 4.1. Sejam $X$ a pseudo-esfera, dada pela superfície parametrizada

$$
X(u, v)=(\sinh u \cos v, \sinh u \operatorname{sen} v, u-\tanh u) ; \quad u>0, \quad 0<v<2 \pi
$$

e $\widetilde{X}$ uma superfície, com parâmetros $(u, v)$, dada por

$$
\widetilde{X}=X+\cos \theta \operatorname{coth} u X_{u}+\operatorname{sen} \theta \cosh u X_{v}
$$

em que $\theta(u, v)$ é uma função definida implicitamente pela relação $\operatorname{coth} \frac{\theta}{2}=-v \operatorname{sech} u$. Então a transformação $\mathcal{B}$ que associa a cada $X(u, v)$ o ponto $\widetilde{X}(u, v)$ é uma congruência pseudoesférica com parâmetros $\lambda=1$ e $\phi=\pi / 2$.

O teorema a seguir nos garante que uma condição necessária para existência de congruência pseudo-esférica entre duas superfícies, é que as duas superfícies devem ter a mesma curvatura constante negativa.

Teorema 4.1. (Teorema de Bäcklund em $\mathbb{E}^{3}$ ) Sejam $S, \widetilde{S} \subset \mathbb{R}^{3}$ duas superfícies parametrizadas regulares simples no espaço Euclidiano $\mathbb{E}^{3}$. Então, se existe uma congruência pseudo-esférica $\mathcal{B}: S \rightarrow \widetilde{S}$ entre $S$ e $\widetilde{S}$ com distância $\lambda$ e ângulo $\phi$, as duas superfícies deverão ter a mesma curvatura constante negativa igual a $-\frac{\operatorname{sen}^{2} \phi}{\lambda^{2}}$. 
Demonstração: A idéia da demonstração é construir um referencial móvel para as superfícies $S$ e $\widetilde{S}$, em função de $\lambda$ e $\phi$ e então usar a Equação de Gauss para relacionar a curvatura Gaussiana com os parâmetros $\lambda$ e $\phi$.

Suponhamos que exista $\mathcal{B}: S \rightarrow \widetilde{S}$ uma congruência pseudo-esférica entre as superfícies $S=X(U)$ e $\widetilde{S}=\widetilde{X}(U)$ com normal $N$ e $\widetilde{N}$ e curvatura Gaussiana $K$ e $\widetilde{K}$ respectivamente. Partindo de um referencial móvel adaptado a superfície $S,\left\{e_{1}, e_{2}, e_{3}\right\}$, tal que $e_{1}$ esteja na direção de $\vec{r}$, vamos tomar um referêncial móvel adaptado a $\widetilde{S}$. Primeiramente a condição 1) da Definição (4.1) nos permite tomar $\widetilde{e}_{1}=e_{1}$ já que este vetor pertence ao espaço tangente das duas superfícies. Em seguida, vamos expressar $\widetilde{e}_{3}=\widetilde{N}$ em termos da base $\left\{e_{i}\right\}$, isto é, vamos determinar os coeficientes $\mu_{i}$ tais que,

$$
\widetilde{e}_{3}=\mu_{1} e_{1}+\mu_{2} e_{2}+\mu_{3} e_{3} .
$$

A condição 3) da Definição 4.1) nos garante que,

$$
\left\langle e_{3}, \widetilde{e}_{3}\right\rangle=\cos \phi, \quad \text { ou seja, } \quad \mu_{3}=\cos \phi
$$

da condição 1) da Definição 4.1) temos,

$$
\left\langle e_{1}, \widetilde{e}_{3}\right\rangle=0, \quad \text { temos que } \quad \mu_{1}=0
$$

e por último pedindo que

$$
\left\langle\widetilde{e}_{3}, \widetilde{e}_{3}\right\rangle=1, \quad \log 0 \quad \mu_{2}^{2}+\cos ^{2} \phi=1
$$

Assim $\widetilde{e}_{3}=\mu_{2} e_{2}+\cos \phi e_{3}, \operatorname{com} \mu_{2}= \pm \operatorname{sen} \phi$. Tomando $\widetilde{e}_{2}=\widetilde{e}_{3} \times_{\mathbb{E}^{3}} \widetilde{e}_{1}$ temos

$$
\widetilde{e}_{2}=\widetilde{e}_{3} \times_{\mathbb{E}^{3}} \widetilde{e}_{1}=\left|\begin{array}{rrr}
e_{1} & e_{2} & e_{3} \\
0 & \mu_{2} & \cos \phi \\
1 & 0 & 0
\end{array}\right|=\cos \phi e_{2}-\mu_{2} e_{3} .
$$

Esta relação entre os referenciais móveis adaptados a $S$ e $\widetilde{S}$ nos permitirá relacionar os respectivos co-referenciais móveis. Denote por $\omega^{i}$ e $\omega_{i}^{j}, i, j \in\{1,2,3\}$, o co-referencial móvel e as formas de conexão, respectivamente, associados ao referencial móvel $\left\{e_{i}\right\}$ e $\widetilde{\omega}^{i}$ e $\widetilde{\omega}_{i}^{j}$ o co-referencial móvel e as formas de conexão, respectivamente, associados ao referencial móvel $\left\{\widetilde{e}_{i}\right\}$.

Da condição 2) da Definição 4.1 podemos escrever

$$
\widetilde{X}=X+\lambda e_{1}
$$


De onde segue que

$$
d \tilde{X}=d X+\lambda d e_{1}
$$

Uma vez que $d X=\omega^{1} e_{1}+\omega^{2} e_{2}, d e_{1}=\omega_{1}^{2} e_{2}+\omega_{1}^{3} e_{3}$ e

$$
d \widetilde{X}=\widetilde{\omega}^{1} \widetilde{e}_{1}+\widetilde{\omega}^{2} \widetilde{e}_{2}=\widetilde{\omega}^{1} e_{1}+\widetilde{\omega}^{2}\left(\cos \phi e_{2}-\mu_{2} e_{3}\right)
$$

temos que a Equação 4.1 é equivalente a

$$
\widetilde{\omega}^{1} e_{1}+\widetilde{\omega}^{2} \cos \phi e_{2}-\widetilde{\omega}^{2} \mu_{2} e_{3}=\omega^{1} e_{1}+\omega^{2} e_{2}+\lambda\left(\omega_{1}^{2} e_{2}+\omega_{1}^{3} e_{3}\right) .
$$

Usando a independência linear do referencial móvel $\left\{e_{i}\right\}$ verificamos as seguintes relações;

$$
\begin{array}{r}
\widetilde{\omega}^{1}=\omega^{1} \\
\widetilde{\omega}^{2} \cos \phi=\omega^{2}+\lambda \omega_{1}^{2} \\
\widetilde{\omega}^{2}\left(-\mu_{2}\right)=\lambda \omega_{1}^{3},
\end{array}
$$

em que $\mu_{2}= \pm \operatorname{sen} \phi$. E pela condição 3) da Definição (4.1) temos que $\phi \in(0, \pi)$ e assim destas duas últimas relações, temos que

$$
\omega_{1}^{2}=-\frac{1}{\lambda} \omega^{2}-\frac{\cos \phi}{\mu_{2}} \omega_{1}^{3}
$$

Tomando a diferencial exterior desta expressão segue que;

$$
d \omega_{1}^{2}=-\frac{1}{\lambda} d \omega^{2}-\frac{\cos \phi}{\mu_{2}} d \omega_{1}^{3}
$$

Usando as Equações de Estrutura (2.4) e 2.7) temos

$$
\begin{aligned}
d \omega_{1}^{2} & =-\frac{1}{\lambda} \omega^{1} \wedge \omega_{1}^{2}-\frac{\cos \phi}{\mu_{2}}\left(\omega_{1}^{2} \wedge \omega_{2}^{3}\right) \\
& =\omega_{1}^{2} \wedge\left(\frac{1}{\lambda} \omega^{1}-\frac{\cos \phi}{\mu_{2}} \omega_{2}^{3}\right) .
\end{aligned}
$$

Substituindo $\omega_{1}^{2}$ pela Equação 4.2, segue que

$$
\begin{aligned}
d \omega_{1}^{2} & =\left(-\frac{1}{\lambda} \omega^{2}-\frac{\cos \phi}{\mu_{2}} \omega_{1}^{3}\right) \wedge\left(\frac{1}{\lambda} \omega^{1}-\frac{\cos \phi}{\mu_{2}} \omega_{2}^{3}\right) \\
& =\frac{1}{\lambda^{2}} \omega^{1} \wedge \omega^{2}-\frac{\cos \phi}{\lambda \mu_{2}}\left(\omega_{1}^{3} \wedge \omega^{1}-\omega^{2} \wedge \omega_{2}^{3}\right)+\frac{\cos ^{2} \phi}{\mu_{2}^{2}} \omega_{1}^{3} \wedge \omega_{2}^{3} \\
& =\frac{1}{\lambda^{2}} \omega^{1} \wedge \omega^{2}+\frac{\cos ^{2} \phi}{\mu_{2}^{2}} \omega_{1}^{3} \wedge \omega_{2}^{3},
\end{aligned}
$$

em que na última passagem usamos a Equação de Estrutura 2.5. 
Partindo da Equação de Estrutura (2.6) juntamente com a Equação de Gauss (2.13), $d \omega_{1}^{2}=-K \omega^{1} \wedge \omega^{2}=\omega_{1}^{3} \wedge \omega_{3}^{2}$, temos que a Equação 4.3. é equivalente a

$$
-K \omega^{1} \wedge \omega^{2}=\frac{1}{\lambda^{2}} \omega^{1} \wedge \omega^{2}+\frac{\cos ^{2} \phi}{\mu_{2}^{2}}\left(K \omega^{1} \wedge \omega^{2}\right) .
$$

Daí decorre que,

$$
\omega^{1} \wedge \omega^{2}\left(\frac{1}{\lambda^{2}}+\frac{\cos ^{2} \phi}{\mu_{2}^{2}} K+K\right)=0 .
$$

Como as 1-formas $\omega^{1}$ e $\omega^{2}$ são linearmente independentes, garantimos que o termo entre parênteses é nulo em todos os pontos da superfície. Logo temos que

$$
K=-\frac{\mu_{2}^{2}}{\left(\mu_{2}^{2}+\cos \phi\right) \lambda^{2}} .
$$

Como $\mu_{2}= \pm \operatorname{sen} \phi$ temos que a curvatura Gaussiana de $S$ é constante negativa dada por

$$
K=-\frac{\operatorname{sen}^{2} \phi}{\lambda^{2}}
$$

Finalmente pela simetria do argumento, a superfície $\widetilde{S}$ terá a mesma curvatura Gaussiana constante negativa;

$$
\widetilde{K}=-\frac{\operatorname{sen}^{2} \phi}{\lambda^{2}}
$$

Assim o teorema está demonstrado.

\subsection{Congruência Pseudo-Esférica Generalizada}

Considere $\mathbb{X}: U \subset \mathbb{R}^{2} \rightarrow \mathbb{L}^{3}$ e $\widetilde{\mathbb{X}}: \widetilde{U} \subset \mathbb{R}^{2} \rightarrow \mathbb{L}^{3}$ duas superfícies parametrizadas regulares simples em $\mathbb{L}^{3}$. Denotaremos por $M=\mathbb{X}(U) \subset \mathbb{L}^{3}$ e $\widetilde{M}=\widetilde{\mathbb{X}}(\widetilde{U}) \subset \mathbb{L}^{3}$ o traço das respectivas superfícies, $p \in M$ e $\widetilde{p} \in \widetilde{M}$ pontos pertencentes aos respectivos traços das superfícies.

Definição 4.2. (Congruência pseudo-esférica em $\mathbb{L}^{3}$ ). Seja $\mathcal{B}: M \rightarrow \widetilde{M}$ um difeomorfismo entre duas superfícies parametrizadas simples em $\mathbb{L}^{3}$. Denote $\mathcal{B}(p)=\widetilde{p} \in \widetilde{M}$ com $p \in M$. Dizemos que $\mathcal{B}$ é uma Congruência Pseudo-Esférica desde que, sejam satisfeitas as três condições abaixo:

1. o vetor $\vec{r}(p)=\overline{p \widetilde{p}}$, pertence ao espaço tangente de $M$ e $\widetilde{M}$ para cada $p \in M$;

2. o comprimento do seguimento $\overline{p \widetilde{p}}$ é uma constante positiva para cada $p \in M$, isto é, a norma $|\vec{r}(p)|>0$ é constante; 
3. o produto $\langle N(p), \widetilde{N}(\widetilde{p})\rangle=k$ é constante, em que $N$ e $\widetilde{N}$, não colineares, são as normais as superfícies $M$ e $\widetilde{M}$ respectivamente, para cada $p \in M$.

Além disso, se $\mathcal{B}$ é uma congruência pseudo-esférica entre $M$ e $\widetilde{M}$, dizemos que a congruência pseudo-esférica é tipo-espaço (tipo-tempo) desde que, o vetor $\vec{r}(p)=\overline{p \widetilde{p}}$ seja tipo-espaço (tipotempo) para todo $p \in M$.

No que segue, estaremos interessados nos seguintes casos.

\section{Caso A}

$\mathcal{B}$ é uma congruência pseudo-esférica tipo-espaço entre superfícies $M$ e $\widetilde{M}$ tipo-espaço.

Neste caso $N$ e $\widetilde{N}$ são vetores tipo-tempo, de modo que fica definido um ângulo hiperbólico entre os vetores normais se estes pertencem ao mesmo cone tipo-tempo. Em geral existirá um único número real $\phi \in(0,+\infty)$ satisfazendo,

$$
\langle N(p), \widetilde{N}(\widetilde{p})\rangle=\tau \cosh (\phi),
$$

em que, $\widetilde{p}=\mathcal{B}(p)$ e $\tau=-1$, se $\widetilde{N} \in \mathcal{C}(N)$ ou $\tau=+1$, se $\widetilde{N} \in \mathcal{C}(-N)$.

\section{Caso B}

$\mathcal{B}$ é uma congruência pseudo-esférica tipo-espaço entre superfícies $M$ e $\widetilde{M}$ tipo-tempo.

Neste caso $N$ e $\widetilde{N}$ são vetores tipo-espaço e $\vec{r}$ é um vetor tipo-espaço. Denotando $\vec{r}^{\perp}=$ $\vec{r} \times N$, temos que $\vec{r}^{\perp}$ é um vetor tipo-tempo. De fato, $\left\{\vec{r}, N, \vec{r}^{\perp}\right\}$ formam uma base ortonormal para $\mathbb{L}^{3}$ de modo que $\vec{r}, N$ serem vetores tipo-espaço implica em $\vec{r}^{\perp} \in \mathcal{T}$. Ainda temos que $\widetilde{N} \in\left\langle\left\{N, \vec{r}^{\perp}\right\}\right\rangle$ pertence a um plano Lorentziano de modo que existe um único ângulo hiperbólico, $\phi \in(0,+\infty)$, entre os vetores tipo-espaço tal que

$$
\langle N, \widetilde{N}\rangle=\tau \cosh \phi
$$

no qual $\tau=-1$ se, $N$ e $\widetilde{N}$ pertencem a mesma componente conexa de $\mathbb{U}^{2}$, ou $\tau=+1$ caso contrário.

\section{Caso C}

$\mathcal{B}$ é uma congruência pseudo-esférica tipo-tempo entre superfícies $M$ e $\widetilde{M}$ tipo-tempo.

Neste caso, teremos que $N, \tilde{N} \in\langle\{\vec{r}\}\rangle$ são vetores tipo-espaço em um plano Riemanniano. Assim existe um ângulo $\phi \in(0, \pi)$ tal que

$$
\langle N, \tilde{N}\rangle=\cos (\phi)
$$




\section{Caso D}

$\mathcal{B}$ é uma congruência pseudo-esférica tipo-espaço entre superfícies $M$ tipo-espaço e $\widetilde{M}$ tipo-tempo.

Neste caso, $N \in \mathcal{T}$ e $\widetilde{N} \in \mathcal{E}$ de modo que não fica definido um ângulo entre estes dois vetores. Entretanto, existe um único número real $\phi \in[0,+\infty)$ tal que

$$
\langle N, \tilde{N}\rangle=\tau \sinh \phi
$$

na qual $\tau=\operatorname{sinal}(\langle N, \tilde{N}\rangle)$.

Notação 4.2. Em todos os casos A, B, C e D denotamos o número $\phi$ por ângulo da congruência pseudo-esférica, que será de fato um ângulo para os casos A-C. Denotaremos $\lambda=|\vec{r}|$ o módulo do vetor $\vec{r}$ e diremos que $\lambda$ é a distância da congruência pseudo-esférica.

Com esta notação, temos que se $\mathcal{B}$ é uma congruência-pseudo esférica, as condições 2) e 3) da Definição (4.2) nos asseguram que os parâmetros da congruência $\lambda$ e $\phi$ são constantes.

Notemos que se $\mathcal{B}$ é uma congruência pseudo-esférica entre duas superfícies não-degeneradas sempre podemos supor que, localmente, a congruência se encontra em um dos casos A-D. De fato, se as superfícies são não-degeneradas então elas não são do tipo-luz, de modo que localmente as superfícies preservam o caráter causal e também por continuidade da aplicação $\mathcal{B}$ garantimos que o caráter causal da congruência é, localmente, mantido. Note também que não é possível existir uma congruência pseudo-esférica tipo-tempo entre superfícies tipoespaço ou mesmo existir uma congruência tipo-tempo entre duas superfícies tipo-espaço e tipo-tempo respectivamente, pois nos dois casos teríamos que o vetor de congruência $\vec{r}$ seria um vetor tipo-tempo contido em um plano tangente Riemanniano, o que seria um absurdo.

Observação 4.1. Se a aplicação $\mathcal{B}: M \rightarrow \widetilde{M}$ é uma congruência pseudo-esférica, temos que o conjunto de retas tangentes, nas direções de $\vec{r}(p)$, formam um tipo de congruência de retas dita Congruência pseudo-esférica de retas em $\mathbb{L}^{3}$.

O seguinte resultado é uma condição necessária para a ocorrência de congruência pseudoesféricas. Elas nos garante que a congruência pseudo-esférica só é possível para superfícies que possuem a mesma curvatura Gaussiana constante. Este resultado é uma adaptação dos resultados de [19] Palmer (1990): Teorema I página 2872, [11] C. H. Gu at al (2005): Teorema 4.8 página 150 e também [15] L. V. McNertney (1980): Teorema 4.2 página 86. 
Teorema 4.2. Sejam $\mathbb{X} e \widetilde{\mathbb{X}}$ duas superfícies parametrizadas regulares simples não-degeneradas em $\mathbb{L}^{3}$. Se existe uma congruência pseudo-esférica $\mathcal{B}: M \rightarrow \widetilde{M}$, então devemos ter que curvatura Gaussiana $K$ e $\widetilde{K}$ de $\mathbb{X}$ e $\widetilde{\mathbb{X}}$ são iguais e constantes.

Demonstração: Suponhamos que exista $\mathcal{B}: M \rightarrow \widetilde{M}$ uma congruência pseudo-esférica com distância $\lambda>0$ e ângulo $\phi \geqslant 0$ entre as superfícies $\mathbb{X}$ e $\widetilde{\mathbb{X}}$. A idéia da demonstração é partir de um referencial móvel para superfície $\mathbb{X}$ construir um referencial móvel para a superfície $\widetilde{\mathbb{X}}$ de modo que os co-referenciais móveis sejam escritos em termos dos parâmetros $\lambda$ e $\phi$ para então usar a Equação de Gauss e obter a curvatura Gaussiana em função destes parâmetros, de modo que esta relação expressará $K$ e $\widetilde{K}$ em termos das constantes $\lambda$ e $\phi$, e daí concluiremos a tese do teorema.

Comecemos com $\left\{e_{1}, e_{2}, e_{3}\right\}$ um referencial móvel adaptado a superfície $\mathbb{X}$. Pela condição 1) da Definição 4.2 podemos tomar $e_{1}=\frac{\vec{r}}{\lambda}$ na direção de congruência. Vamos construir $\left\{\widetilde{e}_{1}, \widetilde{e}_{2}, \widetilde{e}_{3}\right\}$ um referencial móvel adaptado a superfície $\widetilde{\mathbb{X}}$. Novamente pela condição 1) da Definição 4.2 podemos tomar $\widetilde{e}_{1}=e_{1}$ na direção de congruência. Vamos escrever $\widetilde{e}_{3}=\widetilde{N}$ na base $\left\{e_{i}\right\}$. Para isso vamos determinar os coeficientes $\mu_{i}$ tais que

$$
\widetilde{e}_{3}=\mu_{1} e_{1}+\mu_{2} e_{2}+\mu_{3} e_{3}
$$

Primeiramente, $0=\left\langle\widetilde{e}_{3}, \widetilde{e}_{1}\right\rangle=\left\langle\widetilde{e}_{3}, e_{1}\right\rangle$, assim $\epsilon_{1} \mu_{1}=0$, em que $\epsilon_{i}=\left\langle e_{i}, e_{i}\right\rangle$, e consequentemente

$$
\mu_{1}=0
$$

A condição 3) da Definição (4.2) nos assegura que, $\left\langle e_{3}, \widetilde{e}_{3}\right\rangle=k$, assim garantimos que

$$
\mu_{3}=\epsilon_{3} k
$$

Por último, denotando $\widetilde{\epsilon}_{i}=\left\langle\widetilde{e}_{i}, \widetilde{e}_{i}\right\rangle$, temos que

$$
\widetilde{\epsilon}_{3}=\left\langle\widetilde{e}_{3}, \widetilde{e}_{3}\right\rangle=\epsilon_{2} \mu_{2}^{2}+\epsilon_{3} \mu_{3}^{2}=\epsilon_{2} \mu_{2}^{2}+\epsilon_{3} k^{2}
$$

Logo

$$
\mu_{2}=\sigma \sqrt{\epsilon_{2} \widetilde{\epsilon}_{3}-\epsilon_{2} \epsilon_{3} k^{2}} \quad \sigma \in\{+1,-1\}
$$

Podemos determinar $\widetilde{e}_{2}$ pelo produto Lorentziano de $\widetilde{e}_{1} \operatorname{com} \widetilde{e}_{3}$,

$$
\widetilde{e}_{2}=\widetilde{e}_{1} \times \widetilde{e}_{3}=\left|\begin{array}{rrr}
\epsilon_{1} e_{1} & \epsilon_{2} e_{2} & \epsilon_{3} e_{3} \\
1 & 0 & 0 \\
\mu_{1} & \mu_{2} & \mu_{3}
\end{array}\right|=-\mu_{3} \epsilon_{2} e_{2}+\mu_{2} \epsilon_{3} e_{3} .
$$


Assim o referencial $\left\{\widetilde{e}_{i}\right\}$ é dado em relação a base $\left\{e_{i}\right\}$ por

$$
\left[\begin{array}{c}
\widetilde{e}_{1} \\
\widetilde{e}_{2} \\
\widetilde{e}_{3}
\end{array}\right]=\left[\begin{array}{rrr}
1 & 0 & 0 \\
0 & -\epsilon_{2} \epsilon_{3} k & \epsilon_{3} \mu_{2} \\
0 & \mu_{2} & \epsilon_{3} k
\end{array}\right]=\left[\begin{array}{c}
e_{1} \\
e_{2} \\
e_{3}
\end{array}\right]
$$

em que $\mu_{2}$ é dado na expressão (4.7).

Fixemos os referenciais móveis $\left\{e_{i}\right\}$ e $\left\{\widetilde{e}_{i}\right\}$ adaptados as superfícies $\mathbb{X}$ e $\widetilde{\mathbb{X}}$ respectivamente e vamos relacionar seus co-referenciais móveis.

Primeiramente as condições 1) e 2) da Definição (4.2) nos permitem escrever

$$
\widetilde{\mathbb{X}}=\mathbb{X}+\lambda e_{1}
$$

Como $\lambda$ é constante, segue que $d \widetilde{\mathbb{X}}=d \mathbb{X}+\lambda d e_{1}$, cuja expressão em termos da base do co-referencial móvel é

$$
\widetilde{\epsilon}_{1} \widetilde{\omega}^{1} \widetilde{e}_{1}+\widetilde{\epsilon}_{2} \widetilde{\omega}^{2} \widetilde{e}_{2}=\epsilon_{1} \omega^{1} e_{1}+\epsilon_{2} \omega^{2} e_{2}+\lambda \epsilon_{2} \omega_{1}^{2} e_{2}+\lambda \epsilon_{3} \omega_{1}^{3} e_{3}
$$

em que $\widetilde{\omega}^{i}$ é o co-referencial móvel associado ao referencial $\left\{\widetilde{e}_{i}\right\}$ e é definido de acordo com a relação dada na Equação (2.14). Agora substituindo $\widetilde{e}_{i}$ pela relação 4.7) vem a seguinte expressão;

$$
\left(\widetilde{\epsilon}_{1} \widetilde{\omega}^{1}-\epsilon_{1} \omega^{1}\right) e_{1}+\left(-\widetilde{\epsilon}_{2} \epsilon_{2} \epsilon_{3} k \widetilde{\omega}^{2}-\epsilon_{2} \omega^{2}-\lambda \epsilon_{2} \omega_{1}^{2}\right) e_{2}+\left(\widetilde{\epsilon}_{2} \epsilon_{3} \mu_{2} \widetilde{\omega}^{2}-\lambda \epsilon_{3} \omega_{1}^{3}\right) e_{3}=0
$$

Usando a independência linear temos as seguintes relações entre os co-referenciais $\left\{\omega^{i}\right\}$ e $\left\{\widetilde{\omega}^{i}\right\}$,

$$
\begin{gathered}
\epsilon_{1} \omega^{1}=\widetilde{\epsilon}_{1} \widetilde{\omega}^{1}, \\
\omega^{2}+\lambda \omega_{1}^{2}=-\widetilde{\epsilon}_{2} \epsilon_{3} k \widetilde{\omega}^{2}, \\
\lambda \omega_{1}^{3}=\widetilde{\epsilon}_{2} \mu_{2} \widetilde{\omega}^{2} .
\end{gathered}
$$

Ainda podemos eliminar $\widetilde{\omega}^{2}$ das Equações 4.9 e 4.10 gerando

$$
\mu_{2} \omega^{2}=-\lambda\left(\epsilon_{3} k \omega_{1}^{3}-\mu_{2} \omega_{1}^{2}\right)
$$

Por outro lado, escrevendo as formas de conexão do co-referencial móvel associado a $\left\{\widetilde{\omega}^{i}\right\}$ 
na base do co-referencial móvel $\left\{\omega^{i}\right\}$ temos

$$
\begin{aligned}
\widetilde{\omega}_{1}^{3} & =\left\langle d \widetilde{e}_{1}, \widetilde{e}_{3}\right\rangle=\left\langle d e_{1}, \mu_{2} e_{2}+\epsilon_{3} k e_{3}\right\rangle \\
& =\mu_{2}\left\langle d e_{1}, e_{2}\right\rangle+\epsilon_{3} k\left\langle d e_{1}, e_{3}\right\rangle \\
& =\mu_{2} \omega_{1}^{2}+\epsilon_{3} k \omega_{1}^{3} \\
& =-\mu_{2} \lambda^{-1} \omega^{2}
\end{aligned}
$$

em que na última passagem usamos a Equação 4.11. Além disso

$$
\begin{aligned}
\widetilde{\omega}_{2}^{3} & =\left\langle d \widetilde{e}_{2}, \widetilde{e}_{3}\right\rangle=\left\langle-\epsilon_{2} \epsilon_{3} k d e_{2}+\epsilon_{3} \mu_{2} d e_{3}, \mu_{2} e_{2}+\epsilon_{3} k e_{3}\right\rangle \\
& =-\epsilon_{2} k^{2}\left\langle d e_{2}, e_{3}\right\rangle+\epsilon_{3} \mu_{2}^{2}\left\langle d e_{3}, e_{2}\right\rangle \\
& =-\epsilon_{2} k^{2} \omega_{2}^{3}+\epsilon_{3} \mu_{2}^{2} \omega_{3}^{2} \\
& =\left(-\epsilon_{2} k^{2}-\epsilon_{3} \mu_{2}^{2}\right) \omega_{2}^{3} \\
& =-\epsilon_{2} \epsilon_{3} \widetilde{\epsilon}_{3} \omega_{3}^{2}
\end{aligned}
$$

em que na última passagem substituímos $\mu_{2}$ via Equação (4.7).

Agora, partindo da Equação de Estrutura 2.20 para o co-referencial $\left\{\widetilde{\omega}^{i}\right\}$, e usando as Equações 4.12 e 4.13;

$$
\begin{aligned}
d \widetilde{\omega}_{1}^{2} & =\widetilde{\epsilon}_{3} \widetilde{\omega}_{1}^{3} \wedge \widetilde{\omega}_{3}^{2} \\
& =\widetilde{\epsilon}_{3}\left(-\mu_{2} \lambda^{-1} \omega^{2}\right) \wedge\left(-\epsilon_{2} \epsilon_{3} \widetilde{\epsilon}_{3} \omega_{3}^{2}\right) \\
& =-\epsilon_{2} \epsilon_{3} \mu_{2} \lambda^{-1} \omega^{2} \wedge \omega_{2}^{3} .
\end{aligned}
$$

Usando a Equação de Estrutura 2.19 para o co-referencial $\left\{\omega^{i}\right\}, \omega^{1} \wedge \omega_{1}^{3}+\epsilon_{2} \omega^{2} \wedge \omega_{2}^{3}=0$, temos que

$$
d \widetilde{\omega}_{1}^{2}=\epsilon_{1} \epsilon_{3} \mu_{2} \lambda^{-1} \omega^{1} \wedge \omega_{1}^{3}
$$

Agora usando as Equações 4.8 e 4.10 para re-escrever o lado direito em termos do coreferencial $\left\{\widetilde{\omega}^{i}\right\}$, temos,

$$
\begin{aligned}
d \widetilde{\omega}_{1}^{2} & =\epsilon_{1} \epsilon_{3} \mu_{2} \lambda^{-1}\left(\epsilon_{1} \widetilde{\epsilon}_{1} \widetilde{\omega}^{1}\right) \wedge\left(\widetilde{\epsilon}_{2} \mu_{2} \lambda^{-1} \widetilde{\omega}^{2}\right) . \\
& =\epsilon_{3} \widetilde{\epsilon}_{1} \widetilde{\epsilon}_{2} \mu_{2}^{2} \lambda^{-2} \widetilde{\omega}^{1} \wedge \widetilde{\omega}^{2} \\
& =-\epsilon_{3} \widetilde{\epsilon}_{3} \mu_{2}^{2} \lambda^{-2} \widetilde{\omega}^{1} \wedge \widetilde{\omega}^{2}
\end{aligned}
$$

onde na última igualdade usamos que $\widetilde{\epsilon}_{1} \widetilde{\epsilon}_{2} \widetilde{\epsilon}_{3}=-1$. 
Esta última expressão, juntamente com a Equação de Gauss 2.24, dada no Teorema 2.3 para o referencial $\left\{\widetilde{\omega}^{i}\right\}, d \widetilde{\omega}_{1}^{2}=-\widetilde{K} \widetilde{\omega}^{1} \wedge \widetilde{\omega}^{2}$, implica em

$$
\widetilde{K}=\epsilon_{3} \widetilde{\epsilon}_{3} \frac{\mu_{2}^{2}}{\lambda^{2}} \text {. }
$$

Em que $\mu_{2}$ é dado na Equação (4.7), isto é $\mu_{2}^{2}=\epsilon_{2} \widetilde{\epsilon}_{3}-\epsilon_{2} \epsilon_{3} k^{2}$. Deste modo vemos que $K$ é constante proporcional a constante $k$.

Analogamente ao que foi feito com a Equação 4.14 para o co-referencial $\left\{\omega^{i}\right\}$, podemos encontrar a relação de $K$ com os parâmetros $k$ e $\lambda$. De fato

$$
\begin{aligned}
d \omega_{1}^{2} & =\epsilon_{3} \omega_{1}^{3} \wedge \omega_{3}^{2} \\
& =\epsilon_{3}\left(\widetilde{\epsilon}_{2} \mu_{2} \lambda^{-1} \widetilde{\omega}^{2}\right) \wedge\left(-\epsilon_{2} \epsilon_{3} \widetilde{\epsilon}_{3} \widetilde{\omega}_{3}^{2}\right) \\
& =-\epsilon_{2} \widetilde{\epsilon}_{2} \widetilde{\epsilon}_{3} \mu_{2} \lambda^{-1}\left(\widetilde{\omega}^{2} \wedge \widetilde{\omega}_{3}^{2}\right) \\
& =-\epsilon_{2} \widetilde{\epsilon}_{2} \widetilde{\epsilon}_{3} \mu_{2} \lambda^{-1}\left(\widetilde{\epsilon}_{1} \widetilde{\epsilon}_{2} \widetilde{\omega}^{1} \wedge \widetilde{\omega}_{1}^{3}\right) \\
& =-\epsilon_{2} \widetilde{\epsilon}_{2} \widetilde{\epsilon}_{3} \mu_{2} \lambda^{-1}\left[\widetilde{\epsilon}_{1} \widetilde{\epsilon}_{2}\left(\widetilde{\epsilon}_{1} \epsilon_{1} \omega^{1}\right) \wedge\left(-\mu_{2} \lambda^{-1} \omega^{2}\right)\right] \\
& =\epsilon_{1} \epsilon_{2} \widetilde{\epsilon}_{3} \mu_{2}^{2} \lambda^{-2} \omega^{1} \wedge \omega^{2} \\
& =-\epsilon_{3} \widetilde{\epsilon}_{3} \mu_{2}^{2} \lambda^{-2} \omega^{1} \wedge \omega^{2}
\end{aligned}
$$

de modo que novamente a Equação de Gauss implica que

$$
K=\epsilon_{3} \widetilde{\epsilon}_{3} \frac{\mu_{2}^{2}}{\lambda^{2}},
$$

em que $\mu_{2}$ é dado na Equação 4.7 . Deste modo comparando com a curvatura $\widetilde{K}$, concluímos que as superfícies $\mathbb{X}$ e $\widetilde{\mathbb{X}}$ possuem a mesma curvatura e ambas idênticas a um valor constante.

E assim o teorema está demonstrado.

Teorema 4.3. (Teorema de Bäcklund em $\mathbb{L}^{3}$ ) Seja $\mathcal{B}: \mathbb{X} \rightarrow \widetilde{\mathbb{X}}$ uma congruência pseudoesférica entre duas superfícies parametrizadas regulares simples e não-degeneradas. Então

A: se a congruência $\mathcal{B}$ está no Caso $A$, a curvatura Gaussiana das duas superfícies é constante positiva igual $+\sinh ^{2} \phi / \lambda^{2}$.

B: se a congruência $\mathcal{B}$ está no Caso B, a curvatura Gaussiana das duas superfícies é constante positiva igual $+\sinh ^{2} \phi / \lambda^{2}$.

C: se a congruência $\mathcal{B}$ está no Caso $C$, a curvatura Gaussiana das duas superfícies é constante positiva igual $+\operatorname{sen}^{2} \phi / \lambda^{2}$. 
D: se a congruência $\mathcal{B}$ está no Caso D, a curvatura Gaussiana das duas superfícies é constante negativa igual $-\cosh ^{2} \phi / \lambda^{2}$.

Demonstração: Com a notação da demonstração do Teorema 4.2 temos que $\left\{e_{i}\right\}$ é um referencial móvel adaptado a superfície $\mathbb{X}$ com os sinais $\epsilon_{i}=\left\langle e_{i}, e_{i}\right\rangle$ e $\left\{\widetilde{e}_{i}\right\}$ é um referencial móvel adaptado a superfície $\widetilde{\mathbb{X}} \operatorname{com}$ os sinais $\widetilde{\epsilon}_{i}=\left\langle\widetilde{e}_{i}, \widetilde{e}_{i}\right\rangle$. De modo que a curvatura Gaussiana das superfícies $K$ e $\widetilde{K}$ são expressas de acordo com a equação

$$
K=\widetilde{K}=\epsilon_{3} \widetilde{\epsilon}_{3} \frac{\mu_{2}^{2}}{\lambda^{2}}
$$

em que $\mu_{2}^{2}=\epsilon_{2} \widetilde{\epsilon}_{3}-\epsilon_{2} \epsilon_{3} k^{2}, k=\langle N, \widetilde{N}\rangle$ é a constante não nula dada na condição 3) da Definição 4.2 e $\lambda=|\vec{r}|>0$ é a distância de congruência.

A: Neste caso $\mathbb{X}$ e $\widetilde{\mathbb{X}}$ são superfícies do tipo-espaço e a congruência pseudo-esférica é tipoespaço, então $\vec{r}$ é um vetor tipo-espaço e $\epsilon_{1}=\epsilon_{2}=\widetilde{\epsilon}_{1}=\widetilde{\epsilon}_{2}=1$ e $\epsilon_{3}=\widetilde{\epsilon}_{3}=-1$. Além disto fica definido, via Equação 4.4, um ângulo hiperbólico entre $e_{3}$ e $\widetilde{e}_{3}$. Assim

$$
k=\langle N, \tilde{N}\rangle=\tau \cosh (\phi),
$$

em que $\tau= \pm 1$ e $\phi \in(0,+\infty)$. Deste modo,

$$
K=\widetilde{K}=\frac{-1+\cosh ^{2}(\phi)}{\lambda^{2}}=\frac{\sinh ^{2}(\phi)}{\lambda^{2}}>0 .
$$

E assim concluímos que as duas superfícies têm a mesma curvatura constante positiva.

B: Neste caso $\mathbb{X}$ e $\widetilde{\mathbb{X}}$ são superfícies do tipo-tempo e a congruência pseudo-esférica é tipoespaço então $\epsilon_{1}=\widetilde{\epsilon}_{1}=1, \epsilon_{3}=\widetilde{\epsilon}_{3}=+1$ e $\epsilon_{2}=\widetilde{\epsilon}_{2}=-1$. Também, podemos tomar, via Equação 4.5,

$$
k=\tau \cosh (\phi),
$$

em que $\tau= \pm 1$ e $\phi \in(0,+\infty)$. Deste modo,

$$
K=\widetilde{K}=\frac{-1+\cosh ^{2}(\phi)}{\lambda^{2}}=\frac{\sinh ^{2}(\phi)}{\lambda^{2}}>0 .
$$

Assim garantimos que ambas superfícies têm mesma curvatura constante positiva.

C: Neste caso $\mathbb{X}$ e $\widetilde{\mathbb{X}}$ são superfícies do tipo-tempo e a congruência pseudo-esférica é tipotempo então $\epsilon_{1}=\widetilde{\epsilon}_{1}=-1, \epsilon_{3}=\widetilde{\epsilon}_{3}=+1$ e $\epsilon_{2}=\widetilde{\epsilon}_{2}=+1$. E também, podemos tomar, via Equação 4.6,

$$
k=\tau \cos (\phi),
$$


em que $\tau= \pm 1$ e $\phi \in(0, \pi)$. Deste modo,

$$
K=\widetilde{K}=\frac{1-\cos ^{2} \phi}{\lambda^{2}}=\frac{\operatorname{sen}^{2} \phi}{\lambda^{2}}>0 .
$$

Assim garantimos que ambas superfícies têm mesma curvatura constante positiva.

D: Neste caso $\mathbb{X}$ é uma superfície tipo-espaço e $\widetilde{\mathbb{X}}$ é uma superfícies tipo-tempo e a congruência pseudo-esférica é tipo-espaço, então $\epsilon_{1}=\epsilon_{2}=+1, \epsilon_{3}=-1, \widetilde{\epsilon}_{1}=\widetilde{\epsilon}_{3}=+1$, $\widetilde{\epsilon}_{2}=-1$. E também, podemos tomar, via Equação 4.6),

$$
k=\tau \sinh (\phi),
$$

em que $\tau= \pm 1$ e $\phi \in[0,+\infty)$. Deste modo,

$$
K=\widetilde{K}=-\frac{1+\sinh ^{2}(\phi)}{\lambda^{2}}=-\frac{\cosh ^{2}(\phi)}{\lambda^{2}}<0 .
$$

Assim garantimos que ambas superfícies têm mesma curvatura constante negativa.

\subsection{Condições de Integrabilidade}

Vimos na seção anterior que é possível definir o conceito de congruência pseudo-esférica no Espaço de Minkowski e tais congruências, se existirem, deverão ocorrer entre superfícies não-degeneradas com curvaturas constantes, conforme os casos de congruências $A, B, C$ e D. Nesta seção trataremos dos resultados de integrabilidade, isto é, dos resultados que garantem a existência de uma congruência pseudo-esférica. Mostraremos que cada uma das congruências $A, B, C$ e $D$ de fato existem e exibiremos as condições para que isto ocorra.

O termo "integrabilidade"é usado, em geral, para expressar a situação em que é possível garantir a existência de uma congruência pseudo-esférica. O emprego deste termo é justificado pelo processo que é usado para garantir a existência de uma congruência, na qual passa por integrar, isto é obter soluções, de um sistema certo sistema de EDP's. Conforme veremos os sistemas de EDP's que aparecem podem ser integrados por uma versão do Teorema de Frobenius, qual vamos tratar agora.

Primeiramente assumiremos o Teorema de Picard qual garante condições necessárias e suficientes para existência e unicidade, pelo menos locais, de um problema de valor inicial. Tal teorema é encontrado em vários textos sobre Equações Diferenciais Ordinárias, como por exemplo no Teorema 3.1 página 88 de [8]. 
Lema 4.1. (Teorema de Picard) Seja $f: U \subset \mathbb{R}^{2} \rightarrow \mathbb{R}$ uma função de $(x, y) \in U$ conjunto aberto de $\mathbb{R}^{2}$ tal que $f$ e $f_{y}$ são funções contínuas em $U$. Então, dado $\left(x_{0}, y_{0}\right) \in U$ existe um intervalo aberto $I \subset \mathbb{R}$ contendo $x_{0}$ e uma função $u: I \subset \mathbb{R} \rightarrow \mathbb{R}$ satisfazendo $\frac{d u}{d x}(x)=f(x, u(x))$ e uma condição inicial $u\left(x_{0}\right)=u_{0} \in \mathbb{R}$. Além disso qualquer outra solução $\widetilde{u}(x)$ da equação $\frac{d \widetilde{u}(x)}{d x}=f(x, \widetilde{x})$ satisfazendo $\widetilde{u}\left(x_{0}\right)=x_{0}$ é tal que $\widetilde{u}(x)=u(x)$ em $I$.

Este Lema será usado repetidas vezes para demonstrar o Teorema de Frobenius. Além disso o Lema vale, em particular, para funções diferenciáveis (isto é, funções de classe $C^{\infty}$ ).

Teorema 4.4. (Teorema de Frobenius) Sejam A, B funções reais diferenciáveis, $u: U \subset$ $\mathbb{R}^{2} \rightarrow \mathbb{R}$ com $U$ um subconjunto aberto de $\mathbb{R}^{2} e\left(x_{0}, y_{0}\right) \in U$. Então o sistema em $u=u(x, y)$

$$
\left\{\begin{array}{l}
u_{x}=A(x, y, u) \\
u_{y}=B(x, y, u)
\end{array}\right.
$$

com $u\left(x_{0}, y_{0}\right)=u_{0} \in \mathbb{R}$ admite solução em uma vizinhança de $\left(x_{0}, y_{0}\right)$ se, e somente se, verifica-se

$$
\frac{\partial A}{\partial y}+\frac{\partial A}{\partial u} B=\frac{\partial B}{\partial x}+\frac{\partial B}{\partial u} A
$$

dita condição de integrabilidade.

O papel deste teorema é condicionar a existência local de soluções do sistema (4.16) com uma certa condição (4.17). Existem versões bem mais gerais para o Teorema de Frobenius que podem ser entradas nos textos de Formas Diferenciais, como por exemplo [14] Teorema 1.3.4 página 11. Entretanto, vamos demonstrar esta versão, que será suficiente.

Demonstração: Esta demostração é baseada nos comentários que seguem o Exemplo 1.2.3 da página 6 de [14].

Primeiramente, suponhamos que $u$ seja uma solução diferenciável do sistema 4.16). Deste modo pelo Teorema de Cauchy $\left(u_{x}\right)_{y}=\left(u_{y}\right)_{x}$, que implica em

$$
\begin{aligned}
\frac{\partial u_{x}}{\partial y} & =\frac{\partial u_{y}}{\partial x} \\
\frac{\partial A}{\partial y}+\frac{\partial A}{\partial u} \frac{\partial u}{\partial y} & =\frac{\partial B}{\partial x}+\frac{\partial B}{\partial u} \frac{\partial u}{\partial x} \\
\frac{\partial A}{\partial y}+\frac{\partial A}{\partial u} B & =\frac{\partial B}{\partial x}+\frac{\partial B}{\partial u} A
\end{aligned}
$$

e assim vale a Equação 4.17).

Reciprocamente, assumamos que valha a Equação 4.17) e consideremos o sistema 4.16 com a condição inicial $u\left(x_{0}, y_{0}\right)=u_{0}$. A primeira equação deste sistema induz uma equação 
diferencial ordinária, digamos

$$
\frac{d \mu}{d x}(x)=A\left(x, y_{0}, \mu(x)\right) ; \quad \mu\left(x_{0}\right)=u_{0} .
$$

Aplicando o Lema 4.1), existem um intervalo $I \subset \mathbb{R}$ aberto de $\mathbb{R}$ e uma única função $\mu(x)$, solução deste sistema satisfazendo $\mu\left(x_{0}\right)=u_{0}$.

Para cada $x^{\prime} \in I$ considere as seguintes equações diferenciais ordinárias na variável $y$,

$$
\frac{d \xi^{\left\{x^{\prime}\right\}}}{d y}(y)=B\left(x^{\prime}, y, \xi^{\left\{x^{\prime}\right\}}\right) ; \quad \xi^{\left\{x^{\prime}\right\}}\left(y_{0}\right)=\mu\left(x^{\prime}\right),
$$

em que, nesta notação, o subescrito $\left\{x^{\prime}\right\}$ indica que o sistema, na função $\xi^{\left\{x^{\prime}\right\}}(y)$ de $y$, é tomada para $x^{\prime} \in I$ fixado. Aplicando-se o Lema 4.1 para cada uma destas equações diferenciais ordinárias, garantimos, para cada $x^{\prime}$ uma única solução $\xi^{\left\{x^{\prime}\right\}}$ definida em um aberto $J^{\left\{x^{\prime}\right\}} \subset \mathbb{R}$, que depende de cada valor $x^{\prime}$ fixado, satisfazendo a condição inicial $\xi^{\left\{x^{\prime}\right\}}=$ $\mu\left(x^{\prime}\right)$.

Definimos então a função $u(x, y)=\xi^{\{x\}}$ em uma vizinhança de $\left(x_{0}, y_{0}\right)$, com $x \in I$ e $y \in J^{\left\{x_{0}\right\}}$ candidata a solução do sistema. Por construção, $u$ automaticamente satisfaz a segunda equação do sistema 4.16 para cada $x, y$, isto é,

$$
\frac{\partial u}{\partial y}=\frac{d \xi^{\{x\}}}{d y}=B\left(x, y, \xi^{\{x\}}\right)=B(x, y, u(x)),
$$

e também para todo $x \in I$ e para $y=y_{0}$, temos que $u(x, y)$ é solução da primeira equação, isto é,

$$
\frac{\partial u}{\partial x}\left(x, y_{0}\right)=\frac{\partial \xi^{\{x\}}\left(y_{0}\right)}{\partial x}=\frac{d \mu(x)}{d x}=A\left(x, y_{0}, \mu\right)=A\left(x, y_{0}, u\left(x, y_{0}\right)\right) .
$$

Assim nos resta mostrar que a candidata a solução $u$ satisfaz a primeira equação do sistema para todos os pontos $(x, y)$ em alguma vizinhança de $\left(x_{0}, y_{0}\right)$. Para tanto basta mostrar que a função $R(x, y)=\frac{\partial u}{\partial x}-A$ é identicamente nula em tal vizinhança de $\left(x_{0}, y_{0}\right)$. Perceba que nos pontos $\left(x, y_{0}\right)$ já temos que $R\left(x, y_{0}\right)=0$.

Utilizando a hipótese de que vale a condição 4.17, temos que $R$ é solução do seguinte 
sistema $\frac{\partial R}{\partial y}=\frac{\partial B}{\partial u} R$. De fato

$$
\begin{aligned}
\frac{\partial R}{\partial y} & =\frac{\partial}{\partial y} \frac{\partial u}{\partial x}-\frac{\partial}{\partial y} A \\
& =\frac{\partial}{\partial x} \frac{\partial u}{\partial y}-\frac{\partial A}{\partial y}-\frac{\partial A}{\partial u} \frac{\partial u}{\partial y} \\
& =\frac{\partial}{\partial x} B-\left(\frac{\partial A}{\partial y}+\frac{\partial A}{\partial u} B\right) \\
& =\frac{\partial B}{\partial x}+\frac{\partial B}{\partial u} \frac{\partial u}{\partial x}-\left(\frac{\partial B}{\partial x}+\frac{\partial B}{\partial u} A\right) \\
& =\frac{\partial B}{\partial u}\left(\frac{\partial u}{\partial x}-A\right) \\
& =\frac{\partial B}{\partial u} R .
\end{aligned}
$$

Já sabemos que nos pontos $\left(x, y_{0}\right) \operatorname{com} x \in I$ temos $R\left(x, y_{0}\right)=0$. Assim seja a função real $h^{\{x\}}(y)=R(x, y)$ na variável $y$ para algum $x \in I$ fixado. Como $R$ é solução de 4.18,, temos que $h^{\{x\}}$ é solução da seguinte equação diferencial ordinária

$$
\frac{d}{d y} h^{\{x\}}(y)=\frac{\partial B}{\partial u} h^{\{x\}}
$$

Isto é, omitindo $x$ e denotando $g(y)=\frac{\partial B}{\partial u}(x, y)$, temos que

$$
h^{\prime}(y)=g(y) h(y)
$$

que no ponto $y=y_{0}$ se anula.

Consequentemente $h^{\prime \prime}(y)=g^{\prime}(y) h(y)+g(y) h^{\prime}(y)$ se anula em $y=y_{0}$. Prosseguindo por indução, temos que a derivada de todas as ordens de $h$ se anula em $y_{0}$. Deste modo, utilizando a expansão de Taylor em séries de potências, temos que $h(y)$ é nula em uma vizinhança de $y_{0}$. Consequentemente $R(x, y)$ se anula em uma vizinhança de $\left(x_{0}, y_{0}\right)$ e assim temos que a candidata $u(x, y)$ é de fato solução do sistema 4.16.

E o teorema está demonstrado.

Visto isso, estamos em condições de buscar as condições suficientes para existência de congruências pseudo-esféricas. Abordaremos primeiro o caso clássico para superfícies no espaço Euclidiano qual servirá de inspiração para o caso generalizado no espaço de Minkowski.

\subsubsection{Caso Clássico}

Nesta seção demonstraremos o resultado clássico de integrabilidade, isto é, o resultado que nos garante as condições em que é possível, partindo de uma certa superfície, obter uma congruência pseudo-esférica. 
Teorema 4.5. (de Integrabilidade) Seja $X: U \subset \rightarrow \mathbb{E}^{3}$ uma superfície parametrizada regular simples com curvatura Gaussiana $K=-\frac{\sin ^{2} \phi}{\lambda^{2}}, \phi \in(0, \pi)$ e $\lambda>0$ constantes. Então para cada direção não principal $e_{0}(q) \in T_{q} X$ existe uma superfície $\widetilde{X}$ relacionada com $X$ por uma congruência pseudo-esférica tendo e $e_{0}$ como direção de congruência e parâmetros $\lambda$ e $\phi$.

A idéia para demonstrar este teorema é construir um referencial móvel adequado sobre a superfície $X$ e partindo deste referencial obter uma condição para que $\widetilde{X}=X+\lambda e_{0}$ seja uma superfície parametrizada regular. Veremos que tal condição é equivalente ao processo analítico de integrar um sistema de duas EDP's que coincide precisamente com o tipo de sistema 4.16 considerado no Teorema de Frobenius 4.4.

Demonstração(do Teorema de Integrabilidade): Suponhamos, por simplicidade, que $\lambda^{2}=\sin ^{2} \phi$, isto é, estamos supondo que a curvatura Gaussiana de $X$ é $K=-1$. De modo podemos supor que $X(u, v)$ está parametrizada pelas coordendas assintóticas de Tchebyschev $(u, v)$, introduzidas na Definição (3.1). Seja $\left\{e_{1}, e_{2}, e_{3}\right\}$ o referencial móvel de Chebyschev (dado também na Definição 3.1) associado a superfície $X$, isto é, o co-refenencial móvel é dado por

$$
\begin{array}{r}
\omega^{1}=\cos \frac{\alpha}{2} d u, \quad \omega^{2}=\sin \frac{\alpha}{2} d v, \\
\omega_{1}^{2}=\frac{1}{2} \alpha_{v} d u+\frac{1}{2} \alpha_{u} d v, \\
\omega_{1}^{3}=\sin \frac{\alpha}{2} d u, \quad \omega_{1}^{3}=-\cos \frac{\alpha}{2} d v,
\end{array}
$$

em que $\alpha \in(0, \pi)$ é uma solução da Equação de sine-Gordon $\alpha_{u u}-\alpha_{v v}=\sin \alpha$.

Mostrar que existe uma congruência pseudo-esférica entre $X$ e uma superfície $\widetilde{X}$ é equivalente a mostrar que existe uma função, digamos $\theta=\theta(u, v) \in\left(0, \frac{\pi}{2}\right)$, tal que

$$
\widetilde{X}=X+\lambda\left(\cos \theta e_{1}+\sin \theta e_{2}\right)
$$

seja uma superfície parametrizada regular. Note que, neste caso, $e_{0}=\cos \theta e_{1}+\sin \theta e_{2}$ será a direção de congruência e que também não será uma direção principal $e_{1}$ ou $e_{2}$ de $X$.

Usando as relações,

$$
d(\sin \theta)=\cos \theta \theta_{u} d u+\cos \theta \theta_{v} d v=\cos \theta d \theta
$$

e

$$
d(\cos \theta)=-\sin \theta d \theta .
$$

diferenciando exteriormente a expressão 4.20], obtemos

$$
d \tilde{X}=d X+\lambda\left(-\sin \theta d \theta e_{1}+\cos \theta d e_{1}+\cos \theta d \theta e_{2}+\sin \theta d e_{2}\right) .
$$


Usando que $d X=\omega^{1} e_{1}+\omega^{2} e_{2}$ e $d e_{j}=\omega_{j}^{1} e_{1}+\omega_{j}^{2} e_{2}+\omega_{j}^{3} e_{3}$, em que o co-referencial móvel e as formas de conexão são dadas por (4.19), temos

$$
\begin{aligned}
d \widetilde{X}= & {\left[\cos \frac{\alpha}{2} d u-\lambda \sin \theta d \theta-\lambda \sin \theta\left(\frac{1}{2} \alpha_{v} d u+\frac{1}{2} \alpha_{u} d v\right)\right] e_{1}+} \\
& +\left[\sin \frac{\alpha}{2} d v+\lambda \cos \theta d \theta+\lambda \cos \theta\left(\frac{1}{2} \alpha_{v} d u+\frac{1}{2} \alpha_{u} d v\right)\right] e_{2}+ \\
& +\left[\lambda \cos \theta \sin \frac{\alpha}{2} d u-\lambda \sin \theta \cos \frac{\alpha}{2} d v\right] e_{3} .
\end{aligned}
$$

Por outro lado, definindo

$$
\widetilde{N}:=\cos \phi N+\sin \phi r_{\theta}^{\perp},
$$

em que $\phi$ é a constante de congruência, $r_{\theta}^{\perp}=\sin \theta e_{1}-\cos \theta e_{2}$ e $N$ é a normal da superfície $X$. Podemos impor que a função incógnita $\theta$ satisfaça a relação $\langle d \widetilde{X}, \widetilde{N}\rangle_{\mathbb{E}^{3}}$, isto é, pedimos que $\theta$ seja uma função tal que,

$$
\begin{aligned}
0= & -\lambda \sin \phi d \theta-\lambda \sin \phi\left(\frac{1}{2} \alpha_{v} d u+\frac{1}{2} \alpha_{u} d v\right)+ \\
& +\sin \phi\left(\cos \frac{\alpha}{2} \sin \theta d u-\sin \frac{\alpha}{2} \cos \theta d v\right)+ \\
& +\lambda \cos \phi\left(\sin \frac{\alpha}{2} \cos \theta d u-\cos \frac{\alpha}{2} \sin \theta d v\right) .
\end{aligned}
$$

Usando que $\lambda=\sin \phi$, com $\phi \in(0, \pi)$, e fazendo

$$
\theta=\frac{\widetilde{\alpha}}{2}
$$

para alguma função incógnita $\widetilde{\alpha}$, temos que a condição 4.23 , , expressa em termos de $\widetilde{\alpha}$, é dada por

$$
\begin{aligned}
0= & \left(-\frac{1}{2} \sin \phi \widetilde{\alpha}_{u}-\frac{1}{2} \sin \phi \alpha_{v}+\cos \frac{\alpha}{2} \sin \frac{\widetilde{\alpha}}{2}+\cos \phi \sin \frac{\alpha}{2} \cos \frac{\widetilde{\alpha}}{2}\right) d u+ \\
& \left(-\frac{1}{2} \sin \phi \widetilde{\alpha}_{v}-\frac{1}{2} \sin \phi \alpha_{u}-\sin \frac{\alpha}{2} \cos \frac{\widetilde{\alpha}}{2}-\cos \phi \cos \frac{\alpha}{2} \sin \frac{\widetilde{\alpha}}{2}\right) d v .
\end{aligned}
$$

Usando a independência linear entre as formas $d u$ e $d v$, temos que a função incógnita $\widetilde{\alpha}$ satisfaz esta equação, se, e somente se, ela satisfaz o sistema

$$
\left\{\begin{array}{l}
\frac{1}{2} \sin \phi\left(\widetilde{\alpha}_{u}+\alpha_{v}\right)=\cos \phi \sin \frac{\alpha}{2} \cos \frac{\widetilde{\alpha}}{2}+\cos \frac{\alpha}{2} \sin \frac{\widetilde{\alpha}}{2} \\
\frac{1}{2} \sin \phi\left(\widetilde{\alpha}_{v}+\alpha_{u}\right)=-\cos \phi \cos \frac{\alpha}{2} \sin \frac{\widetilde{\alpha}}{2}-\sin \frac{\alpha}{2} \cos \frac{\widetilde{\alpha}}{2}
\end{array}\right.
$$

que é precisamente um sistema do tipo de Frobenius (4.16) sobre a incónita $\widetilde{\alpha}$. Utilizando o Teorema de Frobenius (4.4), podemos ver que a condição de integrabilidade (4.17) é justamente a equação de sine-Gordon,

$$
\alpha_{u u}-\alpha_{v v}=\sin \alpha
$$


Uma vez que as coordenadas $(u, v)$ são coordenadas de Tchebyschef em $X$, temos que $\alpha$ é automaticamente solução da equação de sine-Gordon, de modo que a condição de compatibilidade do Teorema de Frobenius é satisfeita e assim o sistema (4.24) é completamente integrável sobre $\widetilde{\alpha}$ e o Teorema está demonstrado.

Corolário 4.1. (da demonstração do Teorema (4.5)) Suponha que $X(u, v)$ e $\tilde{X}(u, v)$ estejam relacionadas por uma congruência pseudo-esférica, com parâmetros $\lambda$, $\phi$ e direção de congruência que não é uma direção principal de X. Então as coordenadas de Tchebyschef $(u, v)$ em $X$ também serão coordenadas de Tchebyschef em $\widetilde{X}$. Além disso $\widetilde{\alpha}$ é uma solução da equação de sine-Gordon.

Demonstração: Primeiramente considerando o sistema (4.24), podemos derivar parcialmente a primeira equação em relação $u$ e a segunda equação em relação a $v$, tomar a diferença e obter,

$$
\left(\widetilde{\alpha}_{u u}-\widetilde{\alpha}_{u u}\right)+\alpha_{v u}-\alpha_{u v}=\sin \widetilde{\alpha} .
$$

Desse modo a diferenciabilidade de $\alpha$ é equivalente a função $\widetilde{\alpha}$ satisfazer a equação de sineGordon.

Considere agora a relação 4.21). Reescrevendo em termos de $d u$ e $d v$ e usando que $d \theta=\frac{1}{2} \widetilde{\alpha}_{u} d u+\frac{1}{2} \widetilde{\alpha}_{v} d v$ teremos

$$
\begin{aligned}
d \widetilde{X}= & {\left[\left(\cos \frac{\alpha}{2}-\lambda \sin \widetilde{\alpha}\left(\frac{\widetilde{\alpha}_{u}}{2}+\frac{\alpha_{v}}{2}\right)\right) e_{1}+\lambda \cos \frac{\widetilde{\alpha}}{2}\left(\frac{\widetilde{\alpha}_{u}}{2}+\frac{\alpha_{v}}{2}\right) e_{2}+\right.} \\
& \left.+\left(\lambda \cos \frac{\widetilde{\alpha}}{2} \sin \frac{\alpha}{2}\right) e_{3}\right] d u+ \\
+ & {\left[-\lambda \sin \frac{\widetilde{\alpha}}{2}\left(\frac{\widetilde{\alpha}}{2}-\frac{\alpha_{u}}{2}\right) e_{1}+\left(\sin \frac{\alpha}{2}+\lambda \cos \frac{\widetilde{\alpha}}{2}\left(\frac{\widetilde{\alpha}_{v}}{2}+\frac{\alpha_{u}}{2}\right)\right) e_{2}+\right.} \\
& \left.+\left(-\lambda \sin \frac{\widetilde{\alpha}}{2} \cos \frac{\alpha}{2}\right) e_{3}\right] d v
\end{aligned}
$$

Utilizando as expressões (4.24 juntamente $\operatorname{com} \lambda=\sin \phi$ temos que

$$
d \widetilde{X}=\widetilde{A} d u \widetilde{e}_{1}+\widetilde{B} d v \widetilde{e}_{2}
$$


em que

$$
\begin{aligned}
\widetilde{A}= & \cos \frac{\widetilde{\alpha}}{2}, \\
\widetilde{B}= & \sin \frac{\widetilde{\alpha}}{2}, \\
\widetilde{e}_{1}= & \left(\cos \frac{\alpha}{2} \cos \frac{\widetilde{\alpha}}{2}-\cos \phi \sin \frac{\alpha}{2} \sin \frac{\widetilde{\alpha}}{2}\right) e_{1}+ \\
& +\left(\cos \frac{\alpha}{2} \sin \frac{\widetilde{\alpha}}{2}+\cos \phi \sin \frac{\alpha}{2} \cos \frac{\widetilde{\alpha}}{2}\right) e_{2}+\sin \phi \sin \frac{\alpha}{2} e_{3}, \\
\widetilde{e}_{2}= & \left(\sin \frac{\alpha}{2} \cos \frac{\widetilde{\alpha}}{2}+\cos \phi \sin \frac{\alpha}{2} \cos \frac{\widetilde{\alpha}}{2}\right) e_{1}+ \\
& +\left(\sin \frac{\alpha}{2} \sin \frac{\widetilde{\alpha}}{2}-\cos \phi \cos \frac{\alpha}{2} \cos \frac{\widetilde{\alpha}}{2}\right) e_{2}-\sin \phi \cos \frac{\alpha}{2} e_{3} .
\end{aligned}
$$

Assim por definição do co-referencial móvel, $d \widetilde{X}=\widetilde{\omega}^{1} \widetilde{e}_{1}+\widetilde{\omega}^{2} \widetilde{e}_{2}$, nos permite identificar,

$$
\begin{aligned}
\widetilde{\omega}^{1} & =\widetilde{A} d u=\cos \frac{\widetilde{\alpha}}{2} d u \\
\widetilde{\omega}^{2} & =\widetilde{B} d v=\sin \frac{\widetilde{\alpha}}{2} d v .
\end{aligned}
$$

Por outro lado, repetindo o mesmo argumento com $d \widetilde{N}$ na equação 4.22 e utilizando a definição das formas de conexão $d \widetilde{N}=\widetilde{\omega}_{3}^{1} \widetilde{e}_{1}+\widetilde{\omega}_{3}^{2} \widetilde{e}_{2}$, podemos identificar

$$
\begin{gathered}
\widetilde{\omega}_{3}^{1}=-\sin \frac{\widetilde{\alpha}}{2} d u, \\
\widetilde{\omega}_{3}^{2}=\cos \frac{\widetilde{\alpha}}{2} d v .
\end{gathered}
$$

De acordo com a Definição (3.1), temos que $(u, v)$ também são parâmetros de Tchebyschev em $\widetilde{X}$. Como consequência, assim como na Definição (3.1), a função $\widetilde{\alpha}$ representa o ângulo que as linhas assintóticas fazem com as linhas de curvatura.

Definição 4.3. No contexto do Teorema de Integrabilidade 4.5), a superfície $\widetilde{X}$ é dita Transformação de Bäcklund da superfície $X$ e no contexto do Corolário (4.1) a equação $\widetilde{\alpha}$, solução do sistema (4.16), é dita Transformação de Bäcklund da função $\alpha$.

Por último, juntando os resultados de Integrabilidade desta seção com os resultados obtidos na seção (3.1.1), obtemos o seguinte Teorema.

Teorema 4.6. Partindo de uma solução $0<\alpha<\pi$ da equação de sine-Gordon, existe uma família a dois parâmetros de soluções da equação de sine-Gordon. 
Demonstração: De fato, dada uma solução $\alpha$ da equação de sine-Gordon podemos aplicar o Teorema (3.1) e obter uma superfície $X$ com curvatura Gaussiana constante negativa, digamos $K=-\frac{\sin ^{2} \alpha}{\lambda^{2}}$, para algum par de constantes $\lambda>0$ e $0<\phi<\pi$. A partir desta superfície, podemos aplicar o Teorema de Integrabilidade 4.5 e obter uma família a dois parâmetros de superfícies (em que um dos parâmetros é uma direção não principal do espaço tangente de $X$ e outro parâmetro é $\lambda$ ou $\phi$ que depende da relação com $K$ ).

Para cada superfície desta família podemos aplicar o Teorema de Bäcklund 4.1) e garantir que esta superfície está nas condições do Teorema (3.1) de modo que podemos fazer corresponder a cada uma destas superfícies uma nova solução da equação de sine-Gordon e obter assim uma família a dois parâmetros de soluções.

Alternativamente (e equivalentemente) ao invés de aplicarmos o Teorema de Bäcklund 4.1. podemos usar o Corolário 4.1 e garantir que a cada uma destas superfícies a solução $\widetilde{\alpha}$ integral do sistema (4.16) é justamente uma solução da equação de equação de sine-Gordon. Deste modo obtemos uma família a dois parâmetros de soluções da equação de sine-Gordon.

Estas duas últimas passagens são equivalentes pelo fato de que a solução $\widetilde{\alpha}$ integral do sistema (4.16) coincide precisamente com a solução da Equação de Gauss (3.18) durante a demonstração do Teorema (3.1), pois nas duas situações a superfície é descrita pela pelas coordenadas de Tchebyschev., para superfícies no espaço de Minkowski, para superfícies no espaço de Minkowski

\subsubsection{Caso Generalizado}

Vamos tratar nesta seção dos resultados de Integrabilidade para Transformações de Bäcklund no Espaço de Minkowski. Mais precisamente, vamos demonstrar o seguinte Teorema.

Teorema 4.7. (de Integrabilidade Generalizado) Seja $\mathbb{X}$ uma superfície parametrizada regular não-degenerada com curvatura Gaussiana constante não nula. Então existe uma superfície $\widetilde{X}$ em cada um dos oito casos abaixo,

A: $\mathbb{X}$ tipo-espaço, não umbílica, com $K=\sinh ^{2} \phi / \lambda^{2}>0$ e $\triangle \geq 0$,

$B_{1}: \mathbb{X}$ tipo-tempo com $K=\sinh ^{2} \phi / \lambda^{2}>0$ e $\triangle>0$,

$B_{2}: \mathbb{X}$ tipo-tempo, não umbílica, com $K=\sinh ^{2} \phi / \lambda^{2}>0$ e $\triangle=0$,

$B_{3}: \mathbb{X}$ tipo-tempo com $K=\sinh ^{2} \phi / \lambda^{2}>0$ e $\triangle<0$,

$C_{1}: \mathbb{X}$ tipo-tempo com $K=\operatorname{sen}^{2} \phi / \lambda^{2}>0$ e $\triangle>0$,

$C_{2}: \mathbb{X}$ tipo-tempo, não umbílica, com $K=\operatorname{sen}^{2} \phi / \lambda^{2}>0$ e $\triangle=0$, 
$C_{3}: \mathbb{X}$ tipo-tempo com $K=\operatorname{sen}^{2} \phi / \lambda^{2}>0$ e $\triangle<0$,

$D: \mathbb{X}$ tipo-espaço, não umbílica, com $K=-\cosh ^{2} \phi / \lambda^{2}<0$ e $\triangle \geq 0$.

Existe uma congruência pseudo-esférica do tipo $A, B, C$ e $D$, de parâmetros $\lambda$ e $\phi$ para cada direção não principal do plano tangente.

A demonstração deste Teorema é feita considerando cada caso separadamente, e foi demonstrado ao longo de 1980 até 2003 nos Artigos [15], [19], 21] e [12]. Traremos estas demonstrações no final da seção. Antes vamos buscar alguns Corolários deste Teorema que são análogos ao Corolário (4.1) do Teorema de Integrabilidade Clássico (4.5).

Corolário 4.2. (do Teorema (4.7)) Partindo de uma solução, não trivial, é possivel obter uma familia a dois parâmetros de soluções de cada uma das seguintes EDP's,

A: Sine-Gordon $\alpha_{u u}-\alpha_{v v}=\sin \alpha$,

$B_{1}, C_{1}$ : Sinh-Gordon $\alpha_{u u}-\alpha_{v v}=-\sinh \alpha$,

$B_{2}, C_{2}$ : Liouville $\alpha_{u v}=-\frac{1}{2} e^{2 \alpha}$,

$B_{3}, C_{3}$ : Cosh-Gordon $\alpha_{u v}=\cosh \alpha$.

Corolário 4.3. (do Teorema (4.7)) Existe, pelo caso D, uma relação biunívoca entre as soluções da equação de sine-Laplace $\left(\alpha_{u u}+\alpha_{v v}=\sin \alpha\right)$ e Sinh-Laplace $\left(\alpha_{u u}+\alpha_{v v}=\sinh \alpha\right)$. Além disso, de uma solução não nula de uma destas equações é possivel obter uma família a dois parâmetros de soluções da outra.

Veremos agora a demonstração do Teorema 4.7). A demonstração é feita considerando separadamente os casos e em cada um deles a demonstração segue os mesmos passos que a demonstração do Teorema Clássico (4.5). Para tal consideramos sobre a superfície Coordenadas de Tchebyschev juntamente com um referencial móvel apropriado e partindo deste referencial móvel expressamos uma condição de existência de uma superfície congruente e então usar o Teorema de Frobenius 4.4 para obter tal superfície.

\section{Demonstração do Teorema de Integrabilidade:}

Consideremos inicialmente o caso $A$.

Seja $\mathbb{X}$ superfície tipo-espaço, com curvatura Gaussiana constante igual a $K=1$, tal que o discriminante do Polinômio Característico (1.21) é $\triangle \geq 0$. Supondo que $\mathbb{X}$ não tenha pontos umbílicos podemos considerar que $\mathbb{X}(u, v)$ está parametrizada por coordenadas $(u, v)$ de Tchebyschev como no Caso I da Seção 3.2 . Assim consideramos o Referencial Móvel de Tchebyschev $\left\{e_{1}, e_{2}, e_{3}\right\}$ com o Co-Referencial Móvel e as formas de conexão dados pelas 
Expressões (3.26)- 3.30), isto é

$$
\begin{aligned}
\omega^{1} & =\cos (\alpha / 2) d u \\
\omega^{2} & =\sin (\alpha / 2) d v \\
\omega_{1}^{2} & =\frac{1}{2} \alpha_{v} d u+\frac{1}{2} \alpha_{u} d v, \\
\omega_{1}^{3} & =\sin (\alpha / 2) d u \\
\omega_{2}^{3} & =-\cos (\alpha / 2) d v,
\end{aligned}
$$

com $0<\alpha(u, v)<\pi$ uma solução da equação de sine-Gordon.

Seguindo a idéia da demonstração do Teorema de Integrabilidade Clássico (4.5), buscamos uma função $0<\theta<\pi / 2$ tal que,

$$
\begin{array}{r}
\widetilde{\mathbb{X}}=\mathbb{X}+\lambda\left(\cos \theta e_{1}+\sin \theta e_{2}\right) \\
\widetilde{N}=\sinh \phi\left(-\sin \theta e_{1}+\cos \theta e_{2}\right)+\cosh \phi e_{3}
\end{array}
$$

em que, $\lambda$ é a distância de congruência, $\phi$ é o ângulo hiperbólico de congruência definido pela Equação 4.4 tais que $\lambda=\sinh \phi, \widetilde{N}$ é a expressão para o vetor normal a superfície $\mathbb{X}$. Perceba que, neste caso, a direção de congruência será dada pelo vetor $\cos \theta e_{1}+\sin \theta e_{2}$ tipo-espaço (uma vez que procuramos uma congruência tipo-espaço), e $\widetilde{N}$ define um vetor tipo-tempo, isto é, $\widetilde{\mathbb{X}}$ será uma superfície tipo-espaço, o que é precisamente uma Congruência Pseudo-Esférica Tipo $A$.

Utilizando que $d \mathbb{X}=\omega^{1} e_{1}+\omega^{2} e_{2}$ e $d e_{j}=\omega_{j}^{1} e_{1}+\omega_{j}^{2} e_{2}+\omega_{j}^{3} e_{3}$, a condição $\langle d \widetilde{\mathbb{X}}, \widetilde{N}\rangle=0$ juntamente com a independência linear de $d u$ e $d v$, temos

$$
\left\{\begin{array}{l}
\frac{\lambda}{2}\left(\widetilde{\alpha}_{u}+\alpha_{v}\right)=\sin \frac{\widetilde{\alpha}}{2} \cos \frac{\alpha}{2}+\cosh \phi \cos \frac{\widetilde{\alpha}}{2} \sin \frac{\alpha}{2} \\
\frac{\lambda}{2}\left(\widetilde{\alpha}_{v}+\alpha_{u}\right)=-\cos \frac{\widetilde{\alpha}}{2} \sin \frac{\alpha}{2}-\cosh \phi \sin \frac{\widetilde{\alpha}}{2} \cos \frac{\alpha}{2}
\end{array}\right.
$$

Em que denotamos $\frac{\widetilde{\alpha}}{2}=\theta$. Este é um Sistema de Equações Diferenciais Parciais do tipo de Frobenius em relação a função incógnita $\widetilde{\alpha}(u, v)$. Utilizando o Teorema de Frobenius (4.4), temos que este sistema é integrável uma vez que a condição de compatibilidade é exatamente $\alpha_{u u}-\alpha_{v v}=\sin \alpha$. Assim existe a função $\widetilde{\alpha}(u, v)$ e consequentemente a equação (4.34) define uma superfície $\widetilde{X}$ que será congruente a superfície $\mathbb{X}$.

O que demonstra o Teorema para o Caso A.

Para os demais casos a demonstração é análoga, basta considerar que a superfície $\mathbb{X}(u, v)$ esteja parametrizada respectivamente por:

Casos $B_{1}$ e $C_{1}$ Coordenadas de Tchebyschef IV, 
Casos $B_{2}$ e $C_{2}$ Coordenadas Nulas de McNertney com parâmetro $\mu=\frac{1}{2} e^{\alpha}$,

Casos $B_{3}$ e $C_{3}$ Coordenadas Nulas de Gu-Hu-Inoguchi com parâmetro $\mu=\frac{1}{2} e^{\alpha}$,

Caso $D$ Coordenadas de Tchebyschef II.

Além disso considere $\left\{e_{1}, e_{2}, e_{3}\right\}$ o respectivo referencial móvel juntamente com suas formas de conexão.

Em todos os casos procuramos uma função $\widetilde{\alpha}(u, v)$ tal que phi é o ângulo entre $\widetilde{N}$ e $N$ e

$$
\widetilde{\mathbb{X}}=\mathbb{X}+\lambda r_{\widetilde{\alpha}},
$$

com $\widetilde{N}$ e $r_{\widetilde{\alpha}}$ dados conforme o conforme o esquema abaixo.

\begin{tabular}{|c|c|c|}
\hline Caso & Direção Tangente $r_{\widetilde{\alpha}}$ & Normal $\widetilde{N}$ \\
\hline$B_{1}$ & $\cosh \frac{\widetilde{\alpha}}{2} e_{1}+\sinh \frac{\widetilde{\alpha}}{2} e_{2}$ & $\sinh \phi\left(\sinh \frac{\widetilde{\alpha}}{2} e_{1}+\cosh \frac{\widetilde{\alpha}}{2} e_{2}\right)+\cosh \phi N$ \\
\hline$B_{2}$ & $\exp \frac{-\widetilde{\alpha}-\alpha}{2} e_{1}+\exp \frac{\widetilde{\alpha}-\alpha}{2} e_{2}$ & $\sinh \phi\left(\exp \frac{-\widetilde{\alpha}-\alpha}{2} e_{1}+\exp \frac{\widetilde{\alpha}-\alpha}{2} e_{2}\right)+\cosh \phi N$ \\
\hline$B_{3}$ & $\exp \frac{-\widetilde{\alpha}-\alpha}{2} e_{1}+\exp \frac{\widetilde{\alpha}-\alpha}{2} e_{2}$ & $\sinh \phi\left(\exp \frac{-\widetilde{\alpha}-\alpha}{2} e_{1}-\exp \frac{\widetilde{\alpha}-\alpha}{2} e_{2}\right)+\cosh \phi N$ \\
\hline$C_{1}$ & $\sinh \frac{\widetilde{\alpha}}{2} e_{1}+\cosh \frac{\widetilde{\alpha}}{2} e_{2}$ & $\sin \phi\left(\cosh \frac{\widetilde{\alpha}}{2} e_{1}+\sinh \frac{\widetilde{\alpha}}{2} e_{2}\right)+\cos \phi N$ \\
\hline$C_{2}$ & $\exp \frac{\widetilde{\alpha}-\alpha}{2} e_{1}-\exp \frac{-\widetilde{\alpha}-\alpha}{2} e_{2}$ & $\sin \phi\left(\exp \frac{\widetilde{\alpha}-\alpha}{2} e_{1}+\exp \frac{-\widetilde{\alpha}-\alpha}{2}\right)+\cos \phi N$ \\
\hline$C_{3}$ & $\exp \frac{\widetilde{\alpha}-\alpha}{2} e_{1}-\exp \frac{-\widetilde{\alpha}-\alpha}{2} e_{2}$ & $\sin \phi\left(\exp \frac{\widetilde{\alpha}-\alpha}{2} e_{1}+\exp \frac{-\widetilde{\alpha}-\alpha}{2}\right)+\cos \phi N$ \\
\hline$D$ & $\cos \frac{\widetilde{\alpha}}{2} e_{1}+\sin \frac{\widetilde{\alpha}}{2} e_{2}$ & $\cosh \phi\left(-\sin \frac{\widetilde{\alpha}}{2} e_{1}+\cos \frac{\widetilde{\alpha}}{2} e_{2}\right)+\sinh \phi N$ \\
\hline
\end{tabular}

Perceba que em cada caso, $r_{\widetilde{\alpha}}$ define uma direção no plano tangente a superfície e que nos casos $B_{1}, B_{2}, B_{3}$ e $D$ é uma direção tipo-espaço e nos casos $C_{1}, C_{2}$ e $C_{3}$ uma direção tipo-tempo e que o ângulo $\phi$ é o ângulo congruência. O que está de acordo com a Definição (4.2) de Congruência Pseudo-Esférica.

Diferencie exteriormente $\widetilde{\mathbb{X}}$ e imponha a condição $\langle d \widetilde{\mathbb{X}}, \widetilde{N}\rangle=0$. Utilizando a independência linear entre $d u$ e $d v$ esta condição implicará em um sistema do tipo de Frobenius análogo ao Sistema (4.36). E a condição de integrabilidade de tal sistema será precisamente a EDP Natural da superfície $\mathbb{X}$ em questão, de modo que o Teorema de Frobenius 4.4 nos assegurará a existência da função $\widetilde{\alpha}$ solução deste sistema. Consequentemente as expressões $\widetilde{\mathbb{X}}=\mathbb{X}+\lambda r_{\widetilde{\alpha}}$ definirão uma superfície congruente a $\mathbb{X}$.

Observação 4.2. O caso $D$ do Teorema 4.7 poderia ser enunciado analogamente em termos de uma superfície $\mathbb{X}$ tipo-tempo com curvatura Gaussiana constante igual a $-\frac{\cosh ^{2} \phi}{\lambda^{2}}$. 
Nesta situação, na demonstração deve-se tomar $\mathbb{X}(u, v)$ parametrizada por parâmetros $(u, v)$ de Tchebyschef III e usar como direção de congruência

$$
r_{\widetilde{\alpha}}=\cosh \phi e_{1}+\sinh \phi e_{2},
$$

e normal

$$
\widetilde{N}=\cosh \phi\left(\sinh \frac{\widetilde{\alpha}}{2} e_{1}+\cosh \frac{\widetilde{\alpha}}{2} e_{2}\right)+\sinh \phi N
$$




\section{Capítulo 5}

\section{Conclusões}

Nesta dissertação, baseada nos trabalhos [15, 4], [10, [11, [19] and [12, generalizamos o conceito de congruência pseudo-esférica para superfícies no Espaço-Tempo de Minkowski. Mostramos que existe um análogo do Teorema de Bäcklund para superfícies no Espaço-Tempo de Minkowski e fizemos uma demonstração deste teorema usando o Método do Referencial Móvel.

Esta dissertação não exige do leitor o conhecimento prévio sobre Geometria no Espaço de Minkowski, nem o conceito do Método do Referencial Móvel, pois os capítulos 1 e 2 fornecem o material necessário sobre os temas. No Capítulo 1 discutimos sobre a geometria do Espaço-Tempo de Minkowski, demonstramos algumas de suas propriedades e introduzimos o conceito de Superfícies e sua geometria. No Capítulo 2, introduzimos o conceito de Formas Diferenciais e demonstramos algumas de suas propriedades. A partir disso descrevemos o Método do Referencial Móvel para Superfícies no Espaço de Minkowski.

No Capítulo 3 trouxemos uma das aplicações do Método do Referencial Móvel para Superfícies no Espaço-Tempo de Minkowski. Vimos que é possível classificar todas Superfícies Tipo-Espaço e Tipo-Tempo com curvatura constante não nula via soluções de determinadas Equações Diferenciais Parciais. Isto é, mostramos que existe uma relação unívoca entre existência de soluções das Equações de sin-Gordon, sinh-Gordon, cosh-Gordon, sin-Laplace, sinh-Laplace e Liouville e a existência (e unicidade a menos de um Movimento Rígido no espaço de Minkowski) de superfícies Tipo-Tempo e Tipo-Espaço com curvatura Gaussiana constante não nula.

No Capítulo 4 introduzimos o conceito clássico de congruência pseudo-esférica para o espaço Euclidiano e mostramos como adaptá-lo para o Espaço-Tempo de Minkowski. Demonstramos o clássico Teorema de Bäcklund, que nos dá uma condição necessária para a 
ocorrência de uma Congruência pseudo-esférica entre superfícies no espaço Euclidiano. A condição necessária é que as duas superfícies devem ter a mesma curvatura Gaussiana e este valor comum deve ser uma constante negativa.

Mostramos que existe uma versão do Teorema de Bäcklund para superfícies no Espaço de Minkowski, qual nos garante que uma condição necessária para que exista uma congruência pseudo-esférica entre duas superfícies. A condição necessária é que as duas superfícies devem ter a mesma curvatura Gaussiana constante positiva ou negativa.

Buscamos condições suficientes para a ocorrência de congruência pseudo-esféricas entre superfícies no Espaço-Tempo de Minkowski. Mostramos que existem resultados análogos no Espaço-Tempo de Minkowski do Teorema clássico de Integrabilidade. Isto é, mostramos que existem condições suficientes para existência de congruência pseudo-esférica entre superfícies no Espaço-Tempo de Minkowski.

Partindo da classificação das superfícies dadas no Capítulo 3, juntamente com os resultados do Capítulo 4, mostramos que é possível construir uma família a dois parâmetros de soluções das Equações de sin-Gordon, sinh-Gordon, cosh-Gordon, sin-Laplace, sinh-Laplace e Liouville a partir de uma solução inicial. 


\section{Referências Bibliográficas}

[1] S. C. de Almeida e F. A. A. Pimentel, O Teorema de Bäcklund segundo S. S. Chern Matemática Universitária, número 20/21, 23-45, junho/dezembro 1996.

[2] P. V. Araújo, Geometria Diferencial, Segunda edição, Coleção Matemática Universitária, editora do IMPA, 2008.

[3] S.S. Chern, W.H. Chen e K.S. Lam, Lectures on Differential Geometry, Series on University Mathematics Vol. I, World Scientific Publishy, First Published 1999, Reprinted 2000 .

[4] S.S. Chern, Geometrical interpretation of the sinh-Gordon equation, Annales Polonici Mathematici XXXIX, 1981.

[5] M.P. do Carmo, Geometria Diferencial de Curvas e Superfícies, Coleção de Textos Universitários SBM, quinta edição, Rio de Janeiro, 2005.

[6] M.P. do Carmo, Differential Forms and Applications, Universitext, Springer 1st ed. 1994. Corr. 2nd printing, ISBN-10: 3540576185, 1998.

[7] M.P. do Carmo, Geometria Riemanniana, Projeto Euclides, Rio de Janeiro: Instituto de Matemática Pura e Aplicada, segunda edição, ISBN 85-244-0036-6, 1988.

[8] D. G. de Figueiredo, Equações Diferenciais e Aplicações, $12^{\circ}$ Colóquio Brasileiro de Matemática, IMPA, 1979.

[9] L.P. Eisenhart, Minimal surfaces in Euclidean four-space, Am. Math. Soc. 215-236, 1911.

[10] Hu Hesheng, The constrution of hyperbolic surfaces in 3 dimensional Minkowski space and sinh-Laplace equation, Acta Mathematica Sinica, New Series, Vol. 1, No.1, pp. 79-86, 1984. 
[11] C. H. Gu, H. Hu and Z. Zhou, Darboux Transformations in Integrable Systems: Theory and their Applications to Geometry, Mathematical Physics Studies 26, Published by Springer, 2005.

[12] C.H. Gu, H.S. Hu, J. Inoguchi, On time-like surfaces of positive constant gaussian curvature and imaginary principals curvatures, Elsevier, Journal of Geometry and Physics, 41, 296-311, 2002.

[13] H. P. Bueno, Algebra Linear: Um segundo curso Coleção Textos Universitários, editora da Sociedade Brasileira de Matemática, 2006.

[14] T. A. Ivey and J. M. Landsberg, Cartan for Beginners: Differential Geometry via Moving Frames and Exterior Differential Systems, Graduate Studies in Mathematics, Volume 61, American Mathematical Society, 2003.

[15] L. V. McNertney, One-parameter families of surfaces with constant curvature in Lorentz 3-space, Ph. D. thesis, Brown University, 1980.

[16] R. López, Differential Geometry of curves and surfaces in Lorentz-Minkowski space, International Eletronic Journal of Geometry, Volume 7 No. 1 44-107, 2014.

[17] B.O'Neill, Semi-Riemannian geometry with applications to relativity, Series: Pure and Applied Mathematics, Academic Press Inc, 1983.

[18] B.O'Neill, Elemetary Differential Geometry, Series: Pure and Applied Mathematics, Academic Press Inc., 1966.

[19] B. Palmer, Bäcklund transformations for surfaces in Minkowski space, J. Math. Phys. 31 (12), December 1990.

[20] K. Tenenblat, Introdução à Geometria Diferencial, Segunda Edição. Editora Edgard Blücher Ltda, 2008.

[21] C. Tian, Bäcklund transformation on surfaces with $K=-1$ in $\mathbb{R}^{2,1}$, Journal of Geometry and Physics, volume 22, 212-218, 1997. 


\section{Índice Remissivo}

Ângulo

da congruência pseudo-esférica, 101

hiperbólico, 20

Aplicação

auto-adjunta, 28

Normal de Gauss, 25

Base ortogonal, 9

Caráter causal

de uma superfície, 23

de um plano, 15

de um subespaço, 11

de um vetor, 10

de uma reta, 15

Co-Referencial Móvel

adaptado a uma superfície em $\mathbb{E}^{3}, 47$

adaptado a uma superfície em $\mathbb{L}^{3}, 55$

Nulo, 63

Cone tipo-tempo de um vetor, 16

Congruência

pseudo-esférica em $\mathbb{E}^{3}, 95$

pseudo-esférica em $\mathbb{L}^{3}, 99$

pseudo-esférica tipo-espaço, 100

pseudo-esférica tipo-tempo, 100

pseudo-esférica de retas em $\mathbb{L}^{3}$, 101

Coordenadas

de Tchebyschef em $\mathbb{E}^{3}, 74$ de Tchebyschef I, 80

de Tchebyschef II, 81

de Tchebyschef III, 82

de Tchebyschef IV, 83

Nulas de Gu-Hu-Inoguchi, 90

Nulas de McNertney, 88

Curvatura

(s) principais, 29

Gaussiana, 27

Média, 27

Desigualdade

de Cauchy-Schwarz, 17

reversa de Cauchy-Schwarz, 17

Diferencial Exterior, 44

Distância

da congruência pseudo-esférica, 101

EDP Natural, 68

Equação

de cosh-Gordon, 91, 95, 116

de Liouville, 69, 89, 95, 116

de sine-Gordon, $74,80,94,95,116$

de sine-Gordon Negativa, 95

de sine-Laplace, 83, 95, 116

de sinh-Gordon, 82

de sinh-Gordon Negativa, 69, 84, 95, 116

de sinh-Laplace, 95, 116

de sinh-Laplace Negativa, 77 
Equações de estrutura

de um referencial móvel em $\mathbb{E}^{3}, 50$

de um referencial móvel em $\mathbb{L}^{3}, 59$

Espaço de De Sitter, 17

Espaço dual, 35

Forma

indefinida, 7

não-degenerada, 7

negativa definida, 7

positiva definida, 7

bilinear, 5

bilinear simétrica, 5

quadrática, 7

Formas de conexão

de um co-referencial em $\mathbb{E}^{3}, 47$

de um co-referencial em $\mathbb{L}^{3}, 55$

Formas Diferenciais

0-forma diferencial, 42

1-forma diferencial, 37

2-forma diferencial, 41

terna de 0-formas diferenciais, 43

terna de 1-formas diferenciais, 43

terna de 2-formas diferenciais, 44

Lema de Cartan, 51, 59

Métrica

Euclidiana, 10

Lorentziana, 10

Minkowisk

espaço de, 10

Norma

de um vetor em $\mathbb{L}^{3}, 16$
Operador

de Weingarten, 26

Forma, 26

Plano

Tangente, 22

Degenerado, 15

Lorentziano, 15

Riemanniano, 15

Plano Hiperbólico, 17

Ponto

umbílico, 30

Produto

Interno, 9

Exterior, 39, 45

Tensorial, 38

Vetorial Lorentziano, 18

Referencial Móvel

adaptado a uma superfície em $\mathbb{E}^{3}, 46$

adaptado a uma superfície em $\mathbb{L}^{3}, 54$

de Tchebyschef em $\mathbb{E}^{3}, 74$

de Tchebyschef I, 80

de Tchebyschef II, 81

de Tchebyschef III, 82

de Tchebyschef IV, 84

Nulo, 63

Nulo de Gu-Hu-Inoguchi, 90

Nulo de McNertney, 88

Segunda forma fundamental, 27

Subespaço

tipo-espaço, 11

tipo-luz, 11

tipo-tempo, 11 
Superfície

não-degenerada, 23

parametrizada em $\mathbb{L}^{3}, 22$

regular em $\mathbb{L}^{3}, 22$

simples em $\mathbb{L}^{3}, 22$

tipo-espaço, 23

tipo-tempo, 23

umbílica, 30

Weingarten-diagonalizável, 29

Superfície tipo-luz, 23

Teorema

de Bäcklund em $\mathbb{E}^{3}, 96$

de Bäcklund em $\mathbb{L}^{3}$, 105

de Frobenius, 108

de Picard, 108

Vetor

(es), ângulo entre, 20

cone tipo tempo de um, 16

sinal de um, 54

Tangente, 22

norma de um, 16

tipo-espaço, 10

tipo-luz, 10

tipo-tempo, 10

unitário, 16

Weingarten-diagonalizável

superfície, 29 